\title{
Laterally Grown ZnO Nanowires for Sensing Applications
}

\author{
by \\ Campbell Stuart Baldwin Matthews
}

\author{
A thesis \\ submitted to the Victoria University of Wellington \\ in fulfilment of the \\ requirements for the degree of \\ Master of Science \\ in Physics.
}

Victoria University of Wellington

2018 



\begin{abstract}
Zinc oxide nanowires are a semiconducting material that has many uses in electronic applications. In particular, $\mathrm{ZnO}$ nanowires have been used in field effect transistors and applied as sensors for the detection of gases, biomolecules, UV light and as pressure sensors. $\mathrm{ZnO}$ nanowires can be fabricated using many different methods, but most require the use of high tempertures and have extensive setup costs. Hydrothermal growth, however, provides a cheap and low temperture method for growing $\mathrm{ZnO}$ nanowires. Much work has been done on the synthesis and charcetristaion of $\mathrm{ZnO}$ nanowires grown using hydrothermal growth, in partiuclar for photovoltaic applications. Little work has been done on the performace of hydrothermally grown $\mathrm{ZnO}$ nanowires in field effect transtors. This thesis looks at applying hydrothermally grown $\mathrm{ZnO}$ nanowires as field effect transistors (FET). The FETs are characterised and developed with the intention of using them in senseing applications.

The nanowire FET structure is optimised for sensing by developing a method that constrains the nanowires to exclusively lateral growth. A Ti capping layer is fabricated on top of a $\mathrm{ZnO}$ seed layer. The $\mathrm{ZnO}$ seed layer is then etched with dilute acid so that the $\mathrm{Ti}$ layer overhangs the $\mathrm{ZnO}$. This acts as a physical barrier to vertical wire growth from the $\mathrm{ZnO}$ seed layer. The maximum deviation of the nanowires from the horizontal can be controlled by etching for different times.

Two device types are fabricated using different size nanowires. One uses large nanowires, or nanorods (diameter $400 \mathrm{~nm}$ ), while the second device type uses a hybrid structure of large nanorods with much thinner nanowires (diameter 20 $\mathrm{nm}$ ) growing off them. Both device types are characterised as FETs in dry conditions and also when immersed in a number of different liquids. Two different gating setups are also used with the $\mathrm{Si} / \mathrm{SiO}_{2}$ substrate used as a backgate and a $\mathrm{Ag} / \mathrm{AgCl}$ electrode inserted into liquid as a topgate.
\end{abstract}


The large nanorods only show field dependence when wet due to the large capacitance of the elctric double layer and enhanced band bending. The wet nanorods can achieve on/off ratios of $10^{3}$. In contrast, the thinner nanowires show field dependence both when dry and when wet. On/off ratios of more than $10^{4}$ are achieved. In general the nanowires have superior on off ratios and smaller off current due to their larger surface to volume ratio.

Attempts are made to functionalise the nanowires with aptamers so that they can be used as a biosensor. The functionalisation procedure is documented, however the overall procedure proves to be unsuccessful due to the instability and dissolution of the nanowires in tris buffer. The rate of decay in buffer solution is investigated.

Both device types are also tested as gas sensors for humidity and ethanol detection. The nanorods show no apparent detection, while the nanowires show some response to ethanol. Further development of the experimental setup is necessary to better characterise the devices.

Finally future work on these nanowires is discussed and possible improvements proposed for future development as biosensors and gas sensors. 
"Remember kids, the only difference between screwing around and science is writing it down"

- Adam Savage 


\section{Acknowledgements}

I would first like to acknowledge my supervisor, Natalie Plank, and express my appreciation for all the advice, guidance and feedback she has provided during this research. Thank you for the support you have given me.

I should also mention the help, training and discussion provided by members of the Nanomaterials Devices Group including Conor Burke-Govey, Leo Browning, Mohsen Maddah and Murugathas Thanihaichelvan (Selvan). Thanks are also extended to the members of the Rare Earth Nitride Group and others who work in the cleanroom or attend the weekly group meeting, including: Will HolmesHewett, Felicia Ullstad, Jackson Miller, Ali Shaib, Jay Chan, Hamish Colenso, Matt Cryer, Ben Ruck, Franck Natali and Joe Trodahl.

I am grateful to my parents, Heather and Martin, who have provided me with support, encouragement and food during my studies, thank you. I also acknowledge my brothers; Lincoln, for banging his gavel, and Binney, who kept out of the way. I would like to thank the rest of my family, and all other members of the Imperial Household of Mongravia, including the Mighty Zorgon of the East, Slayer of the Vargen Dragon and Doer of Wondrous Deeds, for the encouragement and interest they have shown in my studies.

I am also thankful to Vinesh Patel, from the Microsoft Support Office, who called me to say that my computer had been infected with a virus. Without him this thesis could not have been finished.

Finally, for anyone else who thinks that they should have been mentioned, please feel free to add you name at the bottom of this page. 


\section{Contents}

1 Introduction 1

2 Zinc Oxide and Nanowire Growth 5

2.1 Zinc Oxide ......................... 5

2.2 Hydrothermal Growth . . . . . . . . . . . . . . . 7

2.2.1 Chemical Reactions . . . . . . . . . . . . . . . 7

2.2 .2 Crystal Nucleation . . . . . . . . . . . . . . . . 99

2.2.3 Crystal Shape and Polyethyleneimine . . . . . . . . . . 9 9

2.3 Summary ............................ 11

$\begin{array}{lll}\text { ZnO Nanowire Devices and Sensors } & 13\end{array}$

3.1 Field Effect transistors . . . . . . . . . . . . . . . . . . 13

3.1 .1 Band Bending . . . . . . . . . . . . . . 14

3.1 .2 Liquid Gating . . . . . . . . . . . . . . . . 14

3.1.3 FET Measurements and Parameters . . . . . . . . . . 16

$3.2 \mathrm{ZnO}$ Nanowire Devices . . . . . . . . . . . . . . . . . . . . 17

3.2.1 Field Effect Transistors . . . . . . . . . . . . . . . . . . 17

3.2 .2 Gas Sensors . . . . . . . . . . . . . . . . . . . . 19

3.2.3 Photo Sensors . . . . . . . . . . . . . . . 20

3.2.4 Hysteresis in ZnO Nanowire FETs . . . . . . . . . . . . . 21

3.3 Biofuncionalsiation . . . . . . . . . . . . . . . 21

3.3 .1 Aptamers ........................ 21

3.3.2 ZnO Biosensors .................... 22

3.4 Summary . . . . . . . . . . . . . . . . . . 24

4 ZnO Nanowire Device Fabrication and Characterisation 25

$4.1 \mathrm{ZnO}$ Nanowire Device Fabrication . . . . . . . . . . . . . . 25 
4.1 .1 Substrate Preparation . . . . . . . . . . . . . 25

4.1 .2 Photolithography .................. 26

4.1 .3 Sputtering ..................... 26

4.1 .4 Acid Etch . . . . . . . . . . . . . . . . . 28

4.1 .5 Hydrothermal Growth . . . . . . . . . . . . . . 28

4.1.6 Scanning Electron Microscope . . . . . . . . . . . . . . . 28

4.1 .7 Encapsulation . . . . . . . . . . . . . . . . . 29

4.2 Aptamer Functionalisation . . . . . . . . . . . . . . . 30

4.3 Electrical Measurements . . . . . . . . . . . . . . . . . 32

4.3 .1 Backgate Setup . . . . . . . . . . . . . 32

4.3.2 Liquid and Topgate Setup . . . . . . . . . . . . 32

4.4 Gas Rig . . . . . . . . . . . . . . . . . . . 33

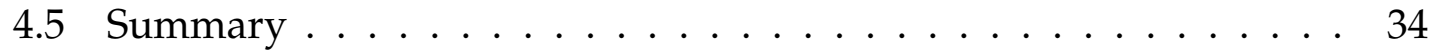

5 ZnO Nanowire Structure and Morphology 35

5.1 Lateral Wire Growth . . . . . . . . . . . . . . . . . . 35

5.1 .1 Acid Etch of ZnO Seed Layer . . . . . . . . . . . . . . 37

5.1 .2 Wire morphology . . . . . . . . . . . . . . 39

5.2 ZnO Nanorod and Nanowire Device Structures . . . . . . . . . . . . 42

5.2.1 Nanorod Devices . . . . . . . . . . . . . . . . . . 42

5.2 .2 Nanowire Devices . . . . . . . . . . . . . . . 43

5.3 Conclusions . . . . . . . . . . . . . . . 45

6 Electrical Characterisation of ZnO Nanorod and Nanowire Devices $\quad 47$

6.1 Nanorod Electrical Characterisation . . . . . . . . . . . . . 47

6.1 .1 Dry ......................... 47

6.1 .2 Liquid Gated (DI Water) . . . . . . . . . . . . . . 47

6.1.3 Hardbaking SU-8 and Nanorods . . . . . . . . . . . . . . 51

6.2 Nanowire Electrical Characterisation . . . . . . . . . . . . 52

6.2 .1 Dry ............................ 52

6.2 .2 Liquid Gated (DI Water) . . . . . . . . . . . . . . . 54

6.2 .3 Liquid Gated (Tris Buffer) . . . . . . . . . . . . . 56

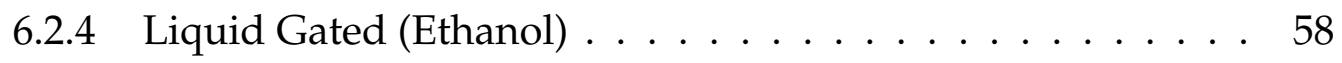


6.3 Hysteresis in $\mathrm{ZnO}$ Nanorods and Nanowires . . . . . . . . . . . 59

6.4 Conclusions ............................ 62

7 Functionalisation Results $\quad 63$

7.1 Surface Modification . . . . . . . . . . . . . . . . . 63

7.1 .1 Oxygen Plasma . . . . . . . . . . . . . . . 63

7.1.2 GOPS Functionalisation . . . . . . . . . . . . . 64

7.2 Aptamer Functionalisation . . . . . . . . . . . . . 66

7.3 Discussion ............................ 68

7.4 Conclusion . . . . . . . . . . . . . . . . 69

8 Gas Sensing $\quad 71$

8.1 Humidity Sensing . . . . . . . . . . . . . . . . 71

8.1 .1 Nanorods . . . . . . . . . . . . . . 71

8.1 .2 Nanowires . . . . . . . . . . . . . . 72

8.1 .3 Discussion ....................... 73

8.2 Ethanol Sensing . . . . . . . . . . . . . . . 74

8.2.1 Nanorods ..................... 74

8.2 .2 Nanowires .................... 74

8.2 .3 Discussion ................... 76

8.3 Conclusion . . . . . . . . . . . . . . . 77

9 Conclusions $\quad 79$

9.1 Summary . . . . . . . . . . . . . . . . . 79

9.2 Future Work . . . . . . . . . . . . . . . . . . 80

9.2.1 Biosensors ...................... 80

9.2 .2 Gas Sensors . . . . . . . . . . . . . . . . 82

9.3 Final Word . . . . . . . . . . . . . . . . . . . . . . 83

A Reproducibility of Devices $\quad 85$

$\begin{array}{ll}\text { List of Abbreviations } & 87\end{array}$

$\begin{array}{lr}\text { References } & 89\end{array}$ 
CONTENTS 


\section{Chapter 1}

\section{Introduction}

The first use of $\mathrm{ZnO}$ in electronics was in the 1920s as a cat-whisker detector for radio sets [1]. Since then research on $\mathrm{ZnO}$ continued with papers published in 1935 on the lattice parameters of $\mathrm{ZnO}$ [2], its electrical and optical properties in 1954 [3], and its piezoelectronic properties in 1960 [4]. More recently there has been an increase in the interest in $\mathrm{ZnO}$. This has stemmed from the advancement of nanoscale fabrication, investigations into $p$ type doping, thin film field effect transistors and the possible use of $\mathrm{ZnO}$ as a transparent semiconductor in optoelectronics [5]. Recent reviews of $\mathrm{ZnO}$ and its applications in devices have been published by Özgür et al. [6,7].

$\mathrm{ZnO}$ is a natively $\mathrm{n}$ type semiconductor and in addition, $\mathrm{ZnO}$ has a wide band gap, large exciton binding energy, large cohesive energy and is transparent in the visible spectrum [6-8]. These properties make $\mathrm{ZnO}$ a suitable material for high temperature semiconductor applications or transparent electronics that silicon is not suited for [6-10]. Furthermore $\mathrm{ZnO}$ can be readily fabricated into an extremely large array of different nanostructures include nano- belts, cages, combs, helices, propellers, rings, springs and wires [11], which makes $\mathrm{ZnO}$ an exciting option for nanoscale semiconducting electronics.

A developing area is the use of $\mathrm{ZnO}$ in field effect transistors (FETs) [6,7]. Transistors are effectively electronic switches that operate by controlling the current flow between two terminals with a voltage applied to the third terminal [12]. $\mathrm{ZnO}$ nanowires in particular show promise. Their large surface to volume ratio is advantageous in FETs, as the fundamental operation of FETs relies on band bending at the surface of the semiconductor [12]. The large surface to volume ratio is also useful in sensing applications.

Sensors have been fabricated using many different semiconductors including carbon nanotubes $[13,14]$, silicon nanowires $[14,15]$, conducting polymer nanowires [14], $\mathrm{ZnO}$ thin films [16] and $\mathrm{ZnO}$ nanowires [16]. These sensors operate by measuring the change in drain-source current caused by changes in the surface potential due to the binding or reconfiguration of molecules on the surface of the semiconductor [13-17]. This includes direct adsorption of chemical species or the reformation of molecules functionalised onto the surface of the semiconductor. $\mathrm{ZnO}$ nanowire chemiresistor sensors have been designed to measure the concentration of chemical vapours [7] and $\mathrm{ZnO}$ nanowire FETs have also been chemically functionalised to detect biological molecules [16]. These biosensors often make use of aptamers [18], short strands of DNA or RNA, bound to the surface of the $\mathrm{ZnO}$ and can achieve extremely high selectivity [19]. 
There is a large variety of possible synthesis techniques for $\mathrm{ZnO}$ nanowires including thermal evaporation [20], pulsed laser deposition [21], metalorganic vapour phase epitaxy [22], chemical vapour deposition [23], chemical vapour transport and condensation [24], and vapour-liquid-solid method [19]. All of these methods require high temperatures and/or expensive setup costs. In contrast hydrothermal growth is a wet chemistry method that does not have these disadvantages. It uses cheap and readily available precursors and all reactions occur at low temperatures (below $100^{\circ} \mathrm{C}$ ). There has been extensive characterisation of hydrothermally grown $\mathrm{ZnO}$ nanowires [25-35] and many examples of their incorporation into solar cells [33-39]. However, there has been limited study on the use of hydrothermally grown nanowires in FETs and applications as sensors.

The intention of this thesis is to fabricate and characterise hydrothermally grown $\mathrm{ZnO}$ nanowires as FETs with the explicit motivation of developing them into sensors.

Chapter 2 introduces the general background of zinc oxide and the hydrothermal growth of $\mathrm{ZnO}$ nanowires. Interesting chemical, electronic and semiconducting properties of $\mathrm{ZnO}$ are briefly described. The crystal structure of $\mathrm{ZnO}$ is also covered and is pertinent to the hydrothermal growth of $\mathrm{ZnO}$ nanowires, the chemistry of which is then outlined. The mechanics of the crystallisation of $\mathrm{ZnO}$ is discussed and linked to the formation of crystals on $\mathrm{ZnO}$ seeds. The driving force behind the formation of nanowires and how nanowire growth can be promoted, in particular with the growth additive polyethyleneimine, is then discussed.

Chapter 3 covers the operation and theory of field effect transistors (FETs), current uses of $\mathrm{ZnO}$ nanowire devices (in particular FETs) and the biofunctionalisation of $\mathrm{ZnO}$ nanowires. A simple description of the FET structure is given and the field induced band bending in a metal-insulator-semiconductor interface is described. The use of liquid dielectrics in FETs and the formation of an electric double layer is also covered. A summary of some FET parameters that describe the performance of an FET are included. The current state of $\mathrm{ZnO}$ nanowire FETs in literature, with examples, is given. The interaction between $\mathrm{ZnO}$ and chemical species is then discussed and linked to the use of $\mathrm{ZnO}$ nanowire FET gas sensors, UV sensors and the origin of hysteresis in $\mathrm{ZnO}$ nanowire devices. Lastly, the process of biofunctionalisation of $\mathrm{ZnO}$ nanowires, with a focus on aptamers, is described along with the current use of $\mathrm{ZnO}$ nanowire FETs as biosensors.

Chapter 4 provides the experimental techniques and equipment used throughout this thesis, including the processes used to fabricate devices and the electrical measurement setups. All topics are covered in adequate detail such that the devices and measurements made in this thesis could be reproduced.

Chapter 5 deals with the process of optimising the structure of the $\mathrm{ZnO}$ nanowire devices for sensing. Results of investigation into the growth of exclusively lateral $\mathrm{ZnO}$ nanowires are presented. The two finalised device structures used in this 
thesis are then described and referred to as "nanorods" (which use large $\mathrm{ZnO}$ nanowires of about $400 \mathrm{~nm}$ in diameter) and "nanowires" (which make use of smaller $\mathrm{ZnO}$ nanowires of about $20 \mathrm{~nm}$ in diameter).

Chapter 6 presents the results of the electrical characterisation of the nanorod and nanowire devices as FETs. Measurements are made of the devices in both dry and wet environments and multiple gating setups are used, including backgating through the $\mathrm{SiO}_{2}$ and topgating, using a $\mathrm{Ag} / \mathrm{AgCl}$ electrode immersed into liquid. Both the nanowires and nanorods show good switching characteristics when in liquid, but only the nanowires show field dependence when dry. These results are used to select the best device type for functionalisation as a biosensor.

Chapter 7 shows the results of attempts to functionalise the nanowire devices with aptamers for use as a biosensor. This is unsuccessful due to the chemical instability of the nanowires and the rate of decay is explored.

Chapter 8 looks at the use of the devices as gas sensors for ethanol and humidity (water vapour) detection. Only a weak response from the nanowires is achieved. Further development of the gas rig may improve the quality of the results.

Finally chapter 9 provides a summary of conclusions and proposals for future work. 


\section{Chapter 2}

\section{Zinc Oxide and Nanowire Growth}

This chapter details the background of $\mathrm{ZnO}$ and the formation of $\mathrm{ZnO}$ nanostructures. It covers why $\mathrm{ZnO}$ is an interesting material and how it can be manufactured into nanomaterials with novel properties. It details hydrothermal growth as a manufacture method, which is used in this thesis.

\subsection{Zinc Oxide}

$\mathrm{ZnO}$ is a II-VI compound semiconductor. It is natively n-type as a result of the presence of electron donors, usually attributed to oxygen vacancies or interstitial $\mathrm{Zn}$ ions, but the exact origin is still debated $[8,40]$. Further n-type doping can be achieved by doping with group III elements (Al, Ga or In) or VII elements $(\mathrm{Cl}$ or I) [6]. p-type doping is extremely difficult to achieve [41] and doping with group I elements ( $\mathrm{Li}, \mathrm{Na}$ or $\mathrm{K}$ ) or group V elements (N, P or As) is typically unsuccessful. The small ionic radii of the group I and $\mathrm{V}$ elements means that they typically fill interstitial sites and native defects ( $\mathrm{Zn}$ interstitials and oxygen vacancies) often compensate for any effect from these acceptors $[6,41]$. The inability to produce $p-$ type $\mathrm{ZnO}$ remains the main obstacle to the realisation of bipolar $\mathrm{ZnO}$ devices [7].

The bandgap of $\mathrm{ZnO}$ is particularly large at $3.37 \mathrm{eV}$ and has a very large exciton binding energy of $60 \mathrm{meV}$ [6]. Transitions across the band gap correspond to ultra violet radiation (photon wavelength $=368 \mathrm{~nm}$ ) and the high exciton binding energy means that excitons are stable above room temperature. This makes $\mathrm{ZnO}$ a viable material for uses in high temperature UV lasers based on exciton transitions [6-8]. The large band gap of $\mathrm{ZnO}$ also means that it is transparent in the visible spectrum. When manufactured with high doping levels, $\mathrm{ZnO}$ is an attractive material as a transparent electrode in flat panel displays, LEDs and solar cells $[6,7]$.

$\mathrm{ZnO}$ can form in three crystal structures [6, 8, 42]; wurtzite (hexagonal close packed), zinc-blende (face centred cubic) and rock salt (simple cubic). At ambient conditions the natural crystal structure of $\mathrm{ZnO}$ is the wurtzite structure $[6,8,42]$, while the zinc-blende structure only occurs when carefully grown on cubic substrates and the rock salt structure is only possible at high pressures [6]. A diagram 
of the wurtzite structure is shown in figure 2.1. Each $\mathrm{Zn}^{2+}$ ion is bound to four $\mathrm{O}^{2-}$ in a tetrahedral arrangement and vice versa. The structure can be described by the lattice vectors $\mathbf{a}, \mathbf{b}$ and $\mathbf{c}$ [43]. The associated lattice constants are $\mathbf{a}=\mathbf{b}=$ $3.25 \pm 0.02 \AA$ and $c=5.21 \pm 0.02 \AA[6]$.

The wurtzite structure can also be described using a four index miller index system ( $h k i l)$ (note that $h+k=-i$ ) [43]. A diagram of some low index planes is shown in figure 2.2. The structure can be described as alternating layers of $\mathrm{Zn}^{2+}$ and $\mathrm{O}^{2-}$ ions along the crystal's c axis. This means that the top (0001) plane will consist of exclusively $\mathrm{Zn}^{2+}$, while the bottom (0001) plane will consist of $\mathrm{O}^{2-}$ ions. The lateral faces will then consist of interchanging $\mathrm{Zn}^{2+}$ and $\mathrm{O}^{2-}$ ions. The overall result is that the lateral faces will be effectively neutral, while the top/bottom faces will be slightly polar. The polarity of the different faces is important during hydrothermal growth and is fundamental to the use of growth additives that influence crystal shape (see section 2.2.3).

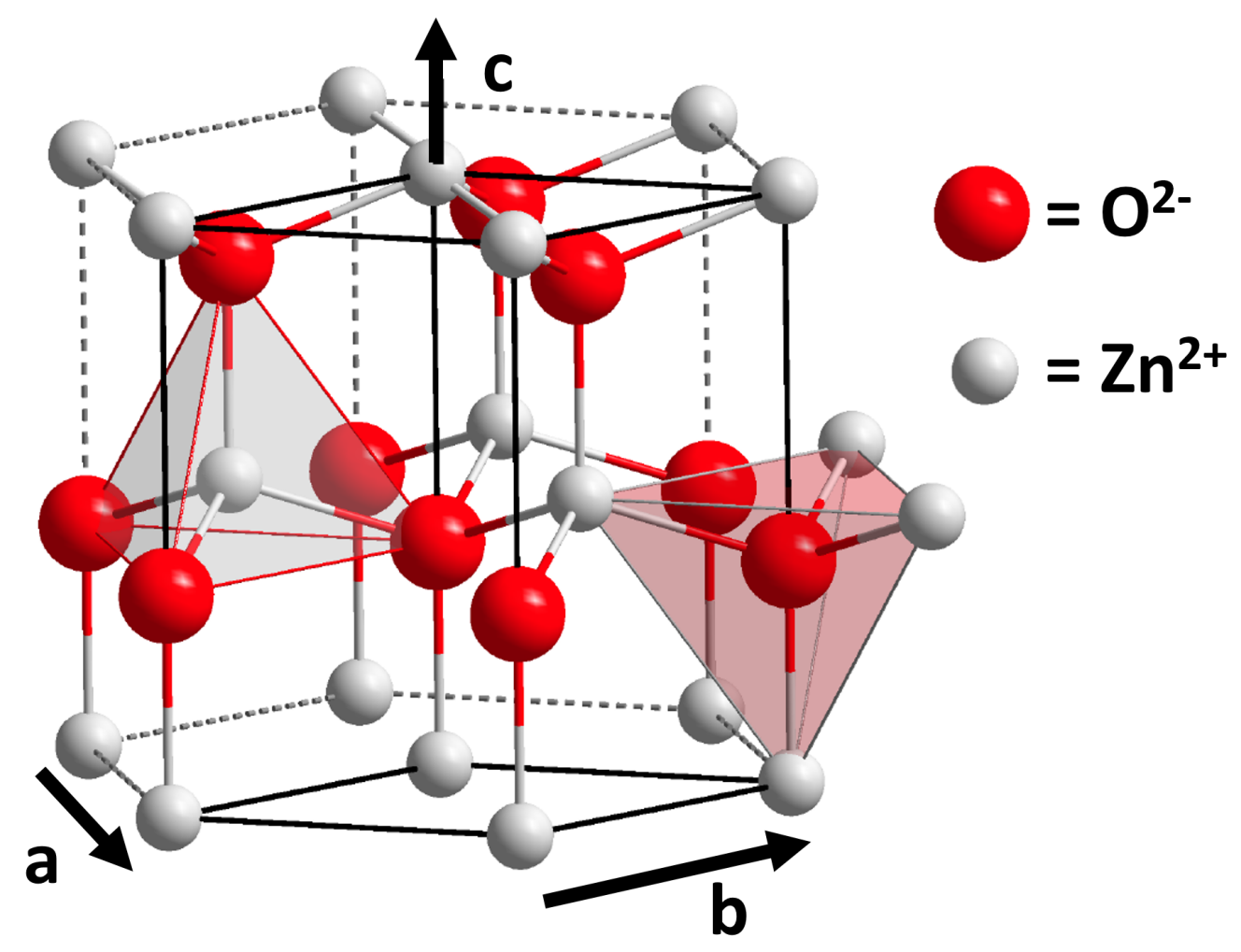

Figure 2.1: The wurtzite crystal structure of $\mathrm{ZnO}$. It is a hexagonal structure formed from tetrahedrons of $\mathrm{Zn}^{2+} / \mathrm{O}^{2-}$ ions. The lattice vectors $\mathbf{a}, \mathbf{b}$ and $\mathbf{c}$ are labelled. Figure modified from [44]. 


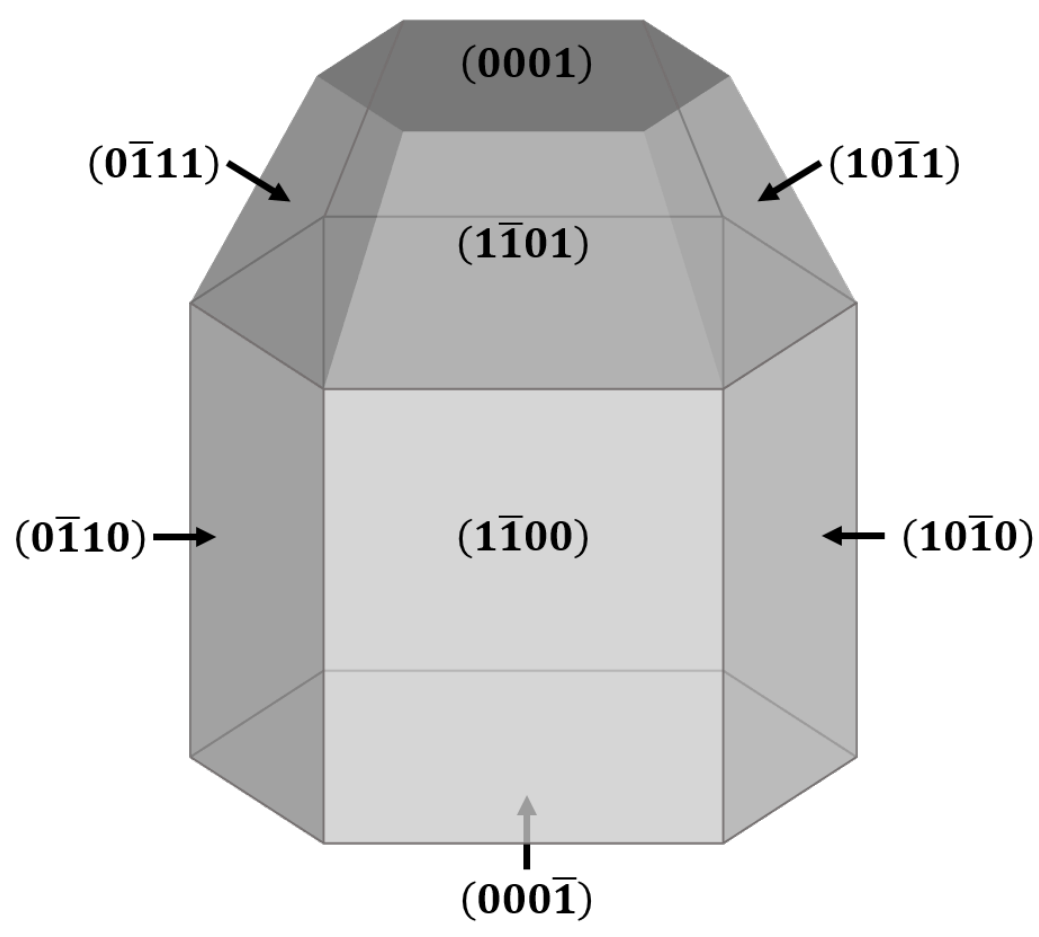

Figure 2.2: A diagram showing low index planes of a hexagonal (e.g. Wurtzite) crystal.

\subsection{Hydrothermal Growth}

A number of different techniques have been developed to synthesise high quality $\mathrm{ZnO}$ nanowires, including thermal evaporation [20], pulsed laser deposition [21], metalorganic vapour phase epitaxy [22], chemical vapour deposition [23], chemical vapour transport and condensation [24], and vapour-liquid-solid method [19]. These methods, however, require high temperatures (some hundreds of degrees celsius) and/or high vacuums (as low as $10^{-6}$ Torr).

An alternative method is hydrothermal growth, which has the major advantage of circumventing the large energetic and material investments required for the aforementioned methods. Hydrothermal growth is a synthesis technique that makes use of an aqueous salt solution to form $\mathrm{ZnO}$ crystals. Growth temperatures do not exceed $100^{\circ} \mathrm{C}$ and the chemical precursors are cheap and readily available.

\subsubsection{Chemical Reactions}

The exact chemical precursors used in hydrothermal growth may vary depending on the intended crystal shape $[45,46]$, but in general the key reactants for $\mathrm{ZnO}$ nanowires are zinc nitrate, $\mathrm{Zn}\left(\mathrm{NO}_{3}\right)_{2}$, (which acts as a source of $\mathrm{Zn}^{2+}$ ions) and some amine containing compound, typically hexamethylenetetramine, 
$\left(\mathrm{CH}_{2}\right)_{6} \mathrm{~N}_{4}$, (also known as methenamine or HMT, a hydrocarbon cage structure containing tertiary amines). All chemical reactions occur in aqueous solution, with the initial reaction being the decomposition of zinc nitrate [29-31,47]:

$$
\mathrm{Zn}\left(\mathrm{NO}_{3}\right)_{2} \rightarrow \mathrm{Zn}^{2+}+2 \mathrm{NO}_{3}^{-}
$$

The next steps of the reaction, however, are not well established and there are differing opinions on the exact reactions. A common postulate is that the amine source, in this case HMT, also decomposes to form ammonia and formaldehyde [29-31, 47].

$$
\left(\mathrm{CH}_{2}\right)_{6} \mathrm{~N}_{4}+6 \mathrm{H}_{2} \mathrm{O} \rightarrow 4 \mathrm{NH}_{3}+6 \mathrm{CH}_{2} \mathrm{O}
$$

It is then stipulated that the ammonia reacts with water to form hydroxide, which in turn reacts with $\mathrm{Zn}^{2+}$ to form $\mathrm{ZnO}$

$$
\begin{gathered}
\mathrm{NH}_{3}+\mathrm{H}_{2} \mathrm{O} \rightarrow \mathrm{NH}_{4}^{+}+\mathrm{OH}^{-} \\
\mathrm{Zn}^{2+}+2 \mathrm{OH}^{-} \rightarrow \mathrm{ZnO}+\mathrm{H}_{2} \mathrm{O}
\end{gathered}
$$

The alternative view is that $\mathrm{Zn}^{2+}$ reacts directly with water, possible via a hydroxide complex:

$$
\begin{gathered}
\mathrm{Zn}^{2+}+\mathrm{H}_{2} \mathrm{O} \rightarrow \mathrm{ZnO}+2 \mathrm{H}^{+} \\
\mathrm{Zn}^{2+}+2 \mathrm{H}_{2} \mathrm{O} \rightleftharpoons \mathrm{Zn}(\mathrm{OH})_{2}+2 \mathrm{H}^{+} \rightleftharpoons \mathrm{ZnO}+\mathrm{H}_{2} \mathrm{O}+2 \mathrm{H}^{+}
\end{gathered}
$$

These reactions are supported by a number of experiments [30,31] which have shown that the decomposition of HMT (equation 2.2) is quite slow and effectively negligible over typical growth times (some hours). Therefore, the formation of $\mathrm{ZnO}$ is independent of HMT decomposition and it is unlikely that HMT reacts as described in equations 2.2-2.4. Analysis of the $\mathrm{pH}$ dependant concentration of the different chemical species in hydrothermal growth suggests that HMT may act as a $\mathrm{pH}$ buffer [30]. This manipulates the $\mathrm{pH}$ of the solution such that the direct formation of $\mathrm{ZnO}$ from $\mathrm{Zn}^{2+}$ (equation 2.5) is favoured. The indirect route (equation 2.6) is then unfavoured, due to the instability of $\mathrm{Zn}(\mathrm{OH})_{2}$ at certain $\mathrm{pH}$ levels. Another suggestion is that the role of HMT may be one which specifically promotes the growth of nanowires [31] (rather than other shapes) and this will be discussed in section 2.2.3. 


\subsubsection{Crystal Nucleation}

An advantage of hydrothermal growth is the ability to control the location at which the final $\mathrm{ZnO}$ product, given in equation 2.4, forms into a crystal. This is achieved by defining an area of $\mathrm{ZnO}$ seed layer at which the $\mathrm{ZnO}$ crystals preferably form. The control and reproducibility of crystal growth is particularly important for the fabrication of devices.

There are two regimes under which the nucleation of crystals in solution may occur; homogeneous and heterogeneous nucleation. Homogeneous nucleation is the formation of crystallites within the growth solution, while heterogeneous nucleation occurs when crystallites form on some solid surface within the solution. The $\mathrm{ZnO}$ crystals formed through homogeneous nucleation precipitate as a white powder within the growth solution and are considered waste in regards to device manufacture. The crystals formed through heterogenous nucleation will grow on the predefined $\mathrm{ZnO}$ seed layer on the device surface and may be made use of.

The driving force behind the formation of crystals from solution is the minimisation of the Gibbs free energy [48]. In general the free energy of atoms in a bulk crystal is lower than that when dissolved in solution [49]. However, this is not necessarily the case for atoms near the surface of the crystal. The key difference that drives any preference between homogeneous and heterogeneous nucleation is the effective surface area of the crystal. In the case of homogeneous nucleation, the entire surface of the crystal is in contact with solution, while with heterogeneous nucleation only part of the crystallite's surface is exposed (the rest is in contact with a surface). The energetic effect of the area in contact with the solid surface will depend on the structural and chemical interaction of the nucleus and the surface. If the surface shares similar crystal properties (crystal structure, lattice parameter, etc), the overall effect will be a reduction in the free energy.

The primary result of heterogeneous nucleation is that in the presence of a seed crystal of $\mathrm{ZnO}$, crystallites will preferentially form on the seed. In this thesis sputtered layers of $\mathrm{ZnO}$ will be used as a seed layer. The $\mathrm{ZnO}$ nanowires will grow from this seed layer and hence their location can be controlled. This will be critical to optimising the device structure in chapter 5.

\subsubsection{Crystal Shape and Polyethyleneimine}

The hexagonal $\mathrm{ZnO}$ crystal has three primary growth directions [50]; $\langle 2 \overline{1} \overline{1} 0\rangle,\langle 0 \overline{1} 10\rangle$ and [0001]. These can be grouped to define either growth from the lateral faces of the hexagonal crystal $(\langle 2 \overline{1} \overline{1} 0\rangle$ and $\langle 0 \overline{1} 10\rangle)$ or longitudinal growth from the top face ([0001]) (see figure 2.2). It has been observed that growth in the longitudinal direction is favoured and has a faster growth rate than any of the other crystal directions [51]. This inevitably leads to the growth of long, thin crystals. 
The growth behaviour and shape of the $\mathrm{ZnO}$ crystals, however, is not fixed and can be manipulated to form a very large variety of nano structures. Structures reported in literature include belts, cages, combs, helices, propellers, rings, springs and wires [11]. These structures are produced by manipulating the growth rates in different crystal axes through the use of various chemical and environmental factors.

The hexagonal $\mathrm{ZnO}$ crystal consists of alternating layers of $\mathrm{Zn}^{2+}$ and $\mathrm{O}^{2-}$ ions (see section 2.1). The overall result is that the lateral faces will be effectively neutral, while the top/bottom faces will be slightly polar. The different faces will therefore interact with different chemical species. Non-polar molecules may adhere to the lateral faces, while polar ones may adhere to the top/bottom faces. The adhered molecules physically block incoming ions and forces growth to only be possible in certain crystal axes. The type and concentration of the additional compound allows a large variety of crystals with reproducible aspect ratios to be grown [52].

As mentioned in section 2.2.1, the exact role of HMT is still not well established. One proposal [31] is that HMT molecules may preferentially bind to the non polar lateral faces of the $\mathrm{ZnO}$ crystal. HMT therefore, does not necessarily act only as a chemical reactant, but may block lateral crystal growth. The adhesion of HMT leaves the top crystal face open to further incoming ions resulting in long, thin crystals or nanowires.

This thesis focusses on using $\mathrm{ZnO}$ for sensing applications. The sensing mechanism relies on surface interactions on the $\mathrm{ZnO}$ nanowires (The mechanism of sensing will be discussed in full in chapter 3). Therefore in order to produce a larger signal, a large surface to volume ratio is necessary. To achieve this, nanowires with large aspect ratios are required. To further increase the aspect ratio of the nanowires, a lateral growth blocking agent is used; polyethyleneimine (PEI) is a type of polymer made from repeating units of two aliphatic carbons and an amine group. PEI molecules can be either linear (repeating $\mathrm{CH}_{2} \mathrm{CH}_{2} \mathrm{NH}$ units ) or contain branches (which require $\mathrm{CH}_{2} \mathrm{CH}_{2} \mathrm{~N}$ units). Typical PEI polymers consist of tens of monomers and have a particularly high denticity. PEI provides a steric hindrance to lateral growth and is effective at promoting longitudinal growth. The addition of PEI has been shown to increase the aspect ratio of nanowires by a factor as large as four [26].

The addition of PEI in hydrothermal growth also has an impact on the chemical reactions that take place. This is most apparent in that in order to grow nanowires to a given length, a longer growth time is required when using PEI [53]. The exact chemical role of PEI has been closely examined by Liu et al. [47]. They have shown that PEI will chelate to $\mathrm{Zn}^{2+}$ ions in solution and then react with formaldehyde $\left(\mathrm{CH}_{2} \mathrm{O}\right)$, formed from the decomposition of HMT (equation 2.2), and release the $\mathrm{Zn}^{2+}$ ions back into solution [47]: 


$$
\begin{gathered}
\mathrm{Zn}^{2+}+\mathrm{PEI}-\mathrm{NH}_{2} \rightarrow \mathrm{PEI}-\mathrm{NH}_{2}-\mathrm{Zn}^{2+} \\
\mathrm{PEI}-\mathrm{NH}_{2}-\mathrm{Zn}^{2+}+\mathrm{CH}_{2} \mathrm{O} \rightarrow \text { PEI-NH}{ }_{2}=\mathrm{CH}_{2}+\mathrm{Zn}^{2+}
\end{gathered}
$$

These reactions complicate the chemistry of hydrothermal growth. The chelation of $\mathrm{Zn}^{2+}$ to PEI reduces the concentration of free $\mathrm{Zn}^{2+}$ ions for $\mathrm{ZnO}$ formation. The chelated $\mathrm{Zn}^{2+}$ will be gradually released into solution, meaning that it will take longer to complete the reaction. This in turn means that the crystal growth rate is slower and will likely have an effect on the crystal shape.

In summary, PEI can be used to promote growth along the $\mathrm{c}$ axis of $\mathrm{ZnO}$, producing longer, thinner nanowires. The mechanism by which PEI achieves this is complicated and may be a combination of steric hindrance of the lateral $\mathrm{ZnO}$ faces, as well as acting as a mediator of ions in the growth solution. The careful use of PEI will be used in this thesis to modify the structure of $\mathrm{ZnO}$ nanowires.

\subsection{Summary}

In this thesis, hydrothermal growth will be used to grow $\mathrm{ZnO}$ nanowires on substrates patterned with $\mathrm{ZnO}$ seed layers. The growth solution consists of zinc nitrate and hexamethylenetetramine in DI water. Polyethyleneimine will also be added to improve the aspect ratio of the nanowires. 


\section{Chapter 3}

\section{ZnO Nanowire Devices and Sensors}

This chapter covers the background information relevant to understanding the operation and capabilities of $\mathrm{ZnO}$ nanowire devices, with a focus on devices used for sensing. Many $\mathrm{ZnO}$ nanowire devices take the form of a field effect transistor (FET). Background theory relevant to the operation of FETs is covered in section 3.1. Section 3.2 covers the current capability of $\mathrm{ZnO}$ nanowire FETs and how their environmental interactions can be used to make sensors. Finally section 3.3 covers $\mathrm{ZnO}$ nanowires which are chemically functionalised to interact with biomolecules.

\subsection{Field Effect transistors}

A field effect transistor (FET) is a three terminal semiconductor device where the current flow between two terminals is controlled by the third. FETs effectively act as a switch with a conductive and an insulating regime. A full description of FETs can be found in [12].

An FET is constructed from a piece of semiconductor contacted to two terminals called the "drain" and the "source". In devices that do not exhibit any electrical polarity, the source is usually defined as the common terminal. A third terminal called the "gate" is separated from the semiconductor by a layer of insulating dielectric. The insulating layer stops the flow of current from the gate, however a voltage applied to the gate can induce band bending in the semiconductor [12]. A simple schematic of an FET is show in figure 3.1.

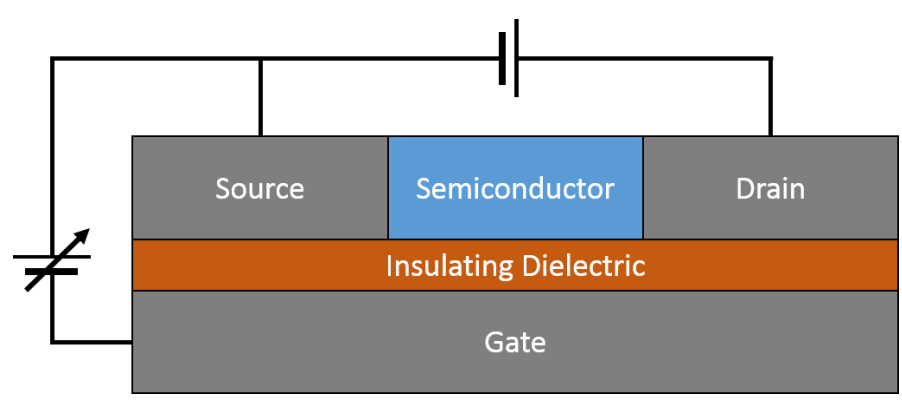

Figure 3.1: A simple schematic of an FET. Current passes between the drain and source electrodes via a piece of semiconductor. The semiconductor is separated from the third contact, the gate, by a layer of insulting dielectric. 


\subsubsection{Band Bending}

A idealised band diagram of an n-type FET is given in figure 3.2. At equilibrium the fermi energy in the semiconductor lies between its valence and conduction bands. Only thermally excited electrons are present in the conduction band, leading to a small (but non zero) conductivity [43].

When a positive voltage is applied to the metal (gate) the energy bands close to the insulator-semiconductor surface are bent down to lower energies [12]. The conduction band then passes below the fermi level and electrons fill these states. These electrons may then conduct along the semiconductor surface which increases its conductivity. This is called the accumulation case.

Alternatively, the depletion case is where a negative voltage is applied to the metal. This naturally has the opposite effect and bends the bands upwards. This reduces the concentration of any thermally activated electrons that may be present in the conduction band at the semiconductor surface, therefore, decreasing the conductivity of the semiconductor.

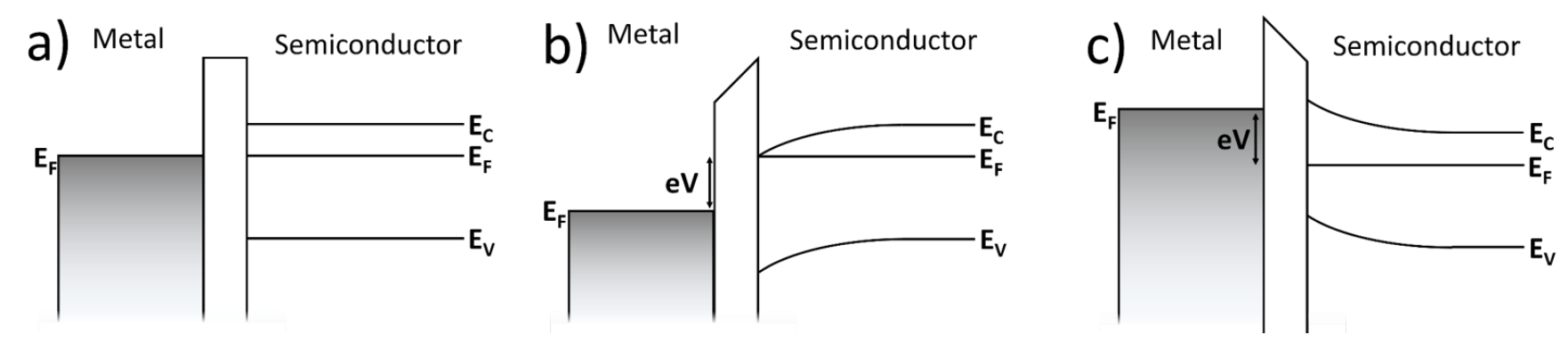

Figure 3.2: The idealised band diagram of an FET with the metal gate on the left and the (n type) semiconductor on the right, separated by a layer of insulator. a) equilibrium, b) accumulation case, c) depletion case.

\subsubsection{Liquid Gating}

An alternative to that described in section 3.1.1 is to use a liquid as the gate dielectric. This is required for many biosensing applications (i.e. for sensing in biological fluids) and therefore is pertinent to this thesis. In a liquid gated setup the liquid forms an electric double layer near the surface of the semiconductor [54-57]. This acts as the dielectric layer and screens the build up of charge on the semiconductor, due to the potential difference between the gate and the semiconductor. The electric double layer effectively behaves like a capacitor, allowing the accumulation of charge carriers as illustrated in figure 3.3.

The thickness of the electric double layer can be described using the Debye Length, $\lambda_{D}[55,56]$ : 


$$
\lambda_{D}=\frac{1}{\sqrt{4 \pi l_{B} \sum \rho_{i} z_{i}^{2}}}
$$

where $\rho_{i}$ and $z_{i}$ are the density and valency of the $\mathrm{i}^{\mathrm{th}}$ ion species respectively and $l_{B}$ is the Bjerrum length: the distance between two charges where their coloumb and thermal energies are equal. In water at $25^{\circ} \mathrm{C}$, the Bjerrum length is $0.72 \mathrm{~nm}$ [56] and so the Debye length can be approximated as [58]:

$$
\lambda_{D} \approx \frac{3.2 \times 10^{-10}}{\sqrt{I}}
$$

where I is the ionic strength. For ionic liquids the Debye length, and hence thickness of the electric double layer, is generally on the order of a few nanometres (eg. $1 \mathrm{mM}$ tris buffer has a Debye length of $9.6 \mathrm{~nm}$ [59]). The gate voltage drops almost entirely across the electric double layer and can achieve very high capacitances (several $\mu \mathrm{Fcm}^{-2}$ ) $[57,60]$. In less ionic solutions the Debye length is larger, such as in deionised water where the debye length is about $1 \mu \mathrm{m}$ [18] (note that even pure water has a hydronium/hydroxide ion concentration of $10^{-7} \mathrm{M}$ [61]). Even though the Debye length of DI water is relatively large compared to the typical thicknesses of solid dielectric layers (e.g. in this thesis $100 \mathrm{~nm}$ of $\mathrm{SiO}_{2}$ is used), the electric double layer in water can still achieve higher capacitances due to its larger dielectric constant ( 80 for water vs 4 for $\mathrm{SiO}_{2}$ [62]). In summary, the use of a liquid dielectric can often screen more charge build up in the semiconductor and induce a stronger field dependence.

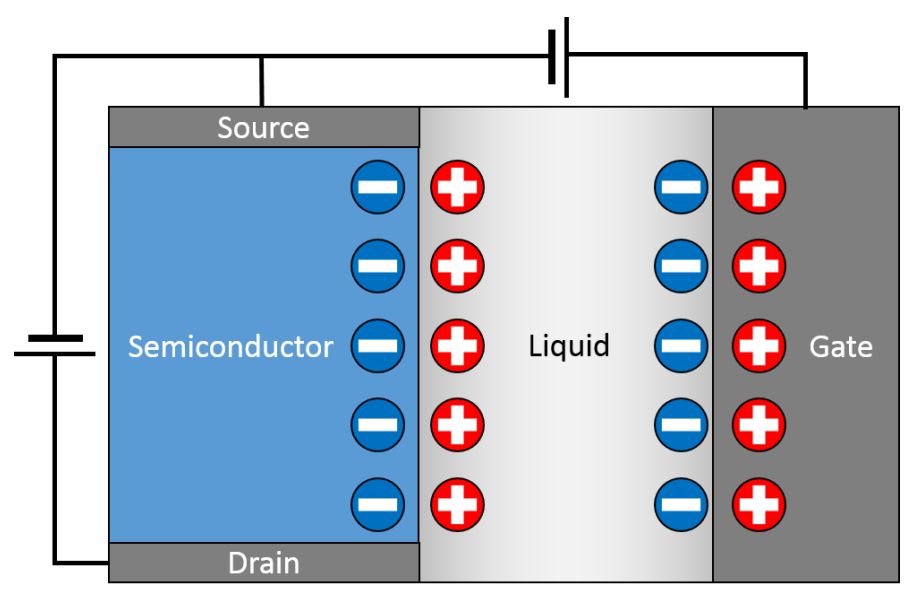

Figure 3.3: A simple schematic of the electric double layer that forms during liquid gating of an FET. Ions (+ and -) in the liquid are able to screen the build up of charges in the gate and in the semiconductor. 


\subsubsection{FET Measurements and Parameters}

The main electrical measurement used in this thesis is the transfer sweep. A number of parameters that describe the performance of an FET can be calculated from these sweeps and are described below.

\section{Transfer Sweep}

Transfer sweeps are often used to measure FETs. They involve setting a defined drain-source voltage, $\mathrm{V}_{D S}$, and then sweeping the gate voltage, $\mathrm{V}_{G}$, across a range of values. The drain-source current, $\mathrm{I}_{D S}$, and gate (or leakage) current, $\mathrm{I}_{G}$, can also be measured.

\section{On/Off Ratio}

An FET can be said to have an "off" state where the device is insulating and has a very low conductance. Likewise there is an "on" state where the device is conductive. Generally the on/off ratio is simply defined as the ratio between the maximum and minimum drain-source currents. This represents the conductivity ratio between the highly conductive and insulating regimes.

\section{Threshold Voltage}

The threshold voltage is loosely defined as the gate voltage at which the device starts to conduct. As some current will always flow in the devices, a further definition is necessary. There are many methods used to extract a threshold voltage, but a common one, and that which will be used in this thesis, is the linear extraction method $[63,64]$.

This method involves taking the transfer curve of a device, in linear scale, and curve fitting a straight line to the steepest part of the data. The line is then extrapolated back and the intercept with the $\mathrm{x}\left(\mathrm{V}_{G}\right)$ axis gives the threshold voltage (see figure $3.4 a$ ).

\section{Subthreshold Swing}

The subthreshold swing is a measure of switching "speed". It is a measure of the gate voltage required to change the drain-source current by one order of magnitude [64]. It is calculated by plotting the logarithm of the drain source against the gate voltage and fitting with a linear trend line (see figure 3.4b). The inverse of the gradient of this line is the subthreshold swing, usually expressed in $\mathrm{mV} /$ decade. 


\section{Transconductance}

The transconductance, $\mathrm{g}_{m}$, is another measure of switching speed. It is defined as the derivative of the transfer curve [64]:

$$
g_{m}=\frac{d I_{D S}}{d V_{G}}
$$

Normally the maximum transconductance is given, in units of siemens (S).
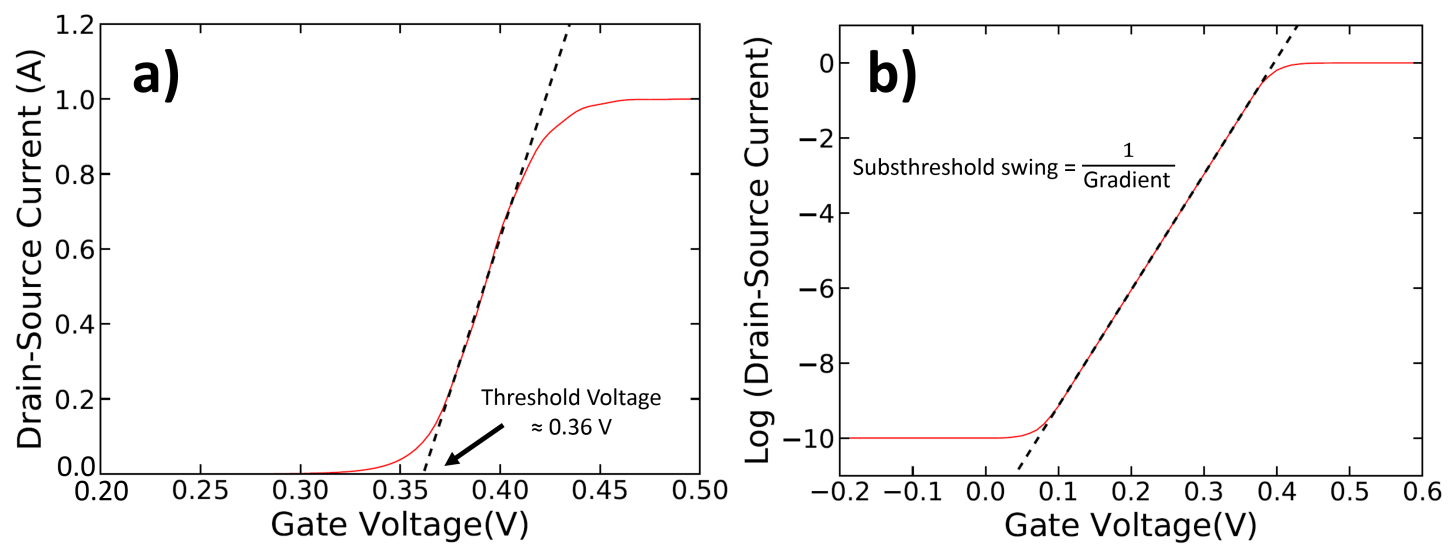

Figure 3.4: a) The linear fit of the transfer curve in linear scale is used to extrapolate the threshold voltage. b) The linear fit of the log of the drainsource current is used to calculate the subthreshold swing. This data also has an on/off ratio of $10^{10}$.

\subsection{ZnO Nanowire Devices}

$\mathrm{ZnO}$ nanowires have been used in many types of devices. This section covers a select few device types which are relevant to sensing applications. This should give the reader an understanding of the current capabilities of $\mathrm{ZnO}$ nanowire devices and the underlying mechanisms of sensing with $\mathrm{ZnO}$.

\subsubsection{Field Effect Transistors}

$\mathrm{ZnO}$ nanowires have been employed in FET devices using a large variety of synthesis techniques (as described in section 2.2) and nanowire geometries (e.g. single wires [65-70], multi wires [71-73], wire networks [74-79] and wire stacks [80]). There are a number of trends that can be identified across all of these devices. The 
$\mathrm{ZnO}$ nanowires show the expected $\mathrm{n}$ type behaviour, with the depletion mode occurring at more negative voltages. Furthermore, most devices exhibit the behaviour of a depletion-mode transistor [66-69,72-80]; that is, at gate voltages of $0 \mathrm{~V}$ the devices are in the "on" state, with threshold voltages ranging from -0.5 $\mathrm{V}$ [77] to $-26 \mathrm{~V}[68,69]$. On/off ratios have also been reported, with some reaching as large as $10^{7}[78,79]$. An example transfer sweep of a hydrothermally grown single $\mathrm{ZnO}$ nanowire FET is shown in figure 3.5a [68]. The device in figure 3.5a has an on/off ratio of over $10^{6}$ and is clearly a depletion-mode transistor, with a gate voltage of about $-40 \mathrm{~V}_{G}$ necessary to turn the device "off".

Most of the aforementioned FET devices are gated through a solid such as the back of the substrate or through a layer on top of the nanowires. An alternative, however, is to use a liquid as the gate dielectric, placed on top of the device. Less work has been done on this setup, but gating has been trialled through both deionised water [81] and ionic liquids [77]. These devices exhibit similar properties to the back gated devices. The devices still behave as depletion mode transistors, and exhibit on/off ratios up to $10^{5}[77,81]$. Switching of the FET from on to off, however, can be achieved over a much narrower gate voltage range (about \pm 1 or $2 \mathrm{~V}$ ) in comparison to dry ZnO nanowire FETS. Switching occurring over smaller gate voltages when wet is also seen in carbon nanotube FETs and is attributed to the strong capacitance of the electric double layer [57,82] (see section 3.1.2). An example of an FET that makes use of a network of $\mathrm{ZnO}$ nanowires gated in DI water is shown in figure 3.5b [81]. The FET switches from off to on in less than $2 \mathrm{~V}_{G}$ and with an on/off ratio of $10^{5}$.
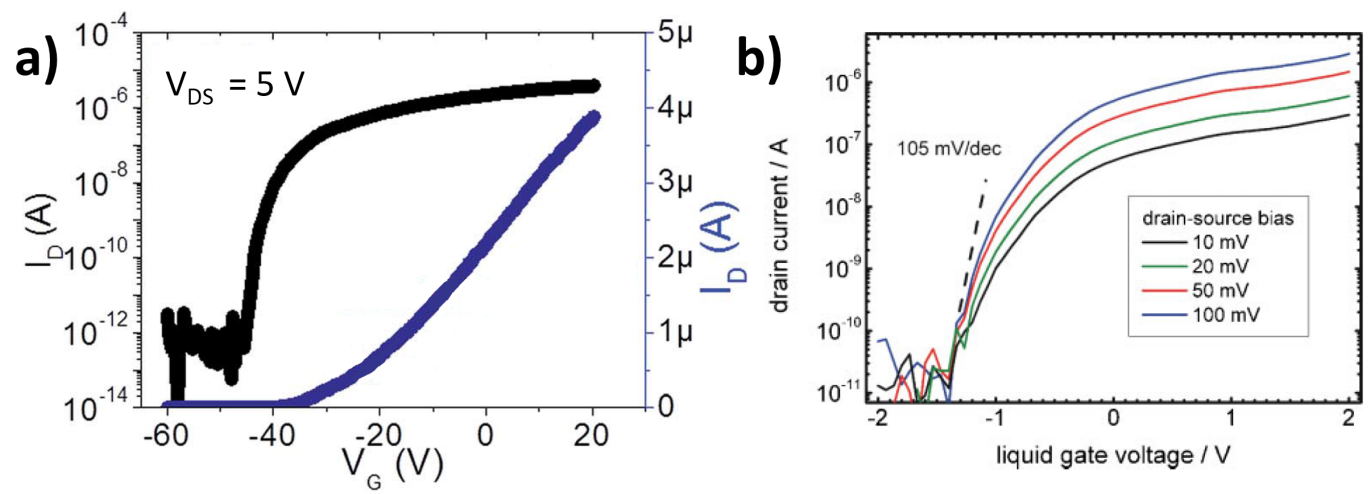

Figure 3.5: Example transfer sweeps of $\mathrm{ZnO}$ nanowire FETs. a) The transfer sweep of a dry single $\mathrm{ZnO}$ nanowire FET. The drain-source current is displayed in both log (black) and linear (purple) scale (reproduced from [68]). b) A ZnO nanowire FET gated in DI water. Switching occurs over a much narrower gate voltage (reproduced from [81]). 


\subsubsection{Gas Sensors}

The electronic behaviour of $\mathrm{ZnO}$ nanowires is heavily dependant on surface effects and the interaction with surface molecules. This makes $\mathrm{ZnO}$ a useful tool for sensing changes in the local environment as a chemiresistor, a device that changes it's resistance due to chemcial interactions with the environment. $\mathrm{ZnO}$ nanowire gas sensors have been utilised in the sensing of gases such as $\mathrm{O}_{2}[66,74,83]$, water vapour (humidity) [84-88], ethanol [89-97], $\mathrm{CO}[89,90,98], \mathrm{NO}_{2}$ [91,98], $\mathrm{NH}_{3}[89,90,98], \mathrm{H}_{2}[96], \mathrm{H}_{2} \mathrm{~S}[89,90]$, LPG $[89,90]$, gasoline $[89,90]$ and formaldehyde $[89,90]$.

It is known that oxygen molecules can be adsorbed on the surface of $\mathrm{ZnO}$ and accept electrons from $\mathrm{ZnO}[83,89,91,93]$. Various species of oxygen ions $\left(\mathrm{O}_{2}^{-}, \mathrm{O}^{-}\right.$ and $\mathrm{O}^{2-}$ ) are then formed on the surface of $\mathrm{ZnO}$, which reduces the charge carrier concentration. This results in a decrease in the conductivity of the nanowire and hence a smaller drain source current. The adsorption of $\mathrm{O}_{2}$ is a reversible process (due to diffusion) and therefore the conductivity of $\mathrm{ZnO}$ can be related to the $\mathrm{O}_{2}$ content of the environment. This behaviour has been shown to have a considerable effect on $\mathrm{ZnO}$ nanowires. The partial pressure of oxygen changes the overall conductivity of the device $[66,74]$. Higher oxygen partial pressures leads to smaller drain-source currents, by as much as an order of magnitude [66], and also results in more negative threshold voltages in FETs (by up to $4.5 \mathrm{~V}$ ) [74].

Other chemicals can react with or be adsorbed by $\mathrm{ZnO}$. The effect each gas has on the $\mathrm{ZnO}$ depends on whether the gas is oxidative or reductive. Oxidative gases such as $\mathrm{NO}_{2}$ and $\mathrm{NH}_{3}$ [98], can adsorb to the $\mathrm{ZnO}$ surface and withdraw electrons from the $\mathrm{ZnO}$ bulk, reducing the conductivity. Alternatively reductive gases, such as ethanol $[91,93,94]$ or $\mathrm{CO}[98]$, can react with surface oxygen ions and release electrons back into the $\mathrm{ZnO}$, increasing the conductivity. An example of oxidative $\mathrm{NO}_{2}$ and reductive $\mathrm{CO}$ sensing with a single $\mathrm{ZnO}$ nanowire, fabricated using a chemical vapour deposition technique, is given in figure 3.6 [98].

The sensitivity of these chemical sensors can be defined by the ratio of the conductivity of the device when exposed to chemical vapour divided by the conductivity when the device is exposed to a reference atmosphere (usually just ambient atmosphere). Humidity sensors have been particularly successful with sensitivities reported as large as $10^{5}$ [86] (over $7 \%$ to $95 \%$ relative humidity). In general, however, $\mathrm{ZnO}$ nanowire sensors of other chemicals do not achieve as high sensitivities. Ethanol sensors based on $\mathrm{ZnO}$ nanowires have achieved sensitivities as large as 900 [96] (for 1000 ppm of ethanol). Unlike humidity sensors though, ethanol sensors, and most $\mathrm{ZnO}$ nanowire gas sensors, must be heated to high temperatures (typically $200^{\circ} \mathrm{C}-300^{\circ} \mathrm{C}[83,89,91-97]$ ) in order to produce a measurable signal. The sensitivity of $\mathrm{ZnO}$ nanowire FETs is also strongly dependant on the nanowire radius, with wires of smaller radii being more sensitive (larger change in conductivity) $[74,98]$. The dependence on radius is a clear indication that the interaction with gases is a surface effect. 

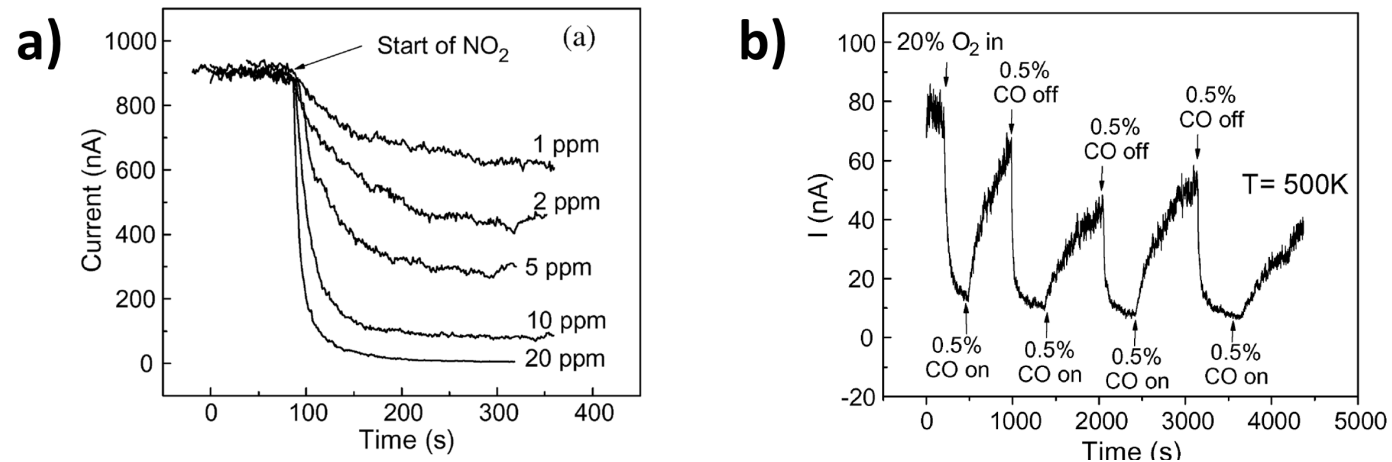

Figure 3.6: Example data of gas sensing using a $\mathrm{ZnO}$ single nanowire FET. a) $\mathrm{NO}_{2}$ is oxidative and reduces the conductivity of, and hence current through, the nanowire. b) $\mathrm{CO}$ is reductive and has the opposite effect. Note that in the $\mathrm{CO}$ measurement the device has been heated to $500 \mathrm{~K}$. Figures reproduced from [98].

\subsubsection{Photo Sensors}

The desorption of oxygen ions can also be controlled using UV radiation. Incident UV radiation can generate electron hole pairs within $\mathrm{ZnO}$ [99]. A hole can then recombine with an oxygen anion and consequently an oxygen molecule is adsorbed. UV induced desorption has been observed in ZnO nanowire FETs [44,71] with drain-source currents increasing by up to a factor of $10^{5}$ while under UV illumination (see figure 3.7) [100]. The intensity of UV can then be measured as a function of drain-source current. The desorption of oxygen due to UV irradiation has also been shown to be repeatable and fast, with photoresponse times (the time it takes currents to stabilise when illumination is turned on/off) of about $2 \mathrm{~s}$ [71].

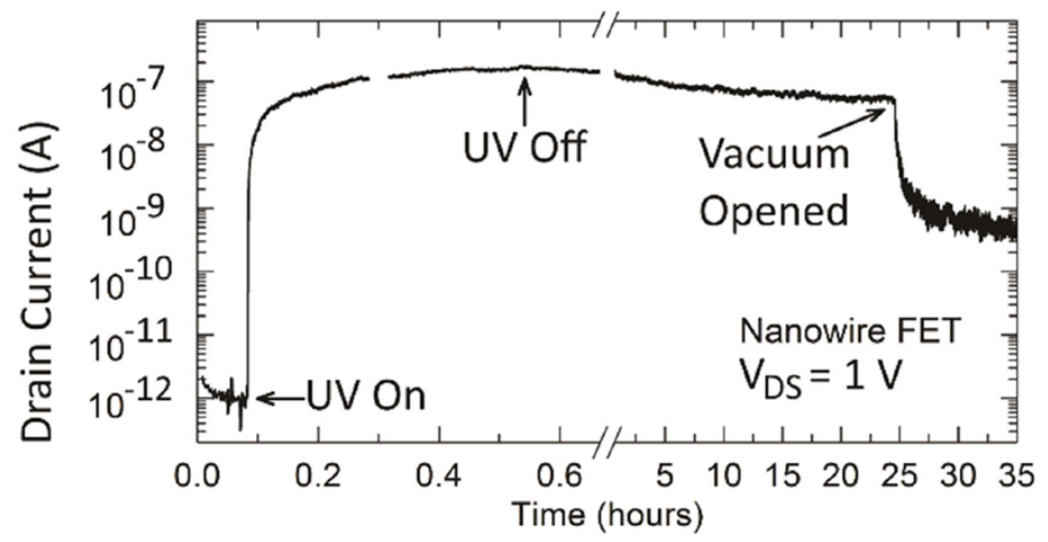

Figure 3.7: Response of a $\mathrm{ZnO}$ nanowire FET to UV illumination while under vacuum. UV irradiation forces the ejection of oxygen molecules from the surface of $\mathrm{ZnO}$. This releases free electrons into the $\mathrm{ZnO}$ and the conductivity increases. Once the vacuum is opened the $\mathrm{ZnO}$ comes in contact with oxygen molecules which readsorb to the surface and reduce the conductivity. Figure reproduced from [100]. 


\subsubsection{Hysteresis in ZnO Nanowire FETs}

$\mathrm{ZnO}$ devices often show hysteresis [66,101-104], that is the electrical properties of the device depend on the history of the device (such as how it was measured previously). Goldberger et al. [66] showed that the presence of adsorbed charge trapping species (in particular water molecules) on the surface of $\mathrm{ZnO}$ single nanowire FETs, had a major effect on their hysteretic behaviour. Hysteresis caused by adsorbed water molecules has also been observed in FETs constructed from nanowires of Germanium [105], InAs [106, 107] and $\mathrm{In}_{2} \mathrm{O}_{3}: \mathrm{Zn}$ [108], $\mathrm{MoS}_{2}$ thin films [109] and carbon nanotubes [110,111]. The water molecules can be directly bound to the surface of the semiconductor or on the surface of the $\mathrm{SiO}_{2}$ layer of the substrate $[105,108,110]$. The applied gate voltage polarises the adsorbed water molecules, which have a large dipole moment, and can screen the build up of charge on the semiconductor $[106,108,111]$. The lifetime of the dipole is on the order of a minute $[106,108,111]$ and so can persist beyond that of the applied gate voltage. The persistent dipoles of the water molecules result in a time dependant effective gating of $\mathrm{ZnO}$ nanowires. This leads to differences in the behaviour of the forward (negative gate voltage to positive voltage) and backward (positive gate voltage to negative voltage) sweeps of a transfer curve. The adsorbed water molecules on $\mathrm{ZnO}$ nanowires can be removed with vacuum or a $\mathrm{N}_{2}$ atmosphere which reduces or removes any hysteresis. However the water molecules can readsorb once the device is exposed to air $[66,104]$.

\subsection{Biofuncionalsiation}

The biofunctionalisation of sensors sees the addition of receptor molecules to the surface of the active component of a device. This allows the system to then interact with biomolecules and hence is vital for sensing [13-17]. Biosensors often make use of oligonucleotide aptamers (short single strands of DNA or RNA) to capture target molecules [18]. Aptamers bind to target molecules (analytes) with a very high level of specificity, allowing for accurate detection of analytes, without interaction with other species present in the environment [112-114]. Furthermore a large variety of aptamers may be synthesised [112], each with their own target molecule.

\subsubsection{Aptamers}

RNA sequences were first artificially synthesised using in-vitro selection and amplification in 1990 and were shown to specifically bind to target molecules $[112,113]$. These short strands were termed aptamers and later work was done to fabricate DNA aptamers in a similar manner [114]. The structure of DNA was established in the 1950s [115] and consists of repeating units of four different bases; 
Adenine (A), thymine (T), cytosine $(\mathrm{C})$ and guanine $(\mathrm{G})$ (RNA is similar, but replaces thymine with uracil (U)). The bases are then held together by a backbone of alternating phosphate and sugar units, as depicted in figure 3.8, with the ends referred to as $3^{\prime}$ and 5'. Two strands of DNA or RNA may form a double helix through hydrogen bonding between bases. The binding between bases is highly selective and only occurs between specific pairs: Adenine and thymine (or uracil in RNA), and cytosine and gyanine. There are four main types of nucleic acids; single stranded RNA (ssRNA), single stranded DNA (ssDNA), double stranded RNA (dsRNA) and double stranded DNA (dsDNA) (further classifications exist, but are not relevant here). In this thesis single stranded DNA aptamers will be used.

The principle behind aptamer based sensing is that upon binding of the target molecule (analyte) to the aptamer, the aptamer reconfigures and induces a change in potential on the surface of the semiconductor it is linked to. When an aptamer is linked to a device, it typically takes the form of a loose random coil. If an analyte comes into contact with the aptamer, however, the aptamer will wrap around the analyte forming a much more ordered structure. Two common structures are the G-quadruplex structure (potassium aptamer) [116] and tertiary folding (as seen in the cocaine aptamer) [117]. These two structures are presented in figures 3.9a and $\mathrm{b}$. The aptamer backbone contains negatively charged phosphate groups (see figure 3.8) and when the aptamer reconfigures these groups move closer to the surface of the semiconductor (for example the free potassium aptamer may extend up to $8.7 \mathrm{~nm}$ from the semiconductor surface, but once bound to a $\mathrm{K}^{+}$ion it reconfigures and is held within $1.5 \mathrm{~nm}$ of the surface [118]). The proximity of these charges to the semiconductor results in a change in the electrostatic environment and effectively acts like a gate voltage. The strength of this quasi-gating depends on the number of aptamers that are bound to analytes and is therefore related to the analyte concentration.

FET biosensors infer the concentration of an analyte from the drain source current. The current depends on the field effect induced by the reconfiguration of the surface bound aptamers as a result of them binding to analytes.

\subsubsection{ZnO Biosensors}

$\mathrm{ZnO}$ has been previously used in biosensing FETs, both in the form of thin films [120-123] and as nanowires [19,103,124-126]. The adhesion of aptamers to ZnO typically requires a linker molecule. This linker binds at one end to the $\mathrm{ZnO}$ surface (often via a silanisation reaction with surface hydroxyl groups), while the other end binds to the aptamer (often through an amine or thiol group at the 5' end of the aptamer). $\mathrm{ZnO}$ has been successfully functionalised using a number of linker molecules including (3-glycidoxypropyl)trimethoxysilane (GOPS) [124], (3-glycidoxypropyl)dimethylethoxysilane [121] and aminopropyltriethoxysilane (APTES) [123]. 


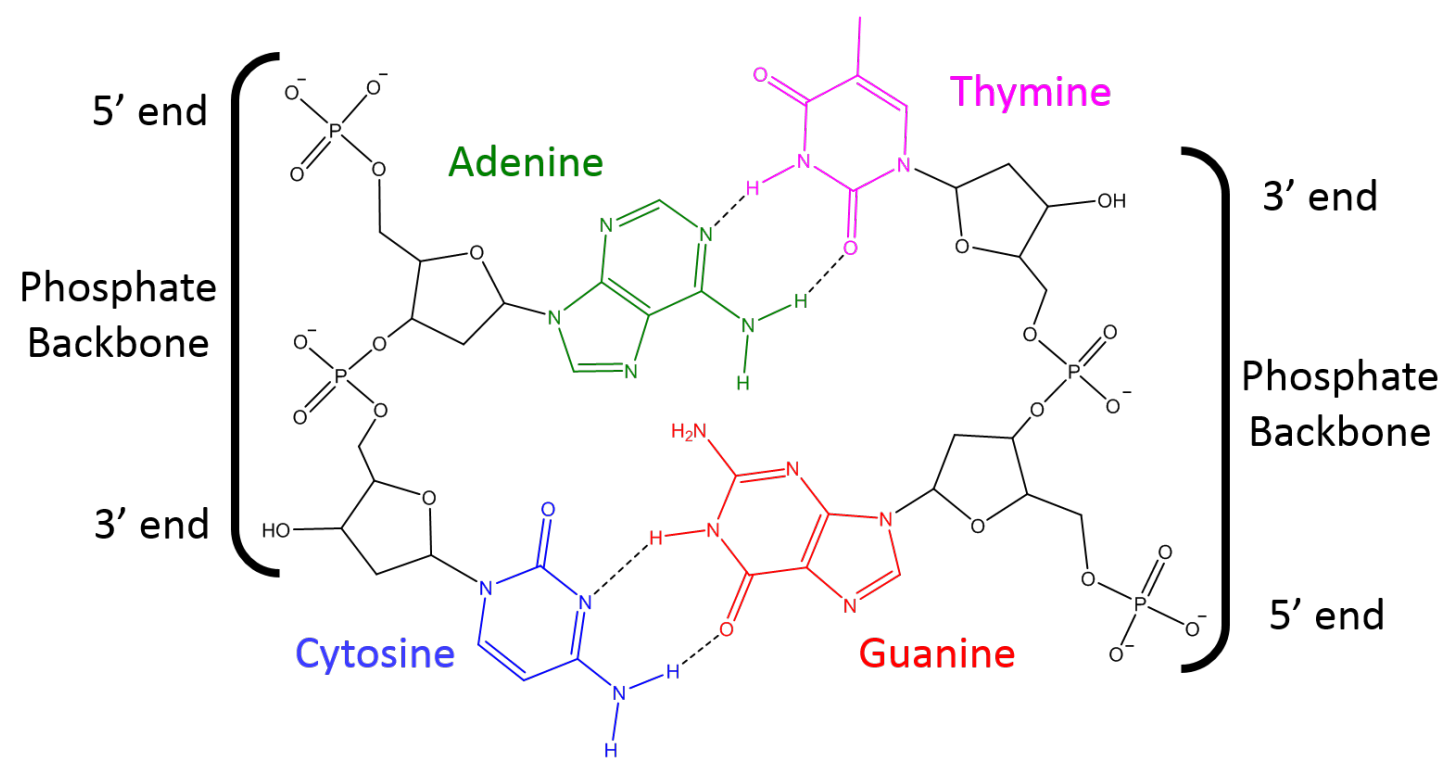

Figure 3.8: This figure shows a section of double stranded DNA, formed from two single DNA strands held together by hydrogen bonding (dashed lines). DNA contains four different bases; adenine(green), thymine (purple), cytosine (blue) and guanine (red), connected by a phosphate backbone. In the case of RNA, uracil replaces thymine. Uracil is identical to thymine, except for the removal of the methyl group on the heterocyclic ring.
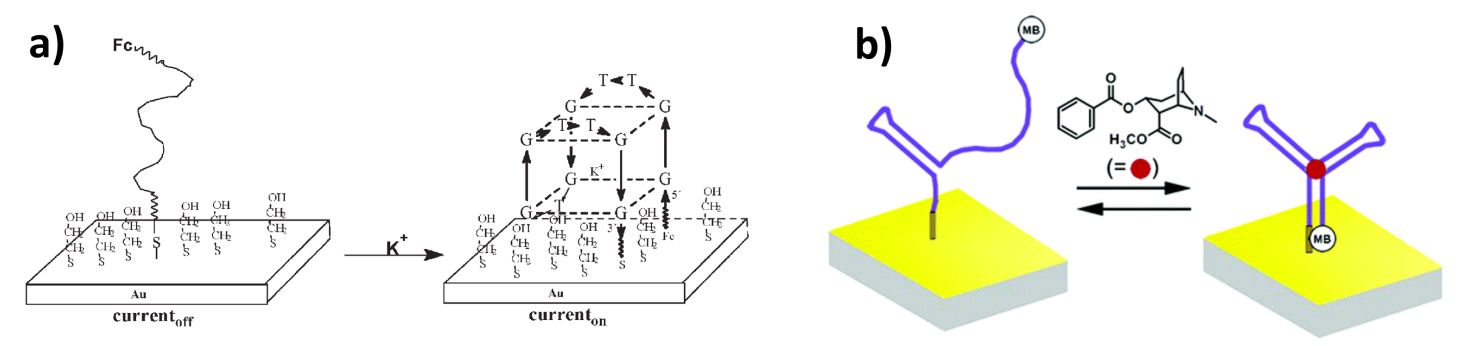

Figure 3.9: Diagrams of the reconfiguration of aptamers upon binding to analytes. a) The free potassium aptamer is relatively straight (left), but once bound to $\mathrm{K}^{+}$it forms the cube like G-quadruplex structure. Figure reproduced from [116]. b) The cocaine aptamer is partially folded when free, but once bound to a cocaine molecule it forms a three way junction structure. Figure reproduced from [119].

One molecule that has been successfully sensed using a $\mathrm{ZnO}$ thin film FET is riboflavin [120,121]. Hagen et al. functionalised the surface of the $\mathrm{ZnO}$ thin films with the corresponding riboflavin aptamer. The detection of riboflavin was demonstrated for concentrations between $1 \mathrm{pM}$ and $10 \mu \mathrm{M}$ of riboflavin in ultra pure water. In addition, these devices were tested for the sensing of 2-quinoxaline carboxylic acid [120], a molecule very similar in structure to riboflavin. No response was measured and is a clear measure of the specificity of the riboflavin aptamers. 
The specificity of aptamers to complimentary strands of DNA has also been demonstrated on $\mathrm{ZnO}$ nanowires by Leiterer et al. [19]. These $\mathrm{ZnO}$ nanowires were fabricated using the vapour-liquid-solid method and functionalised with aptamers, using GOPS as the linker molecule. The nanowires were then exposed to either the complementary or non-complementary DNA strand in $5 \mathrm{x}$ phosphate-buffered saline. Only the complimentary DNA strands bound to the aptamers functionalised on the $\mathrm{ZnO}$ nanowire surface (detected using the fluorescence of a group attached to the aptamers). The functionalised $\mathrm{ZnO}$ nanowires exposed to non-complimentary DNA show no measurable difference to unexposed control samples, implying a $100 \%$ rejection of the non-complimentary DNA.

As described in section 3.2.2, $\mathrm{ZnO}$ will readily interact with many different chemical gases. The lack of selectivity means that gas sensing in an uncontrolled environment is challenging, as the signal induced in the $\mathrm{ZnO}$ nanowires will be a function of all gases present, not just the intended target chemical. Biofunctionalisation, however, can partially overcome this challenge by increasing the specificity for certain molecules. ZnO thin film FETs functionalised with the riboflavin aptamer have been used in the sensing of gaseous riboflavin by Hagen et al. [122]. The measurements were carried out under a controlled nitrogen atmosphere with a small concentration of riboflavin vapour. Detection of riboflavin was achieved at concentrations of $309 \mathrm{nM}$ (or $0.116 \mathrm{ppm}$ ). It is yet to be demonstrated, however, if this detection signal is greater than that caused by variations in the ambient atmosphere and whether it is viable in an uncontrolled atmosphere.

Functionalisation has also been successful with other organic molecules, such as biotin, in place of aptamers. Choi et al. [125] fabricated single ZnO nanowire FETs using the vapour-liquid-solid method and functionalised the surface of the $\mathrm{ZnO}$ nanowires with biotin. Biotin has a high affinity for streptavidin and the sensing of strepavidin in phosphate buffer was demonstrated. These nanowire devices were demonstrated to become saturated (i.e. upper limit for sensing) at $250 \mathrm{nM}$ and could detect as low as $2.5 \mathrm{nM}$ (although this was identified as not necessarily the lower limit). Kim et al. [103] also demonstrated a similar result with single $\mathrm{ZnO}$ nanorod FETs fabricated using metalorganic vapour phase epitaxy. These devices were also functionalised with biotin and the sensing of streptavin, in phosphate buffer, between concentrations of $25 \mathrm{nM}$ and $25 \mu \mathrm{M}$ was demonstrated.

\subsection{Summary}

FETs operate by inducing band bending in a semiconductor and causing the depletion or accumulation of charge carriers in the conduction band. $\mathrm{ZnO}$ thin films and nanostructures can be used in the fabrication of FETs and there are numerous examples of their application as sensors. ZnO Biosensors have been fabricated by functionalising the surface of $\mathrm{ZnO}$ with aptamers, which allow for highly selective analyte detection. The reactions between $\mathrm{ZnO}$ and chemical vapours has also been used to make $\mathrm{ZnO}$ chemiresistors operate as gas sensors. 


\section{Chapter 4}

\section{ZnO Nanowire Device Fabrication and Char- acterisation}

This chapter describes in detail the experimental methods, processes and equipment used in the work presented in this thesis. All experiments were conducted in the cleanroom fabrication facility at the School of Chemical and Physical Sciences at Victoria University of Wellington.

\subsection{ZnO Nanowire Device Fabrication}

\subsubsection{Substrate Preparation}

All devices referred to in this thesis were fabricated using used $365 \mu \mathrm{m}$ thick $\mathrm{p}$ type silicon (i.e. doped with Boron) as the substrate. The silicon is supplied as three inch silicon wafers with a $100 \mathrm{~nm}$ thick native oxide layer (Silicon Quest International). The oxide acts as an insulating layer between the conductive silicon and the device that will be produced on top. There should, therefore, be no electrical connection between the $\mathrm{ZnO}$ device and the Si. Regardless, however, $\mathrm{p}$ type silicon is specifically chosen so that it contrasts with $\mathrm{ZnO}$ (which is expected to be $\mathrm{n}$ type [6]). This means any measurement of any semiconducting behaviour due to the Si can then be quickly identified. Furthermore, the oxide layer also acts as the dielectric layer when gating through the back of the Si chip (see section 3.1).

Before cleaving the wafers, the $\mathrm{SiO}_{2}$ layer is protected with a physical barrier of photoresist and polydimethylsiloxane (PDMS). The layer of photoresist is sacrificial and can be easily removed with acetone. The PDMS layer provides a thick, spongy layer which protects the $\mathrm{SiO}_{2}$ from any scratching. First AZ1518 photoresist (Microchem) is spun on to the wafer at $900 \mathrm{rpm}$ for 40 seconds. This is then baked on a hotplate for five minutes at $95^{\circ} \mathrm{C}$. The PDMS is prepared by mixing a 1:10 ratio of elastomer curing agent and silicone base (Sylgard 184 Incor). Mixing introduces a number of air bubbles into the PDMS, which are subsequently removed by desiccation for 30 minutes. The PDMS is then spun onto the wafer (again at $900 \mathrm{RPM}$ for 40 seconds) and baked at $90^{\circ} \mathrm{C}$ for one hour.

Once the protective layers are dry, the back face of the Si is scored into one centimetre squares with a diamond tipped pen. The Si can then be accurately cleaved along these scores, the PDMS layer is simply peeled off and the photoresist removed with acetone. 


\subsubsection{Photolithography}

The purpose of photolithography is to selectively and accurately cover a substrate with a predetermined pattern of a material (or materials). This process is used to make the patterned $\mathrm{ZnO}$ seed layer and electrodes on the $\mathrm{Si} / \mathrm{SiO}_{2}$ substrates.

Photolithography makes use of chemicals known as photoresists. A photoresist is a photosensitive resin which changes its chemical behaviour upon UV exposure. By using a mask parts of the photoresist can be expose to UV and then a developer solution can remove either the exposed or unexposed photoresist (depending on the photoresist's properties). This can be done with a resolution on the scale of microns.

AZ1518 is spin coated onto the $\mathrm{Si} / \mathrm{SiO}_{2}$ substrates for one minute at $4000 \mathrm{RPM}$ and baked on a hot plate at $95^{\circ} \mathrm{C}$ for two minutes. The substrates are then aligned under a mask and exposed to $25 \mathrm{mWcm}^{-2}$ (at a wavelength of $365 \mathrm{~nm}$ ) of UV radiation from a mercury lamp for 7.5 seconds. The UV changes the chemical nature of the exposed photoresist which can then be removed by developing in AZ326 developer (Microchem, diluted at a ratio of 1:3 AZ326:DI water) for 20 seconds. Areas of photoresist that were exposed to UV are removed and a corresponding pattern of the mask is left behind in the form of solid photoresist. This process is presented in figures $4.1 \mathrm{a}$ ) - d).

All of the photolithography was completed using a Karl Suss MJB3 mask aligner, fitted with a USHIO SHP 350W lamp and a Laurell Model WS-400 BZ-6NPP/Lite spincoater. The masks were designed using LayoutEditor and fabricated at Callaghan Innovation.

\subsubsection{Sputtering}

The substrates patterned with photoresist are first deposited with a layer of $\mathrm{ZnO}$ and then a layer of $\mathrm{Ti}$. The $\mathrm{ZnO}$ provides a seed layer for $\mathrm{ZnO}$ nanorods/ wires to nucleate on (see section 2.2.2). The top Ti layer has two functions. First it acts as a conductive surface that can be used as an electrode to which a good electrical contact can be made. Second, the Ti layer obscures the top face of the $\mathrm{ZnO}$ layer and means that only the side profile of $\mathrm{ZnO}$ is exposed. During hydrothermal growth, this restricts nanorod/nanowire growth to the sides of the $\mathrm{ZnO} / \mathrm{Ti}$ stack.

Radio-frequency (RF) sputtering was used to deposit the $\mathrm{ZnO}$ and Ti layers. Sputtering is a physical vapour deposition process. It involves taking a target of the material that is to be deposited and bombarding it with a plasma of gas ions. The plasma is formed by applying a high voltage between the target and a cathode. A magnetron is then used to localise the plasma and direct it onto the target. 
a)

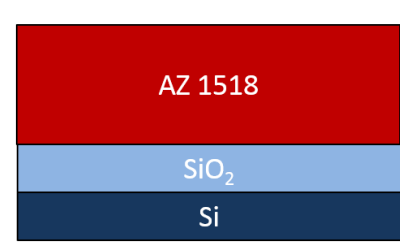

d)

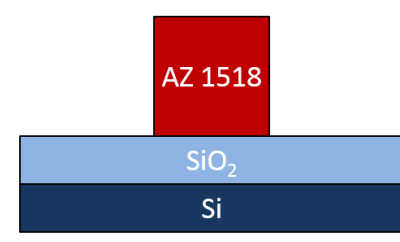

b)

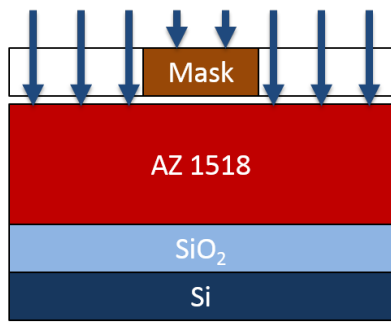

e)

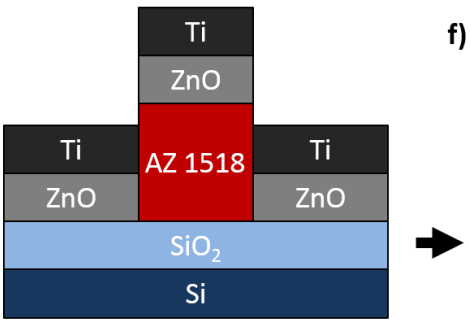

c)
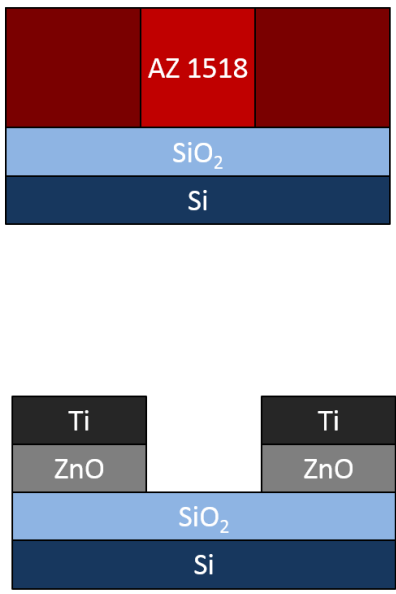

Figure 4.1: The photolithography process for AZ 1518: a) AZ1518 photoresist is spincoated onto the $\mathrm{Si} / \mathrm{SiO}_{2}$ substrate. b) The sample is placed under a mask and exposed to UV radiation. c) The UV radiation induces a chemical change in the exposed photoresist. d) AZ326 developer is used to removed the exposed photoresist. e) $\mathrm{ZnO}$ and $\mathrm{Ti}$ are sputtered onto the patterned substrates. f) The remaining AZ 1518 is removed with acetone and the $\mathrm{ZnO} / \mathrm{Ti}$ layers on top are lifted off. Not to scale.

By directing the plasma onto the target, the ions collide with the target at very high energies. The collisions cause atoms to be ejected from the surface of the target. These atoms then travel from the target and land on the interior of the sputter coater chamber. Substrates placed within the chamber will therefore have a layer of the target material deposited on them. For a more in depth explanation of sputtering theory, refer to [127].

To deposit the $\mathrm{ZnO}$ and Ti layers, an HHV Auto500 sputter coater, powered by an Advanced Energy Cesar 136 600W RF generator was used. This system allows up to three different targets to be loaded at any one time and each can be sputtered independently. This means that the Ti layer can be deposited immediately after the $\mathrm{ZnO}$ and without having to vent the chamber.

The chamber is initially evacuated to a pressure of less than $5 \times 10^{-9}$ Bar. It is then back-filled with instrument grade argon gas (BOC, $\mathrm{Ar}>99.99 \%$ ) to a pressure of $1 \times 10^{-5}$ Bar. A $200 \mathrm{~nm}$ thick layer of $\mathrm{ZnO}$ (target: $99.99 \% \mathrm{ZnO}$, Plasmaterials) is first deposited at a $\mathrm{V}_{\text {bias }}$ of $250 \mathrm{~W}$, followed by $100 \mathrm{~nm}$ of Ti (target: $99.99 \% \mathrm{Ti}$, Plasmaterials) at a $\mathrm{V}_{\text {bias }}$ of $200 \mathrm{~W}$.

After the sputter deposition the substrates are bathed in acetone. The remaining photoresist is dissolved and the $\mathrm{ZnO}$ and Ti layers above are lifted off. The sputtering and liftoff processes are shown in figures $4.1 \mathrm{e}$ ) and f). 


\subsubsection{Acid Etch}

Before hydrothermal growth the substrates were etched with acid. The acid etches the $\mathrm{ZnO}$ layer and leaves an overhang of the Ti layer. The overhang acts as a physical barrier to vertical nanowire growth and promotes lateral growth. This is discussed in full in chapter 5. $1 \mathrm{mM}$ phosphoric acid $\left(\mathrm{H}_{3} \mathrm{PO}_{4}\right)$ and 0.89 hydrochloric acid were prepared in DI water. Both acids have the same $\mathrm{pH}$ of 3.05 [62]. A substrate is dipped into one of the acids for a specified time (a few seconds) and then immediately rinsed in DI water and blown dry with $\mathrm{N}_{2}$ gas.

\subsubsection{Hydrothermal Growth}

The $\mathrm{ZnO}$ nanowires are grown using hydrothermal growth as described in section 2.2.

A $25 \mathrm{mM}$ growth solution is prepared by combining $1.859 \mathrm{~g}$ of $\mathrm{Zn}\left(\mathrm{NO}_{3}\right)_{2} \cdot \mathrm{H}_{2} \mathrm{O}(98$ $\%$ purity, Sigma Aldrich) and 0.876 g of hexamethylenetetramine (HMT) (99\% purity, Sigma Aldrich) in $250 \mathrm{ml}$ of DI water (resistivity $\geq 18.2 \mathrm{M} \Omega \mathrm{cm}$ ). The solution is shaken in a borosilicate glass bottle until all the solids are dissolved and the solution is clear. $1.6 \mathrm{~g}$ of polyethylenimine (PEI, $800 \mathrm{gmol}^{-1}$, Sigma Aldrich) is then added to provide a PEI concentration of $8 \mathrm{mM}$. The bottle is placed into a water bath held at $95^{\circ} \mathrm{C}$ so that the growth solution may preheat for 1 hour. After the growth solution is preheated, the substrates are added and left for 19 hours.

During hydrothermal growth there is a large amount of homogeneous nucleation, resulting in a white precipitate (i.e. $\mathrm{ZnO}$ ) forming throughout the growth solution. The $\mathrm{ZnO}$ precipitate sinks and it is important that the sample does not become covered in precipitate. To avoid this, the substrates are mounted on a glass slide with Kapton tape. When the substrates are added, the glass slide is dropped into the bottle such that it rests at an angle, with the substrates facing down (see figure 4.2). This avoids any precipitate settling on the substrate.

\subsubsection{Scanning Electron Microscope}

High magnification imaging was necessary for measurement and calibration of the nanowires and sputtered $\mathrm{ZnO} / \mathrm{Ti}$ layers. A Jeol 6500F scanning electron microscope (SEM) was used for all micrographs presented in this thesis. 


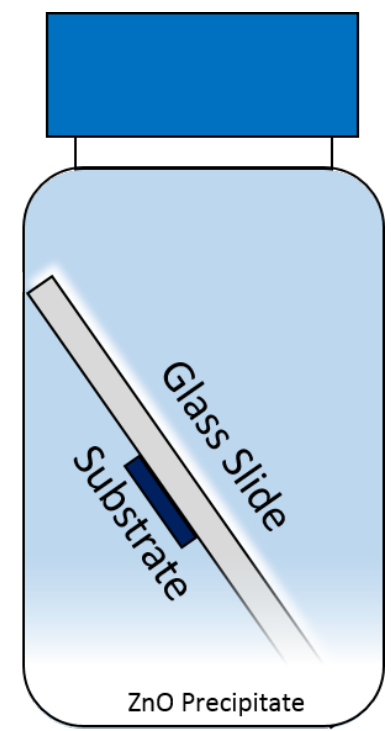

Figure 4.2: the substrates are attached to a glass slide and dropped into the solution such that they face down. The white $\mathrm{ZnO}$ precipitate settles on any of the up facing surfaces

\subsubsection{Encapsulation}

Ideally the leakage current of an FET should be zero, but in reality this is not always easy to achieve. To minimise leakage currents the samples are coated in an insulating layer of photoresist. By using photolithograhpy (see section 4.1.2) the photoresist layer may be applied such that the active area of nanorods / nanowires are still exposed. This ensures that only the key, active area of the device is exposed to liquid and reduces the number of possible current paths. This is particularly important for liquid gated setups, due to the possibility of conduction between the electrodes and the liquid (see section 4.3.2).

Samples are encapsulated using SU-8 photoresist (Microchem). SU-8 is used as it is hardier and more chemically resistant than AZ 1518. Furthermore, SU-8 layers can be fabricated with thicknesses on the order of one to hundreds of microns, while still producing edge features with high aspect ratios [128].

This procedure is very similar to that for AZ 1518 (see section 4.1.2). The SU-8 is first diluted in cyclopetanone at a ratio of 2:1. The diluted SU-8 is spincoated onto the sample for 10 seconds at 500 RPM, followed by 40 seconds at 4000 RPM. The sample is prebaked for a series of sequential temperatures; $55^{\circ} \mathrm{C}, 85^{\circ} \mathrm{C}, 55^{\circ} \mathrm{C}$, off, each lasting three minutes. A mask is used to obscure the electrode contacts and channels of the samples. SU-8 is a negative resist and so the areas of photoresist that are not exposed to UV will be removed during development (this is the opposite behaviour to AZ 1518). The sample is aligned under the mask and exposed to UV $\left(25 \mathrm{mWcm}^{-2}\right.$ at $\left.365 \mathrm{~nm}\right)$ for 7.5 seconds. This is followed by a post bake, identical to the prebake, except with temperatures $5^{\circ} \mathrm{C}$ lower. The sample is developed in propylene glycol mono methyl ether acetate (PGMEA, Microchem) 
for 40 seconds and rinsed in isopropyl alcohol (IPA) for 10 seconds. Finally a hard bake is used to ensure the stability of the SU-8. The sample is baked on a hot plate at $200^{\circ} \mathrm{C}$ for 15 minutes and then allowed to cool for 30 minutes.

The final SU-8 layer is $6.5 \pm 0.2 \mu \mathrm{m}$ thick and a schematic of a fully fabricated sample is presented in figure 4.3 .

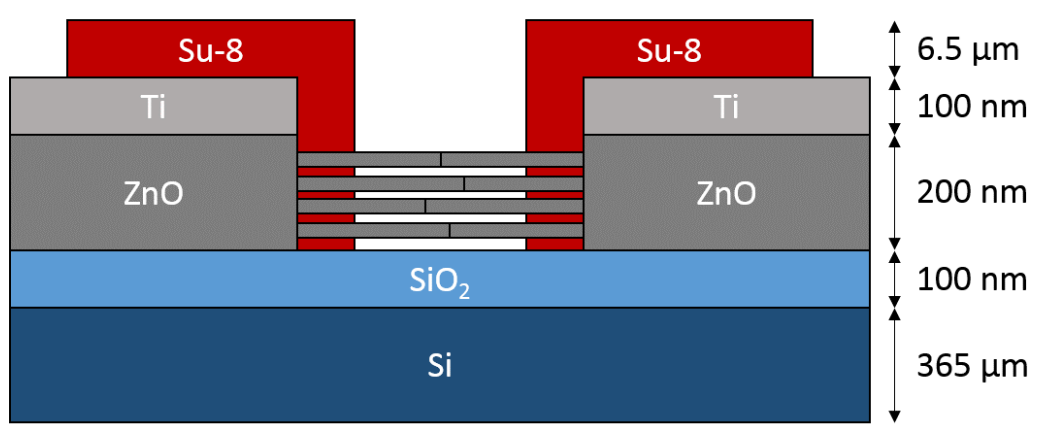

Figure 4.3: A schematic of the cross section of a fully fabricated device. The $\mathrm{ZnO}$ layer acts as the seed layer for the $\mathrm{ZnO}$ nanorods/nanowires. The Ti layer inhibits nucleation on the top face of the $\mathrm{ZnO}$ layer and also acts as an electrode. The SU-8 layer covers the whole sample except for a small gap over the channel and at the electrodes. Not to scale.

\subsection{Aptamer Functionalisation}

The method used in this thesis to achieve selectivity in biosensing is functionalisation with aptamers (see section 3.3). The surface of $\mathrm{ZnO}$ cannot be directly linked to commonly available aptamers and requires a linker molecule. The silane (3glycidyloxypropyl) trimethoxysilane (GOPS) is used as the linker. Organo silanes react with metal oxides and can form covalent bonds to the surface of $\mathrm{ZnO}$. GOPS also includes a chain terminating in an epoxy group. Epxoy groups readily react with amines and so an amine terminated aptamer can be bound to the GOPS modified $\mathrm{ZnO}$ surface. The chemical procedure is described in detail below and is adapted from $[19,124]$. A schematic of the reaction is given in figure 4.4 .

In order to ensure that GOPS reacts fully with the $\mathrm{ZnO}$, the $\mathrm{ZnO}$ surface needs to be fully oxygenated with reactive oxygen groups $\left(\mathrm{O}^{2-}, \mathrm{O}^{-}\right.$and $\mathrm{OH}^{-}$ $[124,129,130])$. This is achieved with a Plasma Etch PE-50 Compact Benchtop Plasma Cleaning System. The plasma cleaner is evacuated to below $200 \mathrm{mTorr}$ and then backfilled with $20 \mathrm{cc} /$ min of instrument grade oxygen gas $\left(\mathrm{BOC}, \mathrm{O}_{2}>\right.$ $99.7 \%$ ). The plasma cleaner is first run for one minute to ensure that the chamber is clean of any contaminants. Afterwards the chamber is vented, the samples are placed inside and the procedure repeated, except that plasma is run for 30 minutes. 
The next step is to react GOPS with the oxygenated $\mathrm{ZnO}$ surface. A $10 \mathrm{mM}$ solution of GOPS in anhydrous toluene is preprepared in a nitrogen filled glove box $\left(\mathrm{O}_{2}<5 \mathrm{ppm}, \mathrm{H}_{2} \mathrm{O}<0.5 \mathrm{ppm}\right)$. The sample is placed in the solution, with stirring, for at least six hours. The sample is rinsed in toluene and then hexane, before blowing dry with $\mathrm{N}_{2}$.

When not in use, amine terminated aptamers (AlphaDNA) are stored in a freezer at $-20^{\circ} \mathrm{C}$. The aptamers must be defrosted by diluting to a concentration of $1 \mathrm{mM}$ with $2 \mathrm{mM}$ tris (trisaminomethane) buffer ( $2 \mathrm{mM}$ tris buffer contains $0.04728 \mathrm{~g}$ trizma hydrochloride (Sigma Aldrich) in $200 \mathrm{ml}$ DI water) and then denatured by placing in an oven at $70^{\circ} \mathrm{C}$ for five minutes. The aptamer solution is pipetted onto the GOPS modified sample and left for two hours. Finally, the sample is rinsed in tris buffer, then DI water, and dried with $\mathrm{N}_{2}$ gas.

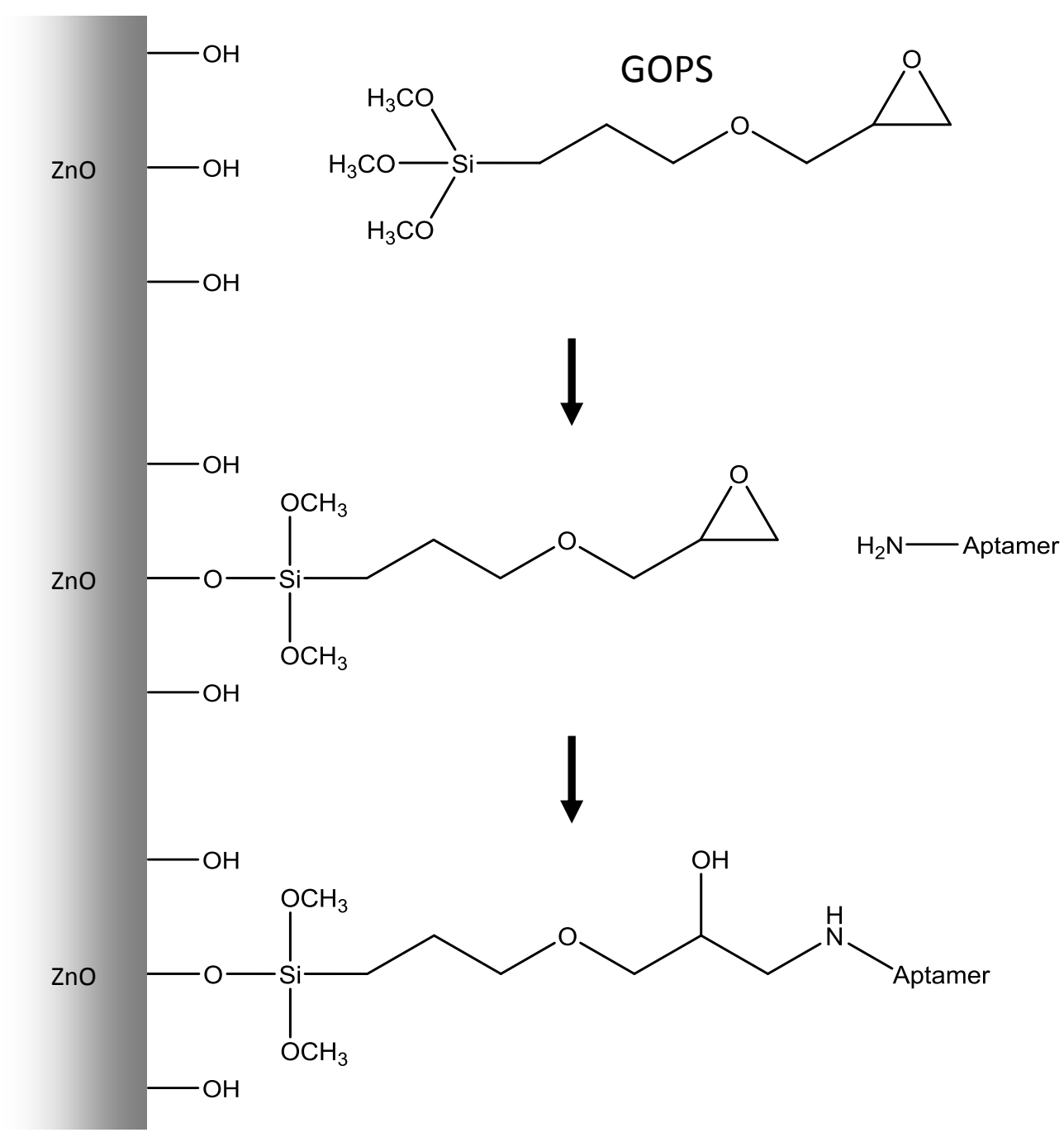

Figure 4.4: The chemical reactions involved in the aptamer functionalistaion of $\mathrm{ZnO}$. First GOPS binds to $\mathrm{ZnO}$ through the silane end. The exact chemical bonding between $\mathrm{ZnO}$ and the $\mathrm{Si}$ atom is debated and the silane may actual bind in a tridentate arrangement [131]. Secondly the amine terminated aptamer binds to the epoxy group of GOPS. 


\subsection{Electrical Measurements}

All electrical measurements were made using an Aglient 4156C Precision Semiconductor Parameter Analyzer. This allows for measurements at maximum resolutions of 1 femtoamp and 0.2 microvolts. Titanium probes are used to make a physical contact onto the surface of the Ti electrodes patterned on devices. Accurate contact is achieved using a Rucker and Kolls Probe Station and micromanipulators. The probes are linked to the parameter analyser by coaxial and triaxial cables to reduce electrical noise.

\subsubsection{Backgate Setup}

For the backgated setup the gate lead (from the parameter analyser) is soldered to a copper topped piece of fibreglass. This is held onto the probe station by a vacuum and samples are placed on top. The back of the Si substrate is in electrical contact with the copper top and hence the gate. A schematic of the set-up is shown in figure 4.5 .

\subsubsection{Liquid and Topgate Setup}

When measuring a sample in wet conditions the liquid must be contained so that it does not run off the sample or interfere with electrical measurements by coming into contact with the unencapsulated electrodes or probes. This is achieved with a polydimethylsiloxane (PDMS) well.

The PDMS is prepared by mixing a 1:10 ratio of elastomer curing agent and silicone base (Sylgard 184 Incor). The PDMS is poured into a clean Petri dish to a depth of $5-10 \mathrm{~mm}$, covered and left to set overnight. Once the PDMS is set it can be cut into rectangles with holes stamped through the middle. The PDMS wells can be placed on top of a sample and filled with liquid, while leaving the electrodes exposed at the sides.

The sample can be gated through the substrate (which is in contact with the copper pad) or alternatively a topgate setup can be achieved using a $\mathrm{Ag} / \mathrm{AgCl}$ electrode inserted into the liquid. The $\mathrm{Ag} / \mathrm{AgCl}$ electrode is positioned using a micromanipulator and inserted such that the entire surface of the electrode is immersed in liquid. An insulated wire runs from the top of the electrode and can be clipped onto the gate lead. Figure 4.6 shows a schematic of the liquid setup for both backgate and topgate configurations. 


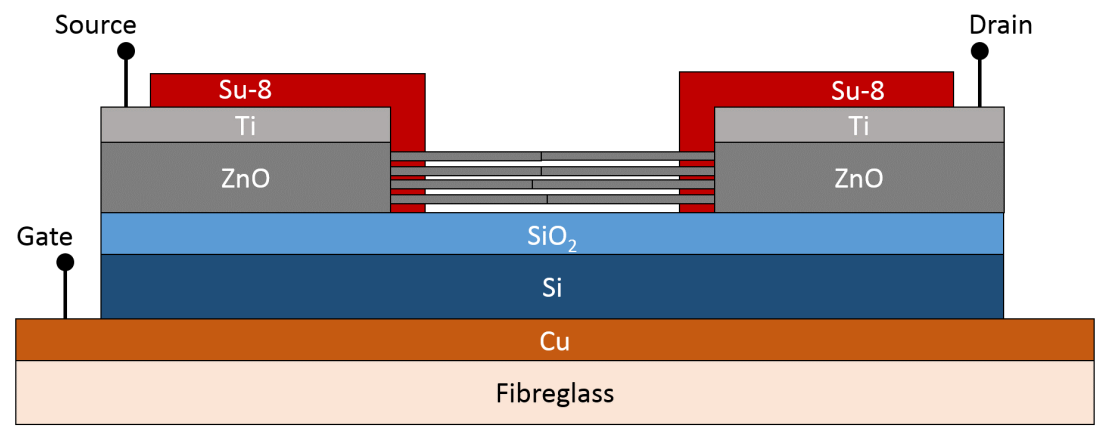

Figure 4.5: A schematic of a backgated sample. The sample is placed on top of a copper topped fibreglass pad connected to the gate lead of the parameter analyser. Not to Scale.

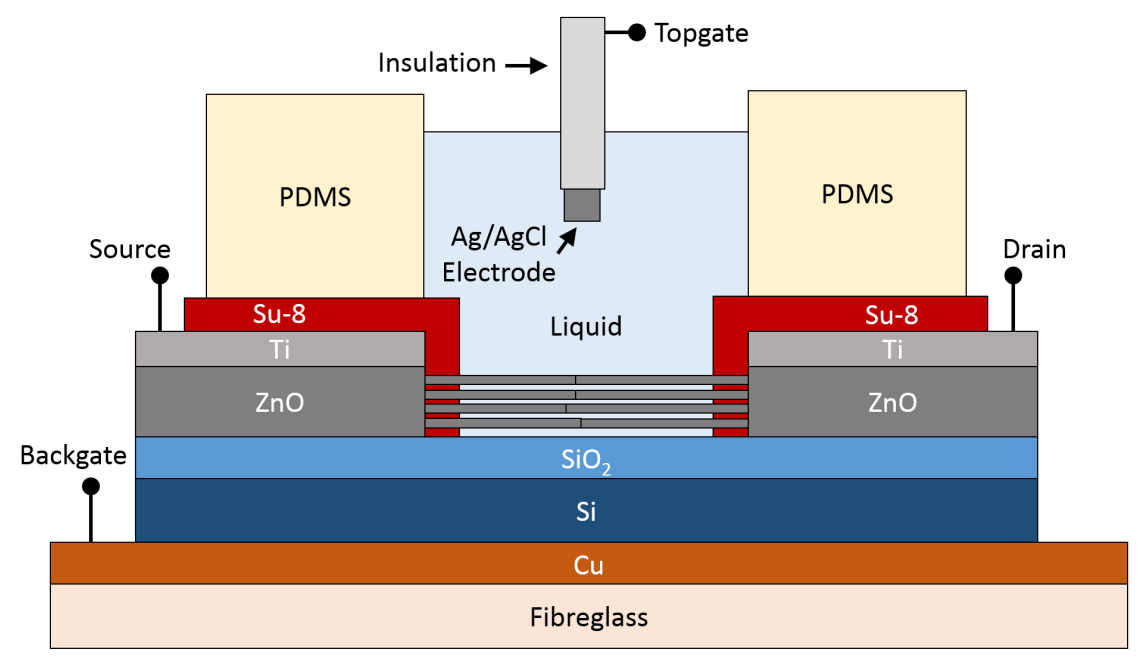

Figure 4.6: Schematic of a liquid gated sample. The liquid is contained in a PDMS well and the drain and source contacts can be made without contact with the liquid. The sample can be gated either through the copper pad underneath the sample or through the $\mathrm{Ag} / \mathrm{AgCl}$ electrode inserted into the liquid. Not to scale.

\subsection{Gas Rig}

Gas sensing measurements require the sample to be enclosed in a controlled atmospheric environment. To achieve this an in-house built gas sensing rig is used. A schematic of the gas rig is shown in figure 4.7. The sample is mounted onto a printed circuit board (PCB) using Kapton tape and connections made to individual devices with gold wire (diameter $=37 \mu \mathrm{m}$ ), adhered with conductive silver paint (RS Components). The PCB is mounted inside the gas sensing chamber and the headers (attached to the PCB) extend to the outside of the chamber. Connections are then made between the parameter analyser and the PCB headers using coaxial cables. 
The gas rig controls the flow of gas with mass flow controllers, operated via a custom LabView programme. Two gas lines (the vapour and dilution lines) are employed, each with a maximum flow of $100 \mathrm{sccm}$. Both lines are driven with oxygen free, industrial grade nitrogen (BOC, $\mathrm{N}_{2}>99.99 \%$ ). The vapour line, however, passes through a bubbler of the chemical to be detected. This introduces chemical vapour into the vapour line. The ratio of flow from each line can be modified to allow for different concentrations of chemical vapour.

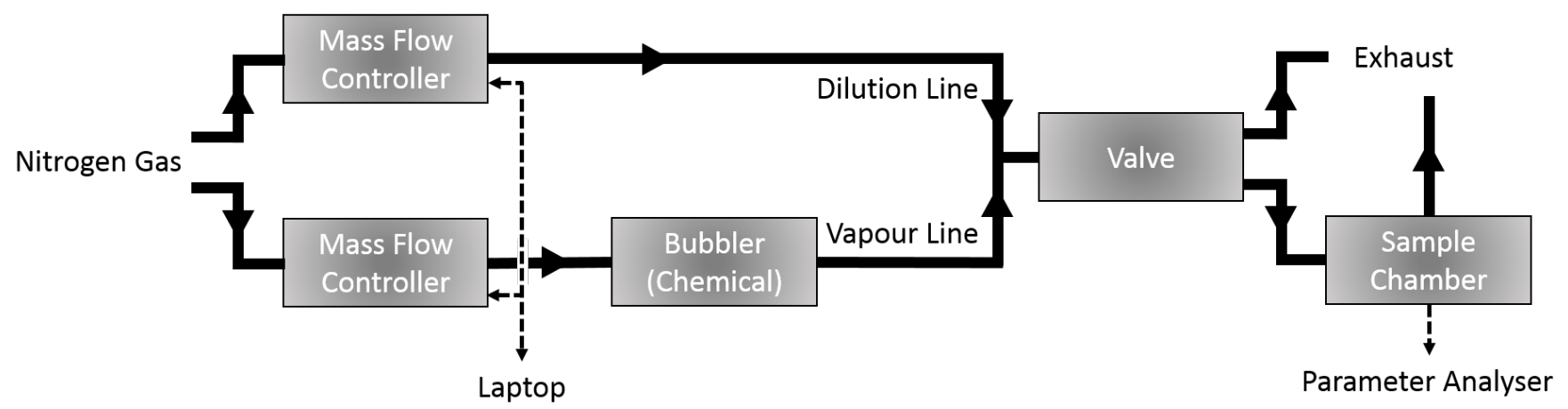

Figure 4.7: A schematic of the gas rig. The mass flow controllers are operated with the laptop and control the flow of the vapour and dilution lines. Gas can either be directed straight to exhaust or through the sample chamber. Electrical measurements are made insitu with the parameter analyser.

\subsection{Summary}

This chapter covers all the experimental techniques and measurement procedures used in this thesis. It can be used as a reference for the experiments that are used to produce the results presented in chapters $5,6,7$ and 8 . 


\section{Chapter 5}

\section{ZnO Nanowire Structure and Morphology}

This chapter deals with controlling the growth of $\mathrm{ZnO}$ nanowires and the optimisation of the design of devices. The effect of an acid etch on the $\mathrm{ZnO}$ seed layer and the resulting nanowire structure is investigated. Two types of devices, labelled as nanorods and nanowires, are fabricated and their structure described.

There does not seem to be any consensus in the literature as to the exact difference between a nanorod and a nanowire. In general nanorods are bigger than nanowires, but the two terms are used interchangeably [132]. In this thesis the term nanorods refers to the larger $\mathrm{ZnO}$ crystals with a diameter of $400 \pm 130 \mathrm{~nm}$, while nanowires refers to those that are $20 \pm 5 \mathrm{~nm}$ in diameter.

\subsection{Lateral Wire Growth}

To investigate the growth of $\mathrm{ZnO}$ nanowires from a $\mathrm{ZnO}$ seed layer, samples were prepared as described in section 4.1. Electrodes were patterned onto the $\mathrm{Si} / \mathrm{SiO}_{2}$ substrates using photolithography and sputtered with $200 \mathrm{~nm}$ of $\mathrm{ZnO}$ and 100 $\mathrm{nm}$ of Ti.

During hydrothermal growth, as described in section 2.2.2, the $\mathrm{ZnO}$ seed layer acts as a nucleation point for new $\mathrm{ZnO}$ crystals to form. Due to the Ti capping layer, only the side of the $\mathrm{ZnO}$ seed layer is exposed. Therefore, in the absence of any impurities or other nucleation sites, the wires grow exclusively from the edge of the $\mathrm{ZnO} / \mathrm{Ti}$ stack (see figure 5.2). This means that the wires can be grown in specific locations defined by the patterned $\mathrm{ZnO} / \mathrm{Ti}$ stacks. The use of a $\mathrm{Ti}$ capping layer has been successfully used in previous work at VUW $[44,100]$ and this method has also been used in literature with layers of $\mathrm{Ti} / \mathrm{Pt}[71], \mathrm{Cr}[133,134]$, Sn [134] or photoresist [72].

Figure 5.1 shows an SEM micrograph of the cross section of a sample grown using a 19 hour growth (with a 1 hour preheat) in a $25 \mathrm{mM}$ growth solution, with 8 $\mathrm{mM}$ PEI at $95^{\circ} \mathrm{C}$ (as described in section 4.1.5). Nanorods are clearly present and have grown exclusively from the exposed edge of the $\mathrm{ZnO}$ layer. The direction in which the wires grow, however, shows no directional growth preference. This results in a larger hemispherical distribution.

The hemispherical distribution is not optimal for the intended biosensing FET devices. The conduction path between the source and drain will be through the 
more lateral wires which form an electrical connection between them. The more vertical wires, which do not make connections, are therefore superfluous and do not provide a conduction path. Furthermore, they may also have the adverse effect of shielding the lateral wires from both the effects of gating and any chemical reactions during the functionalisation process. It is also intended that the devices be encapsulated with a photoresist layer, which reduces leakage currents in the device. The wires are longer $(20 \mu \mathrm{m})$ than the thickness of typical encapsulation layers (such as the $6.5 \mu \mathrm{m}$ SU-8 layer used in this thesis). The vertical wires may therefore pierce or damage the encapsulation layer, resulting in undesirable increases to the leakage current.

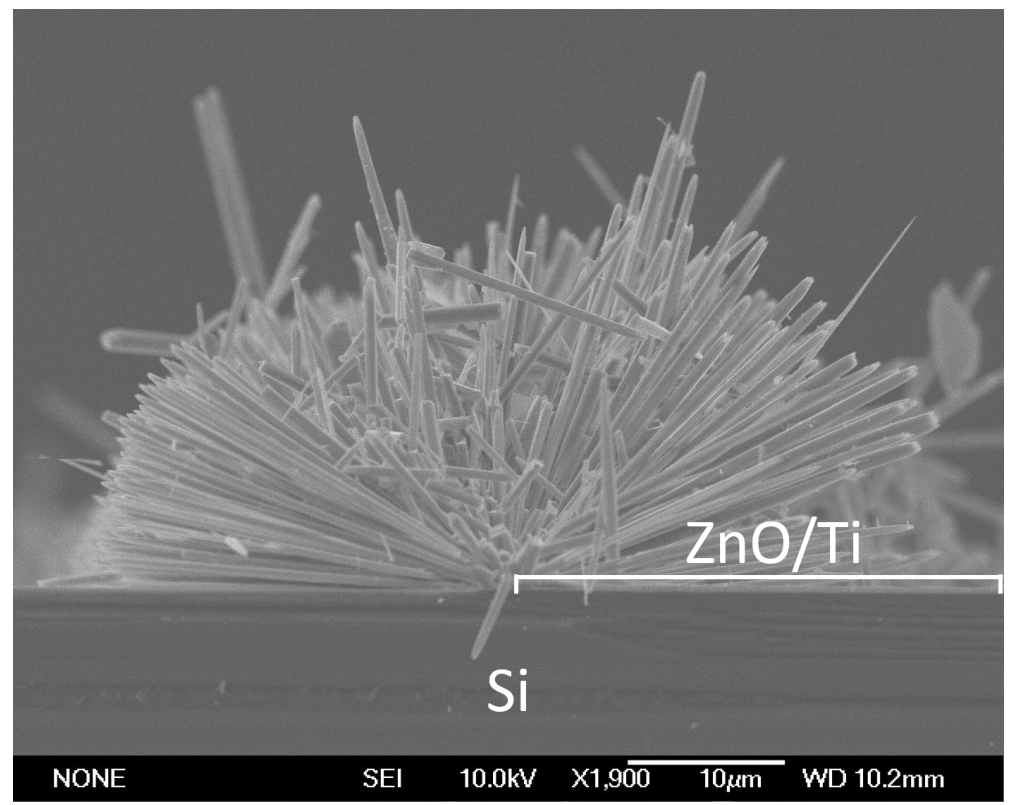

Figure 5.1: The standard wire growth produces a large hemispherical structure of nanorods. The nanorods all grow from the edge of the $\mathrm{ZnO} / \mathrm{Ti}$ stack (These layers are just visible as a faint grey line on the right hand side of the image). The $\mathrm{SiO}_{2}$ layer is not visible at this magnification.

a)

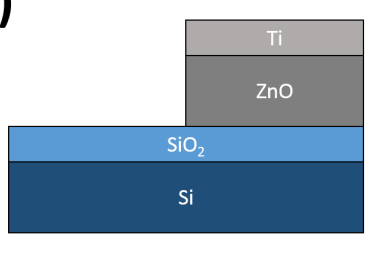

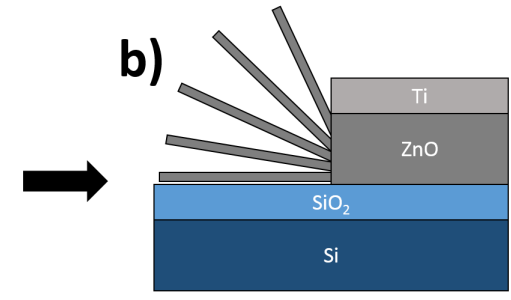

c)

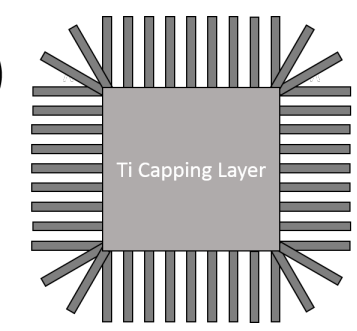

Figure 5.2: a) A schematic of the cross section of the sputtered layers. The $\mathrm{ZnO}$ layer is only exposed at the side of the $\mathrm{ZnO} /$ Ti stack. b) During hydrothermal growth, nanowires grow out from the exposed side of the $\mathrm{ZnO}$ layer. c) A schematic of the top down view of a $\mathrm{ZnO} / \mathrm{Ti}$ stack. Nanowires only grow out from the edge of the sputtered layers where the $\mathrm{ZnO}$ is exposed. Not to scale. 


\subsubsection{Acid Etch of ZnO Seed Layer}

A number of techniques exist to produce devices with exclusively lateral $\mathrm{ZnO}$ nanowires, but these typically involve depositing pregrown $\mathrm{ZnO}$ nanowires. Sun et al. [75] produced FETs with films of $\mathrm{ZnO}$ nanorods where the nanorods were pregrown in solution, using a hydrothermal growth method and then deposited onto devices using spin coating. Zhu et al. [135] grew arrays of vertical $\mathrm{ZnO}$ nanowires (using physical vapour deposition) onto a primary substrate and then wiped the nanorods onto a secondary substrate. The wiping breaks off the vertical wires and leaves them flat on the secondary substrate surface. These methods are not particularly suitable, however, as they do not allow accurate deposition and/or alignment of nanowires in predefined locations.

An alternate method is simply to etch the $\mathrm{ZnO}$ seed layer in acid before hydrothermal growth. $\mathrm{ZnO}$ is amphoteric [6], which means that it is highly soluble in acids and bases. Ti on the other hand is generally resistant to acids. Exposure to acid will etch the $\mathrm{ZnO}$ seed layer and leave an overhang of the Ti layer as depicted in figures $5.3 \mathrm{a}$ ) and $\mathrm{b}$ ). This overhang acts as a physical barrier that only allows for the growth of nanorods that are orientated parallel to the substrate/Ti layer (figure 5.3c). The hydrothermal growth of $\mathrm{ZnO}$ nanorods on etched $\mathrm{ZnO}$ seed layers has been used previously in the literature, but only when growing shorter nanorods of a length of 3 to $10 \mu \mathrm{m}[71,133]$. Whether this method is still viable for larger, longer nanorods is yet to be determined.

Two acids were trialled; $1 \mathrm{mM}$ phosphoric acid $\left(\mathrm{H}_{3} \mathrm{PO}_{4}\right)$ and $0.89 \mathrm{mM} \mathrm{HCl}$. $\mathrm{H}_{3} \mathrm{PO}_{4}$ has anti metal oxide properties [136] and has been previously used to etch $\mathrm{ZnO}$ in this manner [71]. However, there were concerns that $\mathrm{H}_{3} \mathrm{PO}_{4}$ may react with the $\mathrm{ZnO}$ surface and bind to the surface in a similar manner to phosphonic acid [137]. Therefore, hydrochloric acid ( $\mathrm{HCl}$, also used in [133]) was also trialled in order to test whether any chemical adhesion affected the growth of the nanorods. Both acids were prepared so that they have the same pH, 3.05 [62] Any difference in nanorod growth between samples etched in the different acids can then be attributed to chemical interactions, rather than differences in acidity.

In order to observe the effectiveness of the acid in etching the $\mathrm{ZnO}$ layer, a number of samples were prepared for observation in the SEM. Substrates were imme-
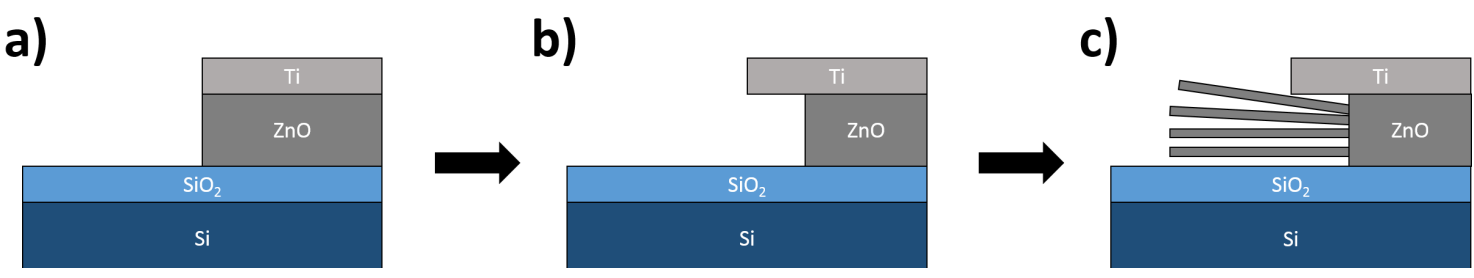

Figure 5.3: a) A schematic of the cross section of the sputtered layers before an acid etch. b) Acid etches the $\mathrm{ZnO}$ layer leaving an overhang of the Ti layer. c) During hydrothermal growth the overhang constrains the nanowires to lateral growth (in contrast to figure 5.2b). Not to scale. 
rsed in acid for a time ranging from 1 to 30 seconds and then immediately rinsed in DI water. These etched substrates were cleaved and then mounted such that the cross-section of the $\mathrm{ZnO} / \mathrm{Ti}$ stack could be imaged in the SEM.

The extent of the acid etch and the overhanging Ti layer is apparent in figure 5.4 and the depth to which the $\mathrm{ZnO}$ is etched, as a function of time, is plotted in figure 5.5. It is apparent that both acids etch at effectively the same rate of roughly 25 nm per second.

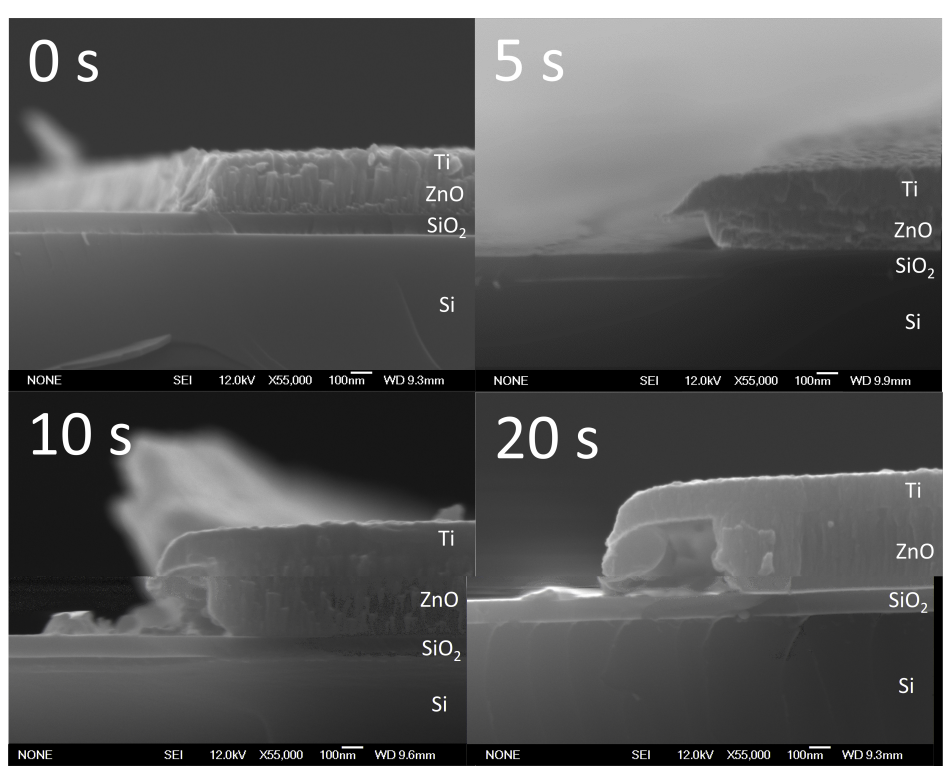

Figure 5.4: SEM micrographs of samples etched in phosphoric acid for the labelled times. The Overhanging Ti layer is clearly visible.

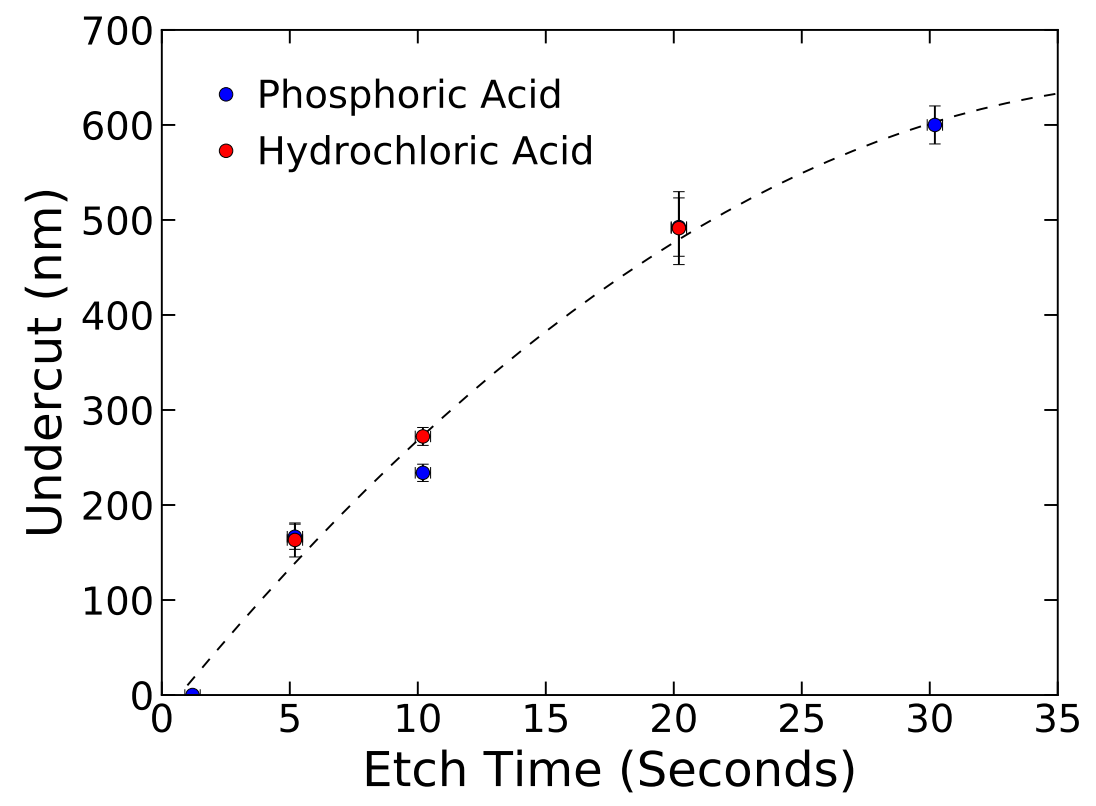

Figure 5.5: The etch rate for $\mathrm{H}_{3} \mathrm{PO}_{4}$ and $\mathrm{HCl}$. Both acids etch at effectively the same rate. The dashed line is an arbitrary curve fit. 


\subsubsection{Wire morphology}

In order to observe the effect of the overhanging Ti layer on the nanorod structure, numerous samples were prepared and etched for different times in either $\mathrm{H}_{3} \mathrm{PO}_{4}$ or $\mathrm{HCl}$. Nanorods were then grown on these samples, using the hydrothermal growth procedure described in section 4.1.5.

After hydrothermal growth, the nanorods can be viewed under a microscope. The hemispherical nanorod structures have a relatively large height and due to the small depth of focus of the microscope, are not easily focussed. In contrast, the lateral growths, which have a very small vertical height, can be easily focused. This means the success of lateral growth can be easily observed under a microscope.

Figure 5.6 compares samples etched for varying times in $1 \mathrm{mM}$ phosphoric acid. Short etch times of a few seconds continued to produce vertical nanorods (figure 5.6a) and are indistinguishable (under the microscope) from unetched samples. Etch times of 20 seconds, however, produce a uniform lateral nanorod structure (figure 5.6b). If the etch time is extended to greater than 30 seconds, the nanorods become sparser and less regular (figure 5.6c). This is due to a collapse of the overhanging Ti layer, obscuring the $\mathrm{ZnO}$ seed layer during hydrothermal growth.

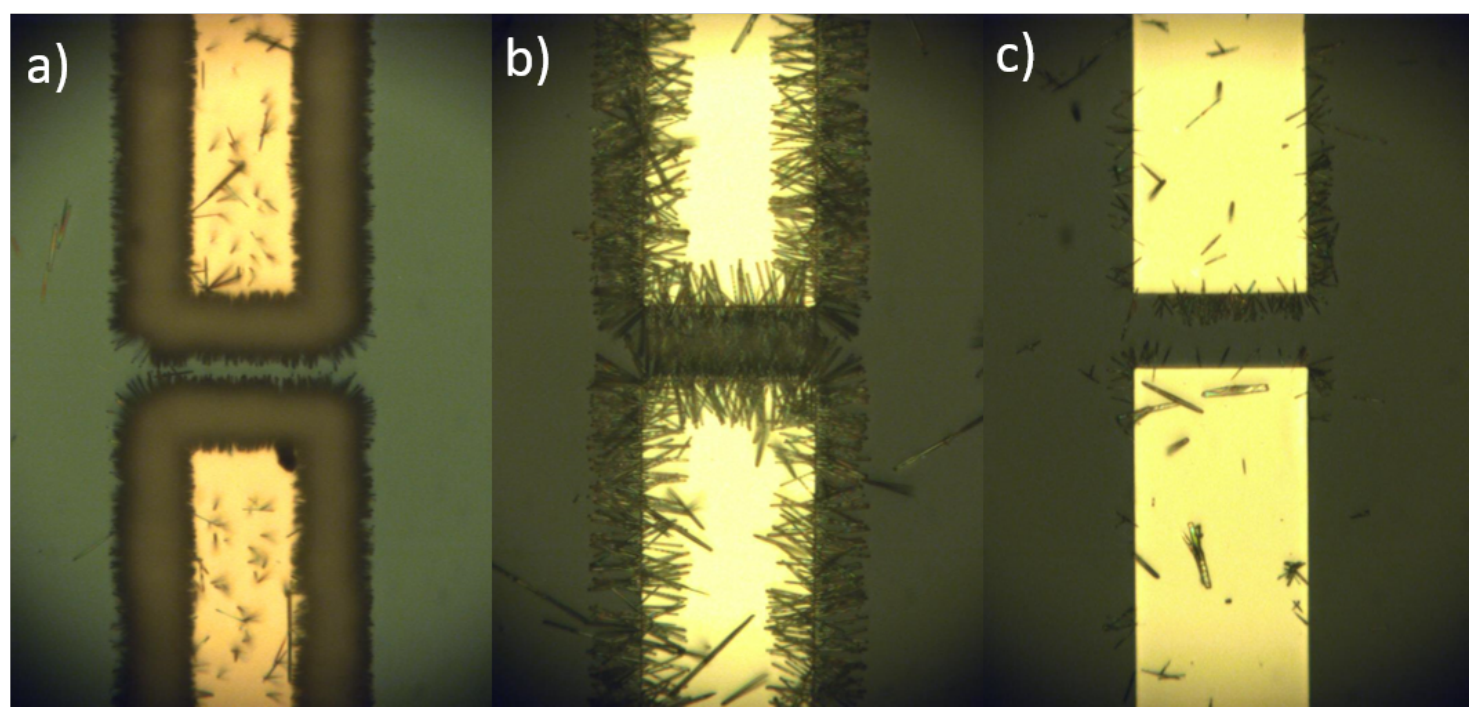

Figure 5.6: Representative microscope images of nanorods grown on substrates etched with phosphoric acid. The channels between the electrodes (yellow areas) are $40 \mu \mathrm{m}$. a) an etch of less than 10 seconds leads to a nanorod structure with a substantial vertical height (note the lack of focus). b) an etch of around 20 seconds leads to exclusive lateral nanorods. c) An etch longer than 30 seconds leads to an irregular and relatively sparse nanorod distribution. 
To confirm the exact shape of the nanorod structures, samples were cleaved and mounted such that the cross section of the sample can be viewed in the SEM. Figure 5.7 shows micrographs of samples that were etched in acid for times varying from 2.5 seconds to 30 seconds. As the etch time is increased, the nanorods become further constrained to the horizontal plane. The maximum angle of the nanorods relative to the horizontal as a function of etch time is shown in figure 5.8 .

A maximum theoretical possible angle can be calculated for a line drawn from the bottom of the $\mathrm{ZnO}$ layer to the bottom of the edge of the Ti overhanging layer. Assuming that the overhang is not damaged during growth and that the nanorods are infinitely thin, the maximum growth angle is given by:

$$
\theta=\tan ^{-1}\left(\frac{t}{d}\right)
$$

Where $d$ is the depth of the undercut (see figure 5.5) and $t$ is the thickness of the $\mathrm{ZnO}(200 \mathrm{~nm})$. The maximum theoretical angle is also plotted in figure 5.8 and is only just above the error bars of the measured values. This is to be expected, however, as the nanorods have a non-zero thickness which would further constrict the maximum possible angle. The similarity between the results and this fit, supports the idea that the Ti layer acts as a mechanical barrier to nanorod growth.

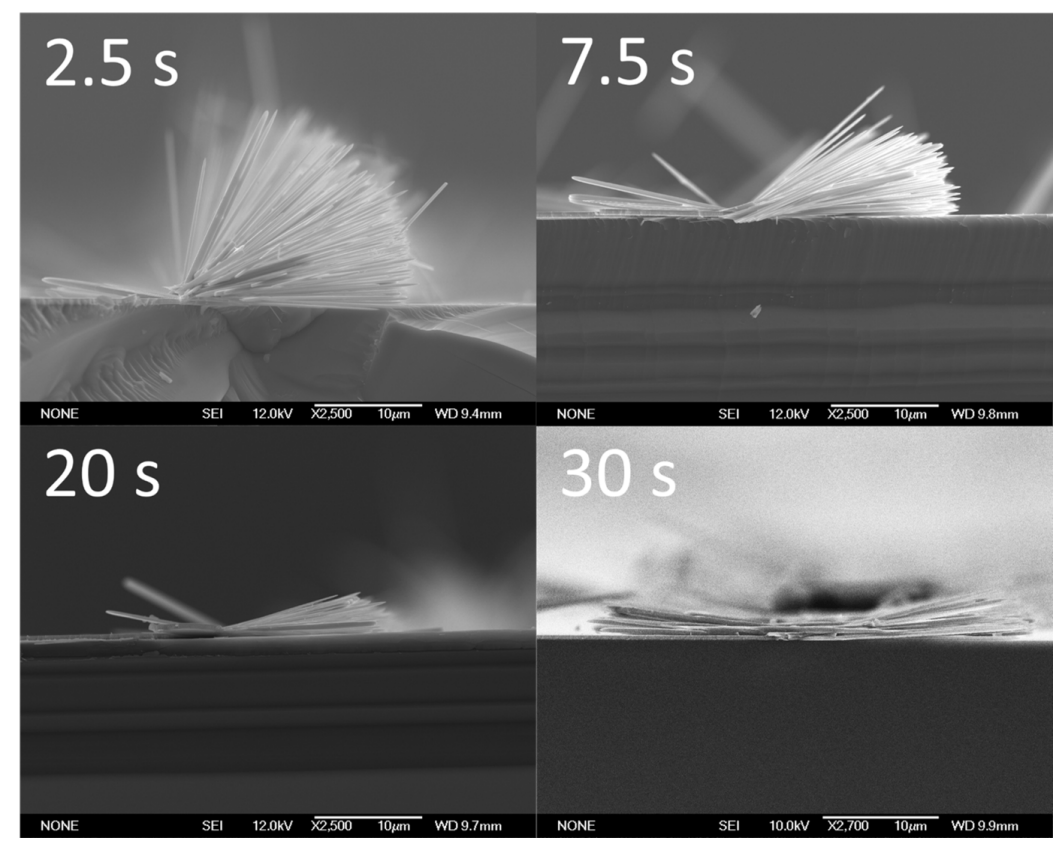

Figure 5.7: SEM micrographs of rod structures grown on substrates etched in $1 \mathrm{mM} \mathrm{H}_{3} \mathrm{PO}_{4}$ for the labelled times. Notice that the wire structure becomes progressively more constrained to the horizontal plane as etch time is increased. 


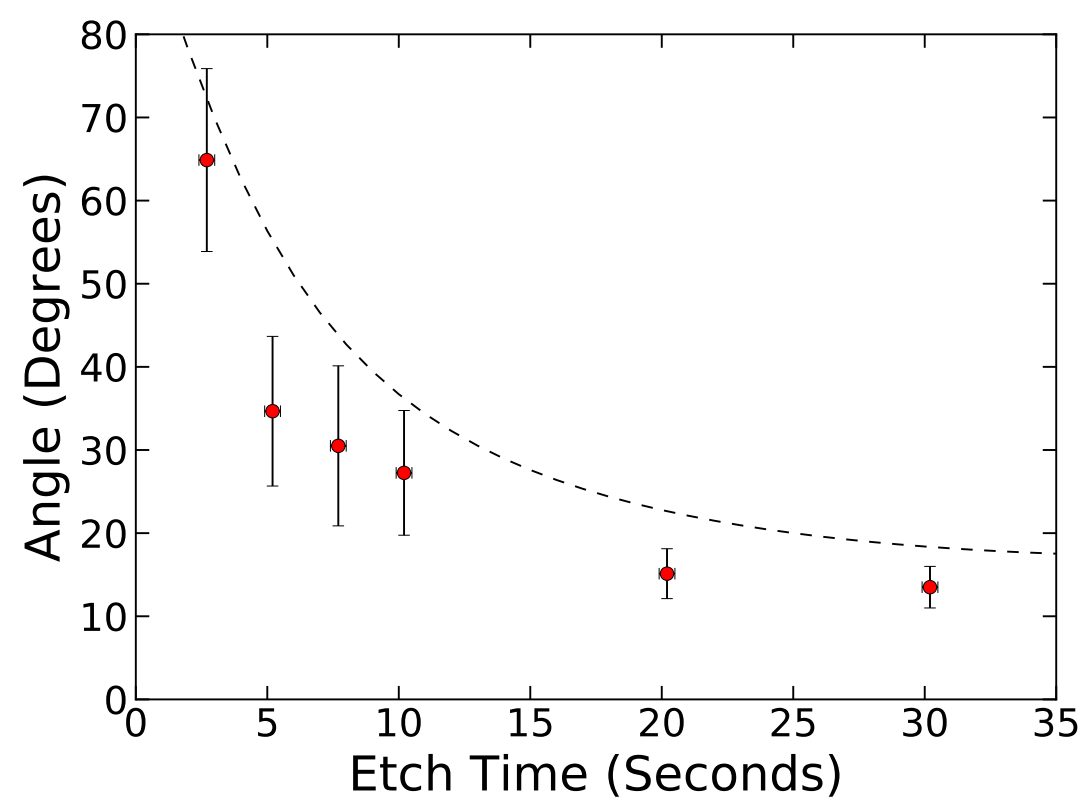

Figure 5.8: Maximum angle of nanorods relative to the horizontal as a function of etch time in acid. The curve describes the theoretical maximum possible angle assuming a rigid Ti overhang.

As in section 5.1.1, these growths were repeated with samples etched with 0.89 $\mathrm{mM} \mathrm{HCl}$ (same $\mathrm{pH}, 3.05$, as $1 \mathrm{mM} \mathrm{H}_{3} \mathrm{PO}_{4}$ [62]). The nanorod structures of samples etched in $\mathrm{HCl}$ showed no observable differences when compared to those etched in $\mathrm{H}_{3} \mathrm{PO}_{4}$. Therefore, it can be confirmed that there are no chemical interactions on the $\mathrm{ZnO}$ surface that affect hydrothermal growth.

It should be noted that the nanorods sometimes grow backwards over the $\mathrm{ZnO} / \mathrm{Ti}$ stack. The nanorods are thicker $(400 \pm 130 \mathrm{~nm})$ than the $\mathrm{ZnO} / \mathrm{Ti}$ stacks $(300 \mathrm{~nm})$. During hydrothermal growth the back face of a lateral nanorod can become exposed once its diameter grows larger than the thickness of the $\mathrm{ZnO} / \mathrm{Ti}$ stack. At this point the nanorod can start growing backwards over the $\mathrm{ZnO} / \mathrm{Ti}$ stack.

It is also interesting to note the differences in length of the nanorods for different etch times. This is most apparent in figure 5.6a and 5.6b. The channel is $40 \mu \mathrm{m}$ wide on both of these samples, however, the channel is only bridged by the lateral wire structure. This is not surprising as the lateral nanorod structures consist of fewer nanorods than the hemispherical structures. During hydrothermal growth there are a limited amount of ions available. This means that the lateral nanorod structure has more ions available per nanorod and hence they grow longer.

In summary, a 20 second etch of $1 \mathrm{mM} \mathrm{H}_{3} \mathrm{PO}_{4}$ or $0.89 \mathrm{mM} \mathrm{HCl}$ was found to be reliable and consistent at producing a lateral nanorod structure. The maximum angle at which the nanorods deviate from the horizontal is $15^{\circ} \pm 3^{\circ}$. Etch times of 30 seconds or more may be used to constrain the nanorods to a slightly smaller angle, but do not always produce a consistent nanorod structure as depicted in figure 5.6c. 


\subsection{ZnO Nanorod and Nanowire Device Structures}

Two types of devices, labelled nanorods and nanowires, were fabricated and tested. They are described in detail in sections 5.2.1 and 5.2.2.

\subsubsection{Nanorod Devices}

Substrates were prepared using the techniques described in section 4.1. Electrodes were patterned onto the substrates using photolithography and sputtered with $200 \mathrm{~nm}$ of $\mathrm{ZnO}$ and capped with $100 \mathrm{~nm}$ of Ti. The channel between the electrodes was $40 \mu \mathrm{m}$. A $25 \mathrm{mM}$ growth solution, with $8 \mathrm{mM} 800 \mathrm{gmol}^{-1}$ PEI, was preheated in a water bath at $95^{\circ} \mathrm{C}$ for one hour. The substrates are then added to the growth solution. After 19 hours the samples are removed, rinsed in DI water and dried with $\mathrm{N}_{2}$ gas.

This hydrothermal growth recipe was taken from previous work by Conor BurkeGovey $[44,100]$. It is optimised to produce "hierarchical nanowires" that consist of a large nanorod (some hundreds of nanometers in diameter) that then tapers into a nanowire (a few nanometres in diameter). The initial results presented in this section were unable to replicate this. Instead only nanorods are present, a typical example of which is depicted in figure 5.9. These nanorods have a diameter of $400 \pm 130 \mathrm{~nm}$ and a length of $22 \pm 0.4 \mu \mathrm{m}$.

Following hydrothermal growth, the samples are encapsulated with Su-8 photoresist to reduce leakage currents between the gate and source, although without the hardbaking step (see section 6.1.3). The exposed area across the channel is $30 \mu \mathrm{m}$ by $150 \mu \mathrm{m}$. A completed sample is depicted in figure 5.10 .

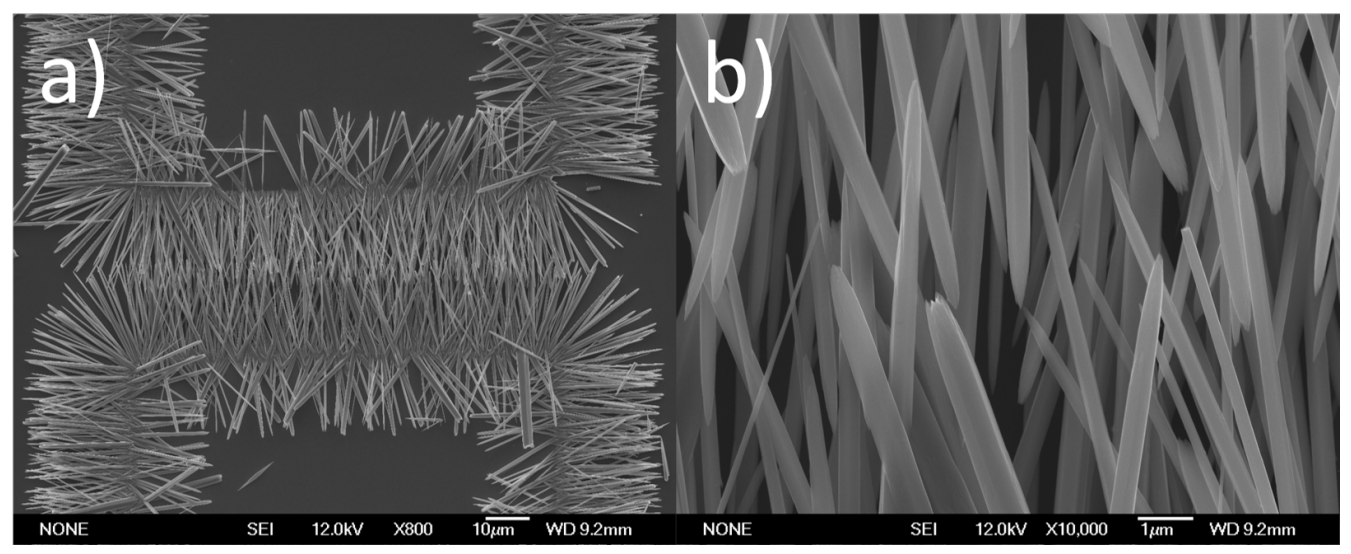

Figure 5.9: SEM micrographs of $\mathrm{ZnO}$ nanorods grown in a $25 \mathrm{mM}$ growth solution with $8 \mathrm{mM}$ PEI. The substrate was etched with $1 \mathrm{mM} \mathrm{H}_{3} \mathrm{PO}_{4}$ for 20 seconds. a) The channel between the electrodes is $40 \mu \mathrm{m}$ wide and bridged by nanorods. b) Magnification of the centre of the channel showing the intersection of the nanorods. 


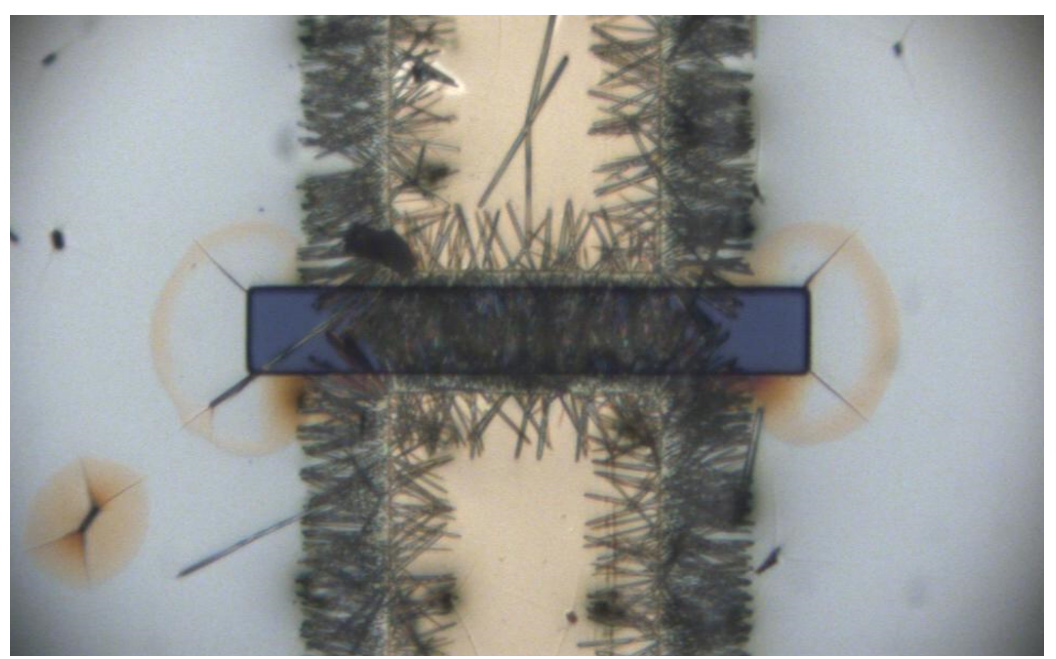

Figure 5.10: A nanorod sample. The channel is $40 \mu \mathrm{m}$ wide and the transparent Su-8 encapsulation layer has a $30 \mu \mathrm{m}$ by $150 \mu \mathrm{m}$ hole across the channel.

\subsubsection{Nanowire Devices}

The devices described in section 5.2.1 were fabricated using a hydrothermal growth solution which contained PEI supplied more than 18 months ago. It was determined that the PEI must have degraded and so a new bottle was obtained. Devices grown using the new PEI have a notably different nanostructure to those produced with the old PEI and consistent with the previous work in $[44,100]$.

The nanostructures of these new devices consist of primary nanorods (identical in both shape and size to those discussed in section 5.2.1) and a number of much smaller nanowires that have nucleated on the surface of the nanorods. Figure 5.11a shows an SEM micrograph of the hybrid nanorod/wire structure. The nanowires are much smaller than the nanorods, with a diameter of $20 \pm 5 \mathrm{~nm}$ (see figure 5.11b) and length of the order of $10 \mu \mathrm{m}$ long (It is difficult to identify the exact physical location where a nanowire begins and therefore more accurate measurement is not possible).

In order to electrically characterise the nanowires, devices were fabricated with an increased channel between electrodes of $60 \mu \mathrm{m}$ (compared to $40 \mu \mathrm{m}$ used for the nanorods samples in section 5.2.1). This ensures that the channel is not bridged by the nanorods and any current passing through the device must go through the nanowires (see figure Figure 5.12). The electrodes were defined with photoresist and sputtered with $200 \mathrm{~nm}$ of $\mathrm{ZnO}$ and $100 \mathrm{~nm}$ of Ti. The substrates are then etched in $1 \mathrm{mM} \mathrm{H}_{3} \mathrm{PO}_{4}$ for $20 \mathrm{~s}$ and a $25 \mathrm{mM}$ growth solution, with 8 $\mathrm{mM}$ of "new" PEI, is prepared. The growth solution is preheated for one hour at 
$95^{\circ} \mathrm{C}$ and then the samples are added for 19 hours. Finally samples were encapsulated with SU-8, leaving a $10 \mu \mathrm{m}$ by $150 \mu \mathrm{m}$ area exposed across the channel. This hole in the SU-8 only exposes the nanowires, while all nanorods are fully encapsulated. Figure 5.12 shows an optical image of a $\mathrm{ZnO}$ nanowire device.

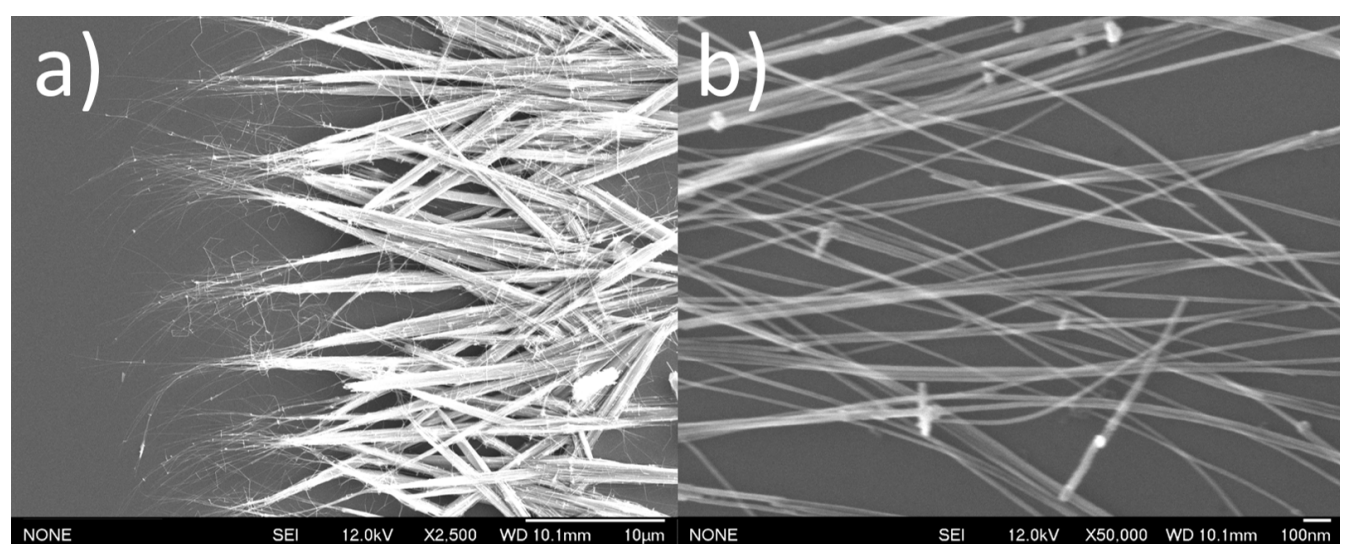

Figure 5.11: SEM micrographs of $\mathrm{ZnO}$ nanorods/nanowires grown in a $25 \mathrm{mM}$ growth solution with $8 \mathrm{mM}$ of "new" PEI. The substrate was etched with $1 \mathrm{mM} \mathrm{H}_{3} \mathrm{PO}_{4}$ for 20 seconds. a) The large rods have much much smaller nanowires growing off of them. b) The nanowires are $20 \pm$ $5 \mathrm{~nm}$ in diameter.

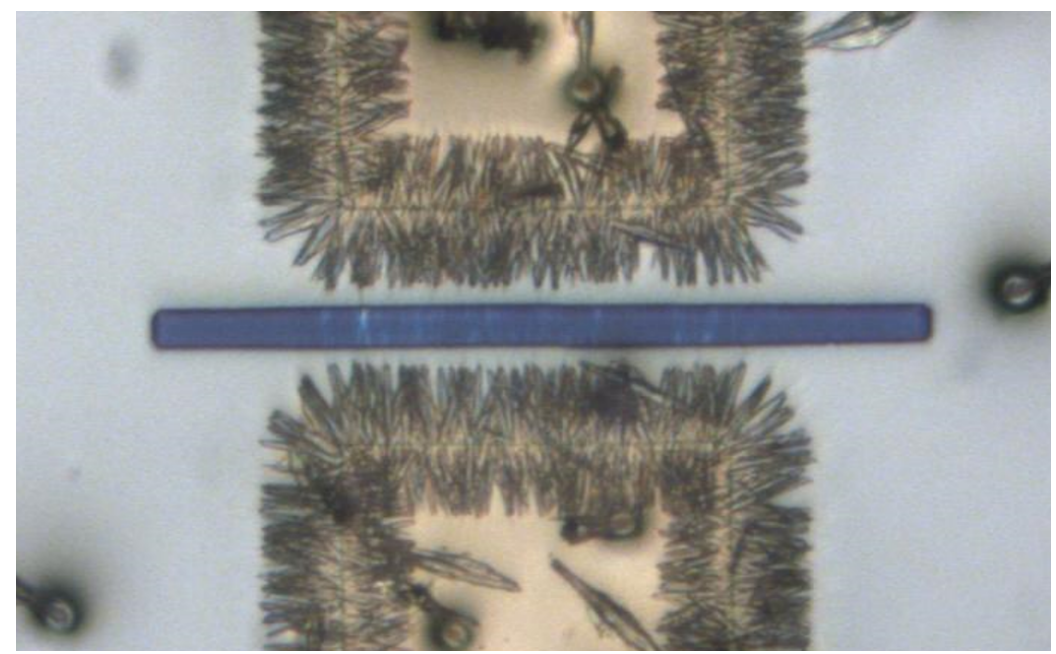

Figure 5.12: A nanowire sample. The channel is $60 \mu \mathrm{m}$ wide and the transparent Su-8 encapsulation layer has a $10 \mu \mathrm{m}$ by $150 \mu \mathrm{m}$ hole across the channel. The nanowires are only just visible as the white lines crossing the channel. 


\subsection{Conclusions}

In conclusion, the growth of exclusively lateral nanowires can be achieved using an acid etch of the $\mathrm{ZnO}$ seed layer in conjunction with a Ti capping layer. The $\mathrm{ZnO}$ seed layer can be etched with dilute acid $\left(1 \mathrm{mM} \mathrm{H}_{3} \mathrm{PO}_{4}\right.$ or $\left.0.89 \mathrm{mM} \mathrm{HCl}\right)$ at a rate of roughly $25 \mathrm{~nm}$ per second. The overhang of the Ti capping layer acts as a physical barrier to nanowire growth and the angular distribution of nanowires can be controlled with the etch time.

Two device types, nanorods and nanowires, were fabricated. The nanorods are fabricated using a hydrothermal growth recipe containing old PEI and are $400 \pm$ $130 \mathrm{~nm}$ in diameter and $22 \pm 0.4 \mu \mathrm{m}$ long. The nanowire samples were grown using the same recipe except that a new bottle of PEI was used. These samples consist of nanorods that have much thinner nanowires (of a diameter of only 20 $\pm 5 \mathrm{~nm}$ ) growing off them.

The variation in $\mathrm{ZnO}$ nanostructures as a result of the degradation of PEI may be of interest in understanding the role PEI has in hydrothermal growth. As described in section 2.2.3, the mechanism by which PEI influences the shape of the $\mathrm{ZnO}$ nanostructures is a complicated combination of steric hindrance and mediation of ion concentration. The growth of nanowires only occurs with the new PEI, while the nanorods are effectively identical between growths with either new or old PEI. This seems to imply that there is some effect that is only present in the new PEI that leads to nanowire formation. It is possible that the formation of the nanowires occurs under a regime of low ion concentration, late in the hydrothermal growth. This could be caused by the gradual release of chelated $\mathrm{Zn}^{2+}$ ions from the PEI molecules. If this is the case, then there must be some structural difference between the old and new PEI that influences the polymers' affinity for $\mathrm{Zn}^{2+}$. Further investigation into this mechanism and the degradation of PEI is beyond the scope of this thesis. 


\section{Chapter 6}

\section{Electrical Characterisation of ZnO Nanorod and Nanowire Devices}

In this chapter the nanorod and nanowire devices are electrically tested in order to determine their viability as an FET. This involves measuring their field dependence when dry and in liquid conditions. When immersed in liquid the device can be either backgated or topgated with a $\mathrm{Ag} / \mathrm{AgCl}$ electrode. Further descriptions of these setups are given in section 4.3.

\subsection{Nanorod Electrical Characterisation}

\subsubsection{Dry}

Samples may be gated through the $\mathrm{Si}$ substrate, with the $\mathrm{SiO}_{2}$ layer acting as a dielectric. A transfer curve of a backgated nanorod sample is shown in figure 6.1. The samples are found to be conductive and show negligible field dependence in the range of -10 to $10 \mathrm{~V}_{G}$. The maximum variation in drain-source current for a single sweep in figure 6.1 is $7 \%$. The nanorods effectively behave as an ohmic resistor (i.e. the drain-source current is proportional to the drain-source voltage) and are therefore not a viable material for an FET in this regime.

$\mathrm{ZnO}$ nanowires often require annealing at high temperatures of around 200 to 600 ${ }^{\circ} \mathrm{C}[66-69,72,75,78-80]$ in order to achieve field dependence. The as grown wires are highly conductive due to high dopant densities [78] and the anneal reduces the charge carrier concentration. The lack of field dependence of the nanorods in figure 6.1 is therefore unsurprising and likely due to high carrier concentrations in the surface of the nanorods.

\subsubsection{Liquid Gated (DI Water)}

For this measurement a PDMS well is placed over the sample and filled with DI water. Electrolysis of water occurs at $1.23 \mathrm{~V}$ [138] and this may interfere with the electrical measurements. To avoid this, all voltages are limited to $1 \mathrm{~V}$.

Figure 6.2 shows the transfer curve of the same device as figure 6.1. This measurement still involves gating through the $\mathrm{Si} / \mathrm{SiO}_{2}$ substrate, but with the device 

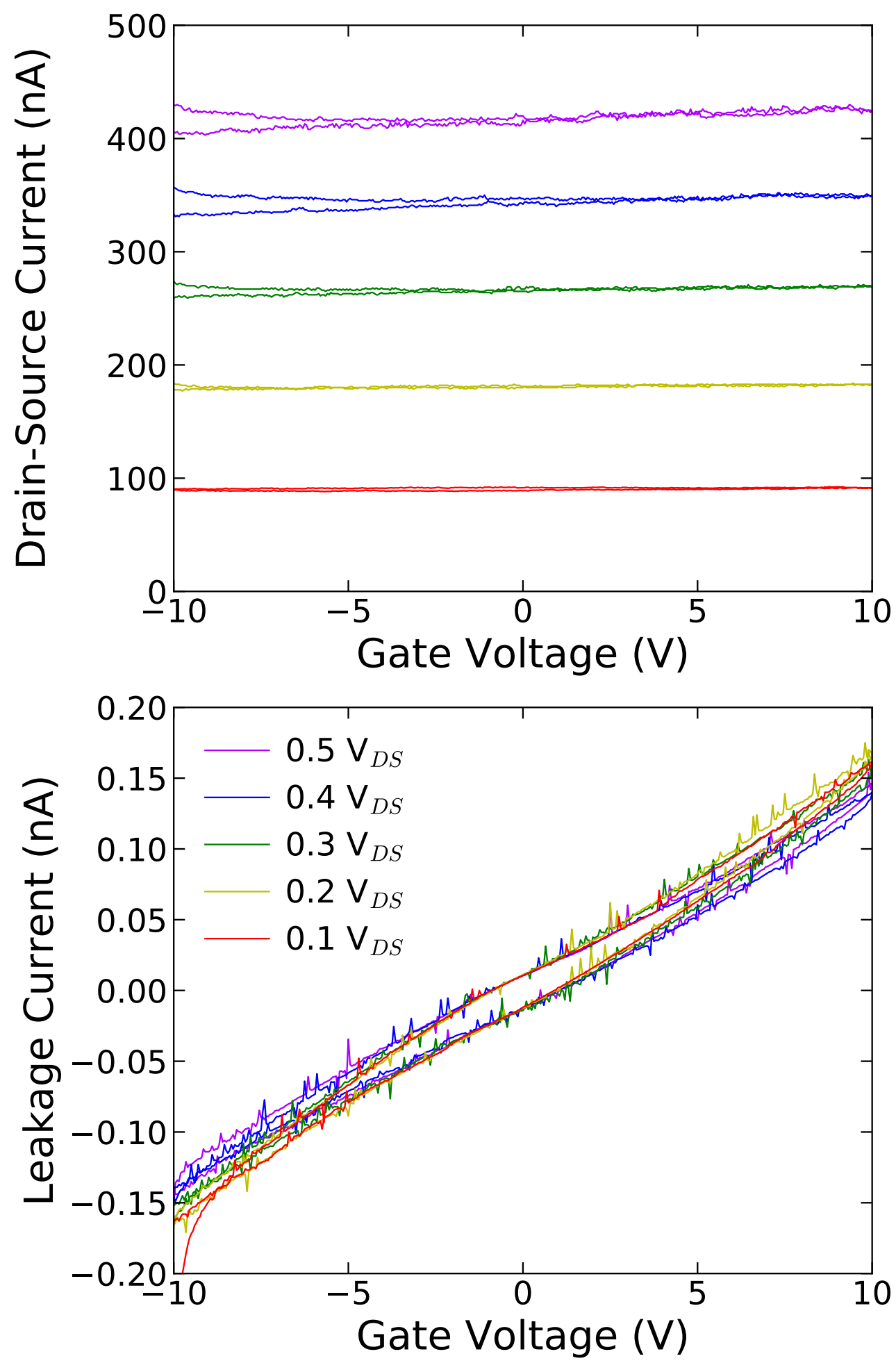

Figure 6.1: Transfer curve of a $\mathrm{ZnO}$ nanorod FET with (top) the drain-source current and (bottom) the leakage current. The gate voltage is swept from $-10 \mathrm{~V}$ to $10 \mathrm{~V}$ and back to $-10 \mathrm{~V}$ for the listed drain-source voltages (legend applies to both plots). There is no substantial field dependence over this range of gate voltages. 
submerged in DI water. The nanorod device shows a clear field dependence with a maximum on/off ratio of $10^{3}$ across the gate voltage range of $\pm 1 \mathrm{~V}$. There is a clear hysteresis and the threshold voltage differs between the the forwards sweep, at $0.02 \mathrm{~V}$, and the backwards sweep, at $0.39 \mathrm{~V}$. The maximum transconductance is $250 \mathrm{nS}$ and the minimum subthreshold swing is $183 \mathrm{mV} /$ decade.

In this setup, the channel of the device is completely immersed in DI water and an electric double layer can form around the nanorods. As described in section 3.1.2, the electric double layer can produce a very large capacitance (several $\mu \mathrm{Fcm}^{-2}$ $[57,60])$. It would seem that the use of DI water is strong enough to deplete the $\mathrm{ZnO}$ nanorods and achieve an off state, unlike in the dry setup.

The devcie can also be measured in the topgate configuration. This setup involves a Ag/ AgCl electrode being inserted into the DI water held within the PDMS well. The gate voltage is applied to this electrode.

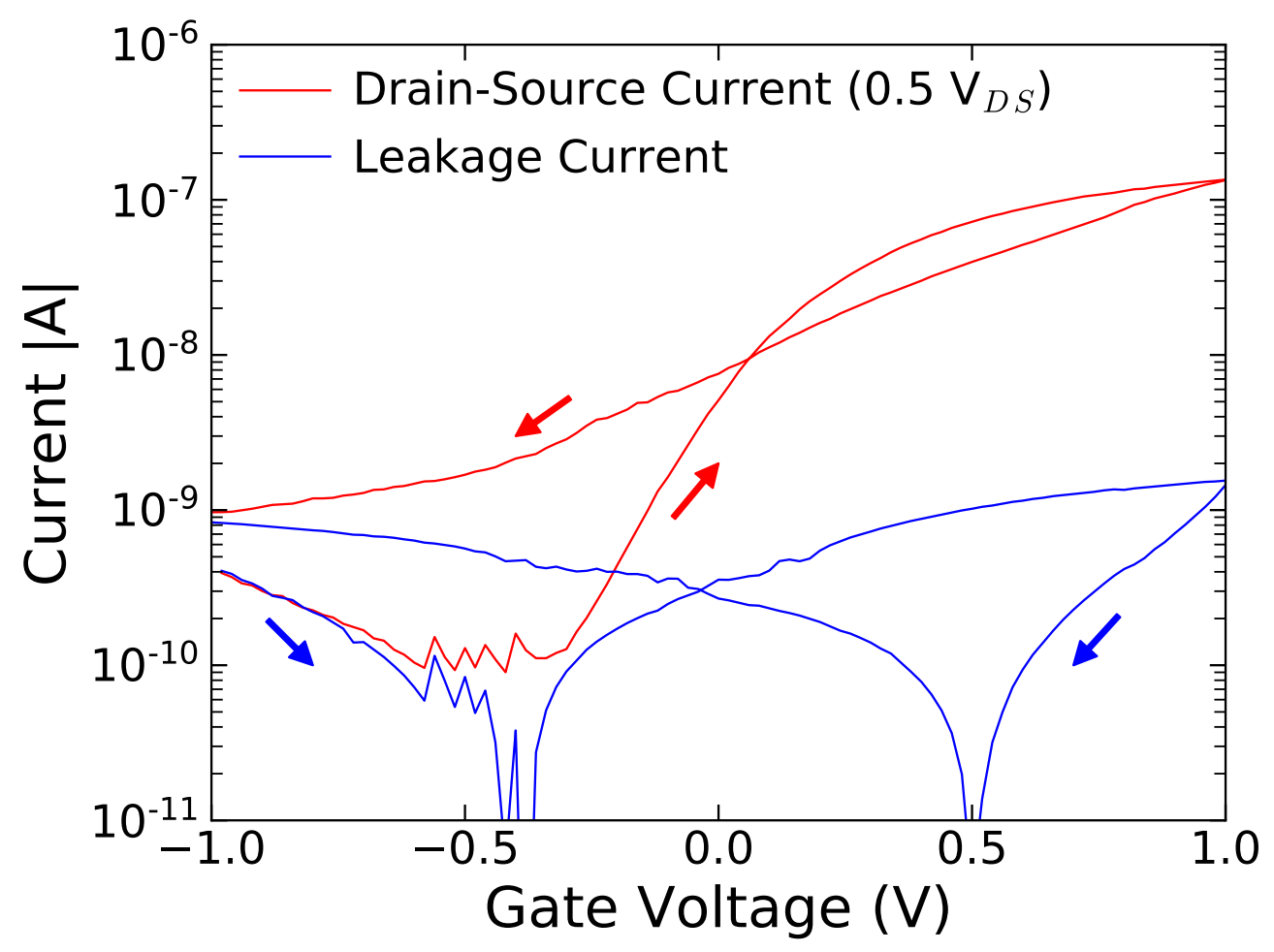

Figure 6.2: Transfer curve of a backgated ZnO nanorod FET with DI water on top. There is clearly a substantial field dependence with an on/off ratio of approximately $10^{3}$. Note that the absolute value of the respective currents are plotted. 
The topgated transfer curve of the same device (as in figure 6.1 and 6.2) is given in figure 6.3. The behaviour is very similar to when the device is gated through the $\mathrm{Si} / \mathrm{SiO}_{2}$ as in section 6.1.2. The maximum on/off ratio is still approximately $10^{3}$, but now the device exhibits a much larger hysteresis. The threshold voltage is larger than the backgated setup at $0.47 \mathrm{~V}$ for the forward sweep and $0.25 \mathrm{~V}$ for the backward sweep. The device also switches on much faster with a maximum transconductance of $430 \mathrm{nS}$ and minimum subthreshold swing of $87 \mathrm{mV}$ /decade.

The topgated configuration, using the $\mathrm{Ag} / \mathrm{AgCl}$ electrode, produces a larger threshold voltage for the forwards sweep, $0.47 \mathrm{~V}$, than when backgated through the $\mathrm{Si} / \mathrm{SiO}_{2}$ substrate. A shift in threshold voltage is not unexpected as the physical gate setup is different. In the backgated configuration there will be a coupling between the $\mathrm{SiO}_{2}$ layer and the DI water. This is not the case for the topgated configuration and the positive shift can be attributed to a larger potential barrier between the $\mathrm{ZnO}$ and the $\mathrm{Ag} / \mathrm{AgCl}$ electrode.

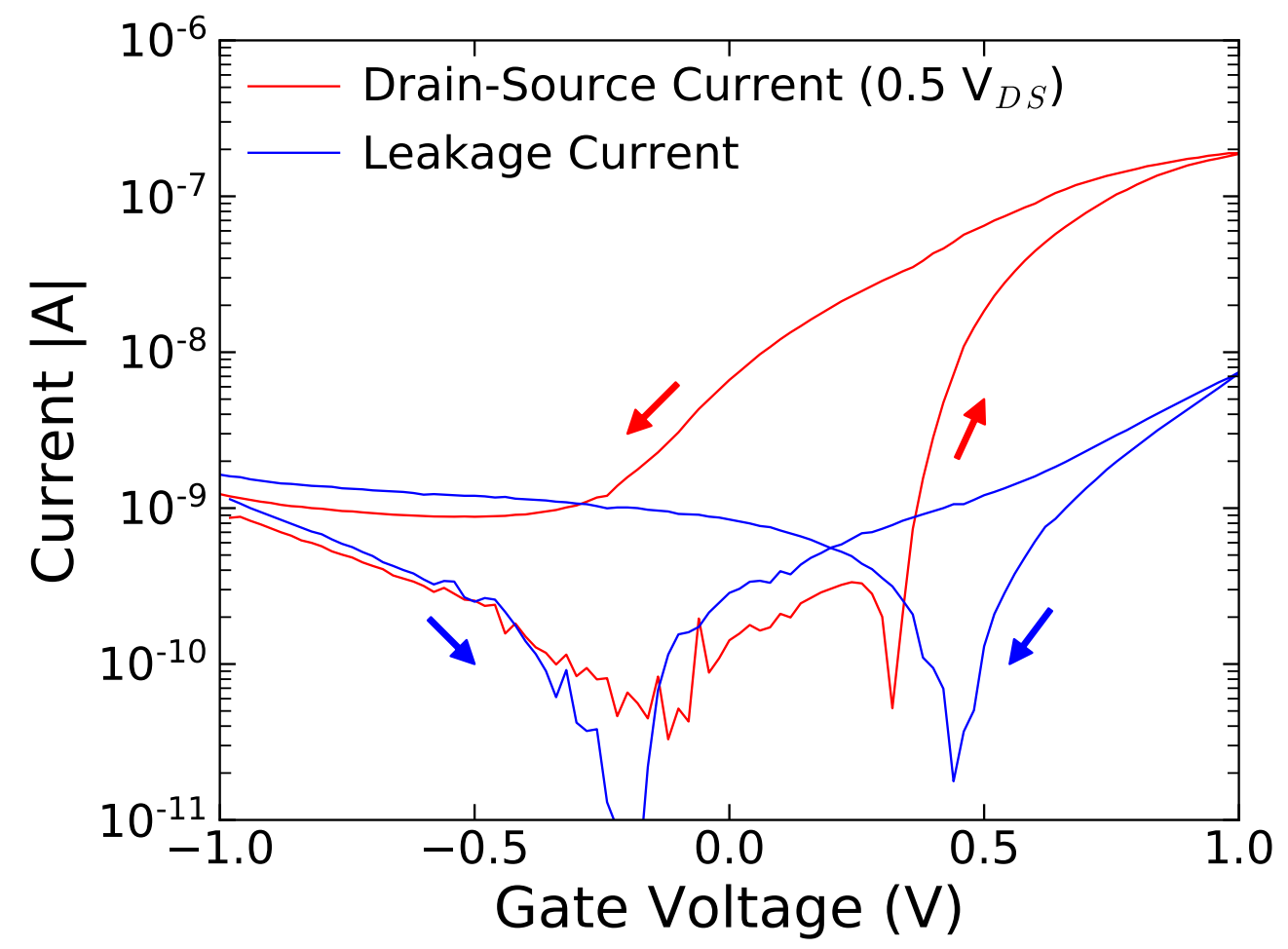

Figure 6.3: Transfer curve of $\mathrm{ZnO}$ nanorod FET gated through a $\mathrm{Ag} / \mathrm{AgCl}$ electrode in DI water. The on/off ratio is approximately the same $\left(10^{3}\right)$ as in the backgated configuration. The threshold voltage, however, has shifted to more positive voltages and switches much faster. 


\subsubsection{Hardbaking SU-8 and Nanorods}

The purpose of the SU-8 encapsulation layer in these devices is to reduce leakage currents that occur through contact between the DI water and the electrodes. Figure 6.4 shows the transfer curve of a topgated nanorod device in DI water (with its respective leakage current $\left(\mathrm{I}_{G}\right)$ ), before encapsulation. The leakage current is clearly comparable in magnitude to the drain-source current. At negative gate voltages, the leakage current is interfering with and dominating the current reading for the drain-source current. Consequently an encapsulation layer is necessary to insulate the gate. This is the case for the device measured in section 6.1.2 where the leakage current is approximately two orders of magnitude smaller.

SU-8 can be hardbaked $\left(200{ }^{\circ} \mathrm{C}\right.$ for 15 minutes, see section 4.1 .7$)$ making it extremely resistant to organic solvents. This was not necessary for the liquidgated measurements in section 6.1.2, but will be necessary for the GOPS functionalisation in chapter 7. Figure 6.4 shows the drain-source current of the nanorod device after it been hardbaked (The device was baked without SU-8 to demonstrate that the hardbaking of the nanorods, not the Su-8, is causing the change in conductivity). The device is substantially more conductive by at least two orders of magnitude. Furthermore the field dependence of the device is much smaller with an on/off ratio of about two.

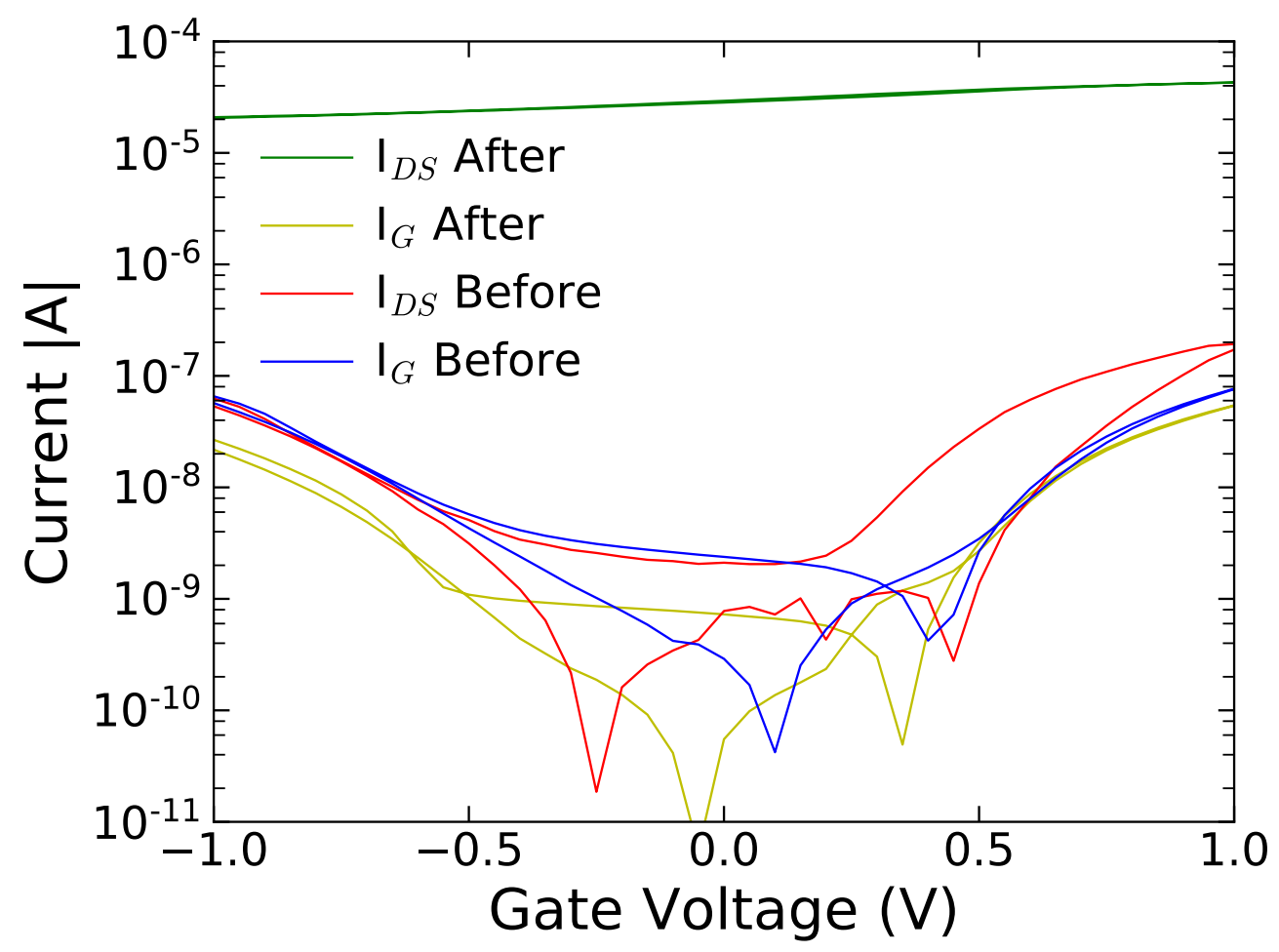

Figure 6.4: Transfer curve of a topgated nanorod device in DI water, before and after baking at $200{ }^{\circ} \mathrm{C}$ for 15 mins. Before baking the device has very large leakage currents. After the hardbake, however, it is highly conductive and shows minimal field dependence. 
$\mathrm{ZnO}$ nanowire devices often require annealing of the nanowires at temperatures of around 200 to $600{ }^{\circ} \mathrm{C}[66-69,72,75,78-80]$ in order to achieve field dependence. This results in a reduction in the carrier concentration and the density of dopants in the nanowires $[69,78]$. The results of figure 6.4 contradict this and suggest an increase in carrier concentration. Further study would be necessary to identify the mechanism behind this effect. Energy-dispersive X-ray spectroscopy measurements, for example, might be a useful tool to identify whether, and in what way, the baking step is inducing changes in the doping level of the $\mathrm{ZnO}$ (e.g. desorption of $\mathrm{O}_{2}$ or adsorption of $\mathrm{H}_{2} \mathrm{O}$ ). Alternatively X-ray diffraction could be used to investigate changes in the crystallinity of the nanorods.

\subsection{Nanowire Electrical Characterisation}

In the same manner as the nanorod devices, the nanowires will be characterised as FETs in both dry and liquid conditions. The nanowire devices prove to have better transfer characteristics, with an on/off ratio about an order of magnitude larger than the nanorods, and is the preferred device for biosensing. Measurements are also made with the nanowires gated in tris buffer as many biosensing applications require operation in biological fluids.

\subsubsection{Dry}

The transfer curve for a nanowire sample, gated through the $\mathrm{SiO}_{2}$ substrate layer, is given in figure 6.5. The device is driven with a $\mathrm{V}_{D S}$ of $0.5 \mathrm{~V}$ and $\mathrm{V}_{G}$ sweeps from $-10 \mathrm{~V}$ to $10 \mathrm{~V}$ and back.

In contrast to the nanorod samples, the nanowires show a clear field dependence when dry. There is a distinctive on and off regime and the device measured in figure 6.5 has an on/off ratio of $3.3 \times 10^{4}$. There is negligible hysteresis and so only a single threshold voltage of $-0.8 \mathrm{~V}$, can be identified. The minimum subthreshold swing of this device is $450 \mathrm{mV} /$ decade and it has a maximum transconductance of $40 \mathrm{nS}$.

The nanowires are much thinner than the nanorods and are in very good contact with the $\mathrm{SiO}_{2}$ substrate. This is likely to be a major factor that contributes to the field dependence of these devices. It should be noted, however, that the origin of this field dependence may also be related to the crystallinity of the nanowires, or the exact chemical composition/doping level. Further investigation would be required to identify the relevance of these factors.

It should be noted, however, that there is a degree of variation between devices. In general these nanowire devices have a threshold voltage in the range of -2 to 2 $\mathrm{V}$ and on/off ratios generally vary between $10^{3}$ and $10^{5}$. The variation between 
devices is not completely surprising. The nanowire structures can be observed using an optical microscope and are often irregular in shape and density (see figure 5.12). This physical variation is no doubt a factor contributing to the electrical variation. Most obviously, the on current will be directly linked to the number of wires that bridge the electrodes of the device.

Some devices had $\mathrm{ZnO}$ nanorods or other large $\mathrm{ZnO}$ debris that cross the channel between electrodes. These devices still showed some field dependence (on/off ratios of about 10), but typically had very high off currents. As shown in section 6.1.1 the nanorods are conductive, but do not exhibit any field dependence when dry. The nanowires still achieve switching between on and off states, but the off state is limited by the relatively large, constant current through the debris. These devices do not exhibit characteristics representative of the nanowires. All nanowire devices were carefully inspected before testing to ensure that devices with debris present in the channel were identified and rejected. Further ingratiation into the reproducibility of transfer characteristics is covered in appendix A.

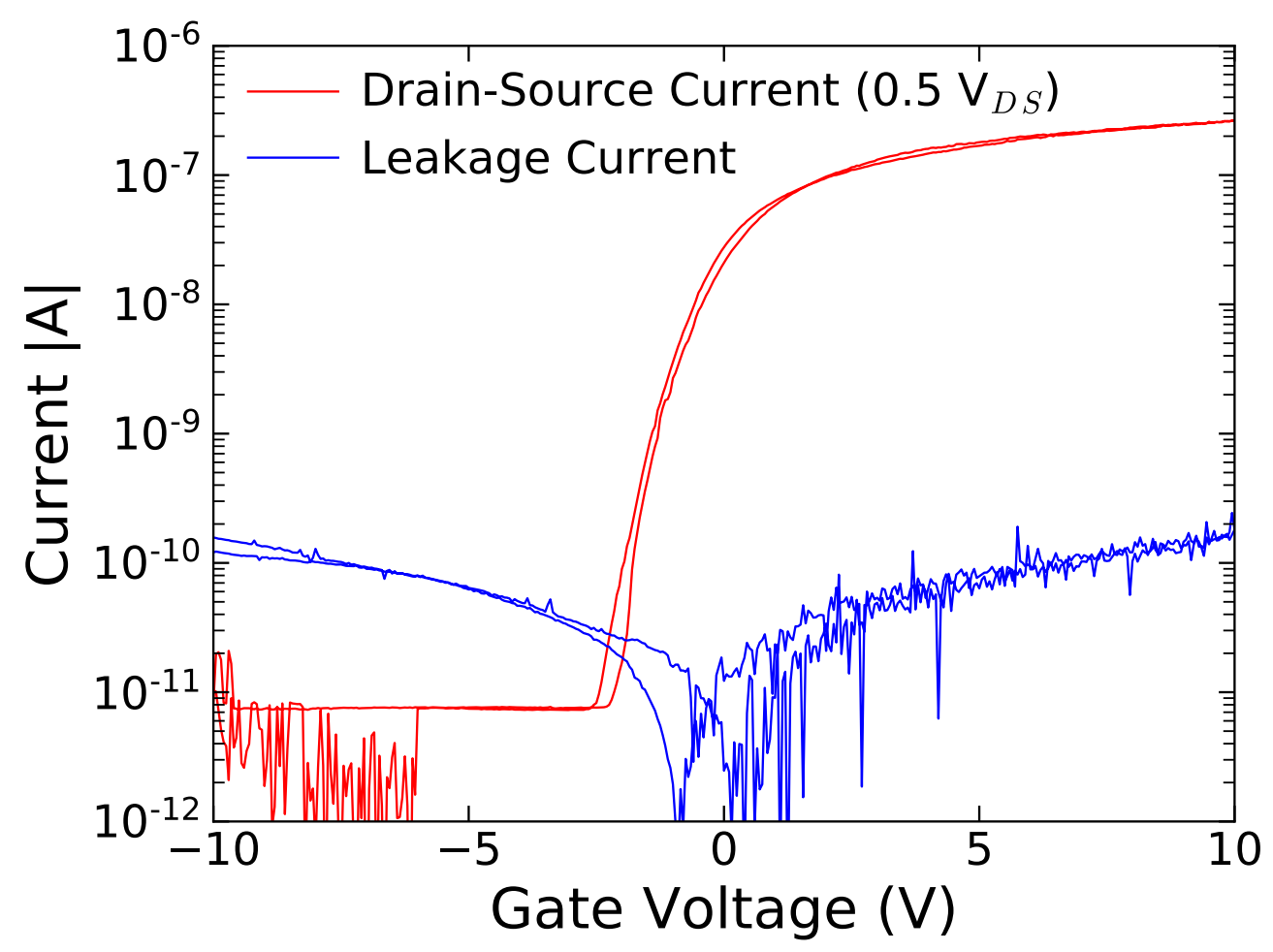

Figure 6.5: Transfer curve of a nanowire device gated through the $\mathrm{Si} / \mathrm{SiO}_{2}$ substrate. These devices show a clear field dependence with an on/off ratio of $3.3 \times 10^{4}$ and negligible hysteresis. 


\subsubsection{Liquid Gated (DI Water)}

It follows that the nanowire devices should be tested to see whether they are still viable FETs in liquid conditions. The transfer curve of a backgated nanowire device, with a PDMS well of DI water on top, is given in figure 6.6. As with the nanorod samples all volatges are limited to $\pm 1 \mathrm{~V}$ to avoid any effects of electrolysis.

The nanowire device exhibits clear switching within the range of -1 to $+1 \mathrm{~V}_{G}$. It would appear that this device does not quite reach saturation at $+1 \mathrm{~V}_{G}$, however, an on/off ratio of $7.5 \times 10^{4}$ is still achieved. Some hysteresis is clearly apparent and this device has a forward threshold voltage of $0.3 \mathrm{~V}$ and a backward threshold voltage of $0.5 \mathrm{~V}$. The minimum subthreshold swing is $78 \mathrm{mV} /$ decade and the maximum transconductance is $180 \mathrm{nS}$.

The switching in DI water occurs much faster than when dry. Switching of liquid gated $\mathrm{ZnO}$ FETs is often observed at gate voltages within the range of $\pm 3 \mathrm{~V}_{G}$ $[57,60,77,139]$, which is much closer to zero and over a narrower range than many dry FETs $[66,67,72-76,80]$. The increased switching speed when wet has also been observed in carbon nanotubes [82]. This is attributed to the smaller size and larger capacitance of the electric double layer in comparison to the $\mathrm{SiO}_{2}$ layer [57,82] (see section 3.1.2).

The on/ off ratio of the nanowire devices is larger than that of the nanorod devices and this is mainly attributed to the magnitude of the off current. At negative gate voltages, the nanowires achieve an off state where the current is substantially lower $\left(\approx 10^{-12} \mathrm{~A}\right)$ than that of the rods $\left(\approx 10^{-10} \mathrm{~A}\right)$. This could be attributed to the fact that the nanorod devices have an overall lower resistance (due to the larger cross section), or alternatively the centre of the nanorods might not be fully depleted. As described in section 3.1, the field effect induces band bending (and hence the change in conductivity) at the surface of the wires. Therefore, the strength of the band bending at the centre of the nanorods is not as strong as at the centre of the nanowires, due to their larger diameter. This results in a larger off current.

The same sample as measured in the backgated setup (section 6.2.2) can then be topgated using a $\mathrm{Ag} / \mathrm{AgCl}$ electrode and this is shown in figure 6.7. In this configuration, however, there is negligible hysteresis. There has also been a shift in threshold voltage to a more positive value $0.7 \mathrm{~V}$ (as was the case with the nanorod devices). The minimum subthreshold swing and the maximum transconductnace are $84 \mathrm{mV} /$ decade and $210 \mathrm{nS}$ respectively, while the on/off ratio is approximately $5 \times 10^{4}$. These results are effectively identical to the backgated (DI water) measurement (figure 6.6), except for the positive shift in the threshold voltage which can be attributed to the fact that there is no longer a coupling between the $\mathrm{SiO}_{2}$ layer and the DI water. 


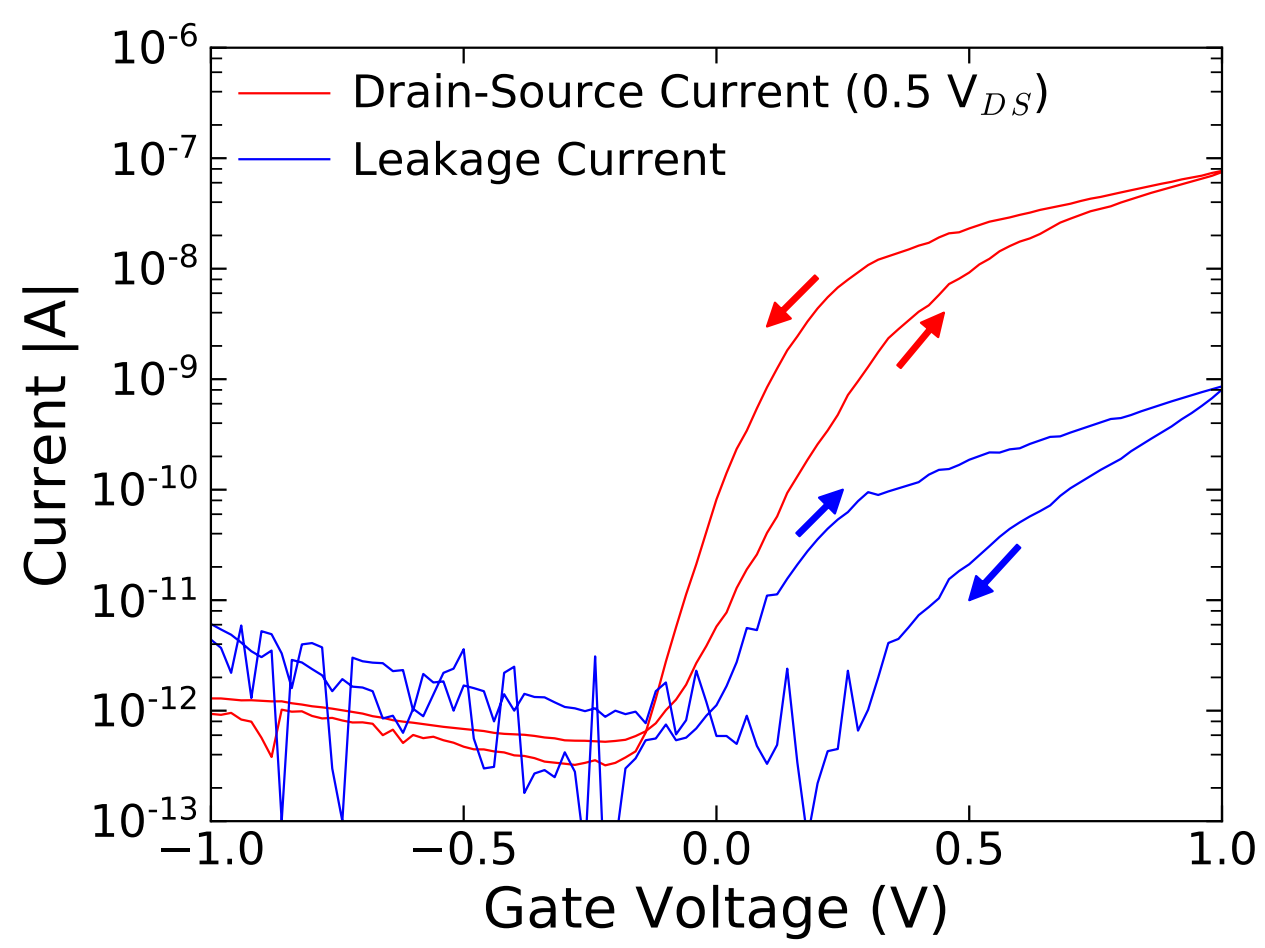

Figure 6.6: Transfer curve of a nanowire device gated through the $\mathrm{Si} / \mathrm{SiO}_{2}$ substrate while the nanowires are exposed to DI water. The device is able to switch across a much smaller $\mathrm{V}_{G}$ range while wet.

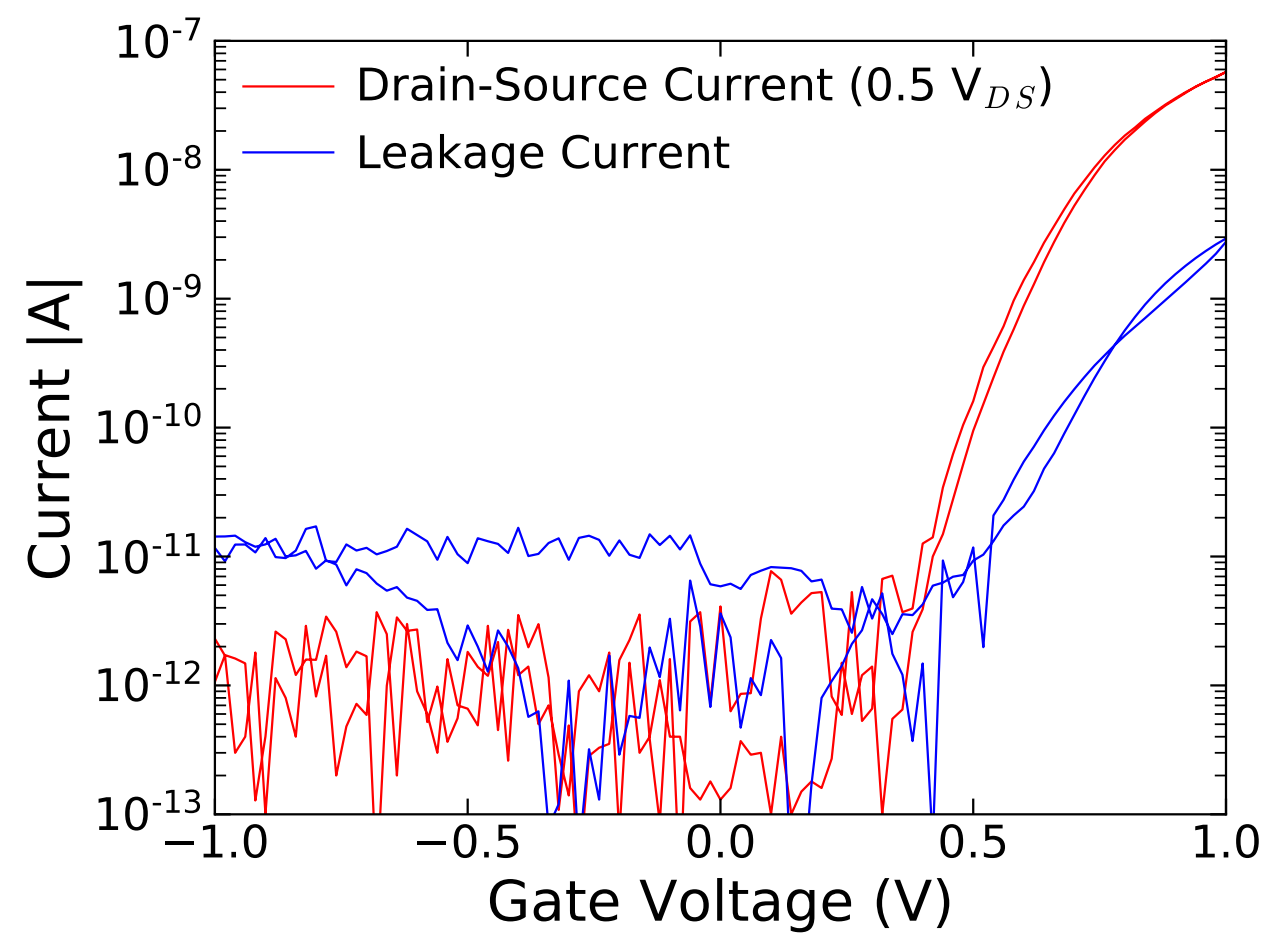

Figure 6.7: Transfer curve of a nanowire device topgated in DI using a $\mathrm{Ag} / \mathrm{AgCl}$ electrode. There is a positive shift in threshold voltage in comparison to the backgated (DI water) configuration. 


\subsubsection{Liquid Gated (Tris Buffer)}

The nanowire devices have shown better on/off ratios the nanorod devices and are the preferred platform for biosensing. Many biosensing applications require the detection of target molecules in biological fluids. Therefore, the nanowires devices will also be characterised in a buffer solution that replicates biological conditions. Tris(hydroxymethyl)aminomethane (tris) buffer is used as it falls in a $\mathrm{pH}$ range (7-9) that replicates physiological conditions [140].

$2 \mathrm{mM}$ Tris buffer is prepared by combining $0.04728 \mathrm{~g}$ trizma hydrochloride (Sigma Aldrich) with $200 \mathrm{ml}$ of DI water. The tris buffer is pippetted into a PDMS well on top of a nanowire sample as per the usual liquid gated setup (see section 4.3.2).

A transfer curve of a nanowire device in tris buffer, using the $\mathrm{Si} / \mathrm{SiO}_{2}$ substrate as the backgate is show in figure 6.8. The nanowire device exhibits clear switching within the range of -1 to $+1 \mathrm{~V}_{G}$, however the on/off ratio is particularly small at only 75. Furthermore there is substantial leakage of as much as $2.5 \mathrm{nA}$. The device has a forward threshold voltage of $0.11 \mathrm{~V}$ and a backward threshold voltage of $0.58 \mathrm{~V}$. The minimum subthreshold swing is $341 \mathrm{mV} /$ decade and the maximum transconductance is $5 \mathrm{nS}$.

When topgated using the $\mathrm{Ag} / \mathrm{AgCl}$ electrode immersed in the tris buffer, the same nanowire device as in figure 6.8 has a better on/off ratio of 390 in the range of -1 to $+1 \mathrm{~V}_{G}$. The device, however, does not achieve a full on state (ie. saturation). This is shown in the transfer curve in figure 6.9. The leakage is also still substantial and peaks at about $10 \mathrm{nA}$.

The minimum subthreshold swing is only slightly larger at $400 \mathrm{mV}$ /decade, but the maximum transconductance is much larger at $44 \mathrm{nS}$. The threshold voltage for the forward sweep is $0.50 \mathrm{~V}$ and $0.44 \mathrm{~V}$ for the backward sweep.

These measurements show that the nanowire devices can be used as FETs using tris buffer as a liquid dielectric. Switching is obtainable within a narrow gate voltage range ( -1 to $+1 \mathrm{~V}_{G}$ ). The FET parameters measured for the device in tris buffer were not particularly good when compared to the device measured in DI water in section 6.2.2. The device measured in this section, however, only achieved an on/off ratio of 800 when dry. This is at the bottom of the range for the nanowire devices and puts these results into some relative context.

The large leakage currents may also be due to this device being at the lower end of the device quality spectrum. It should be considered though, that tris buffer is substantially more conductive (by a factor of a few thousand [141]) than DI water and hence this may also be a factor contributing to the large leakage currents.

In summary, $\mathrm{ZnO}$ nanowire devices can be used as FETs in tris buffer and therefore should be viable in physiological conditions and other biological fluids. 


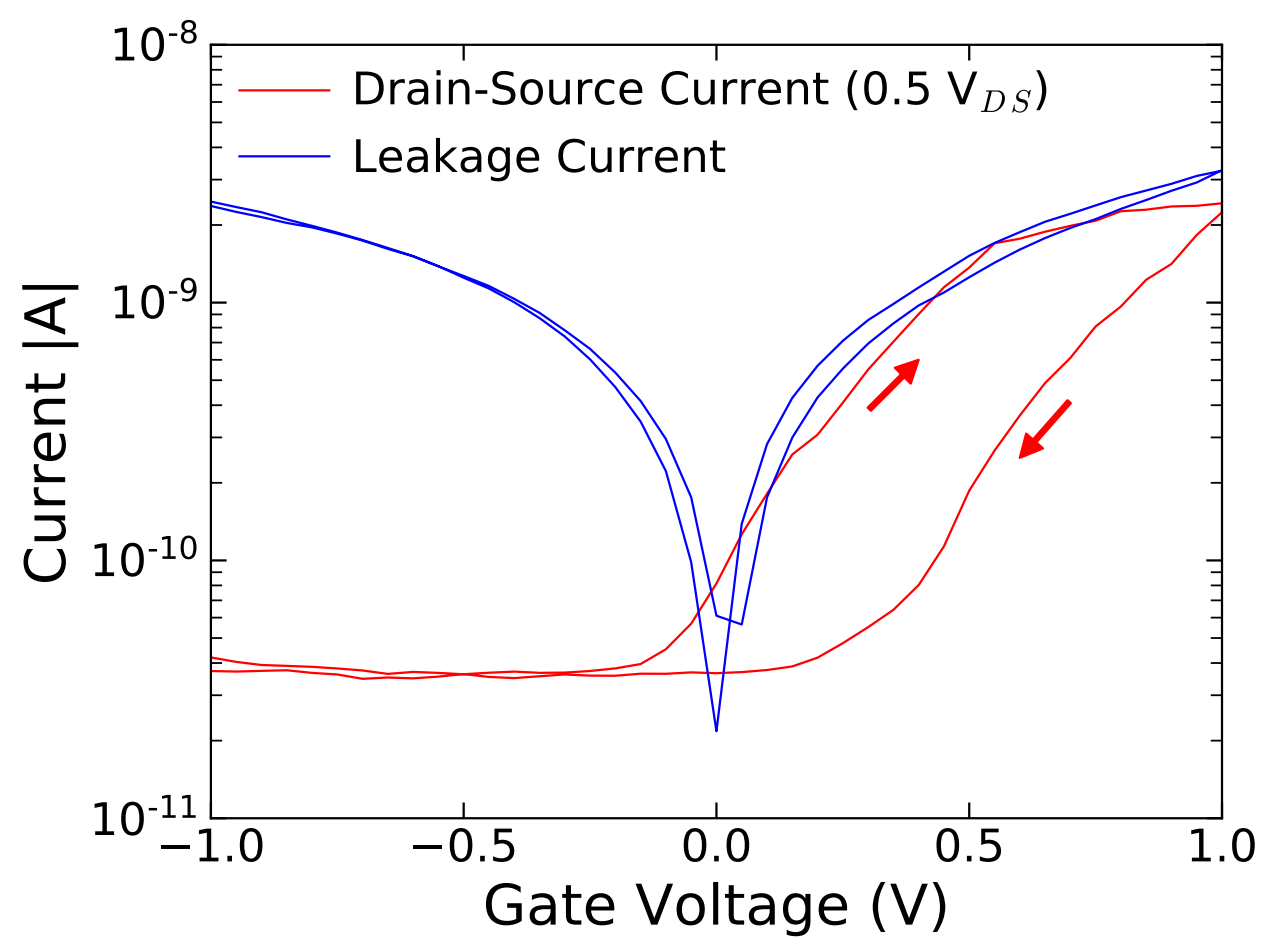

Figure 6.8: Transfer curve of a nanowire device backgated in tris buffer. This device has particularly large leakage currents, but still has an on/off ratio of 75 .

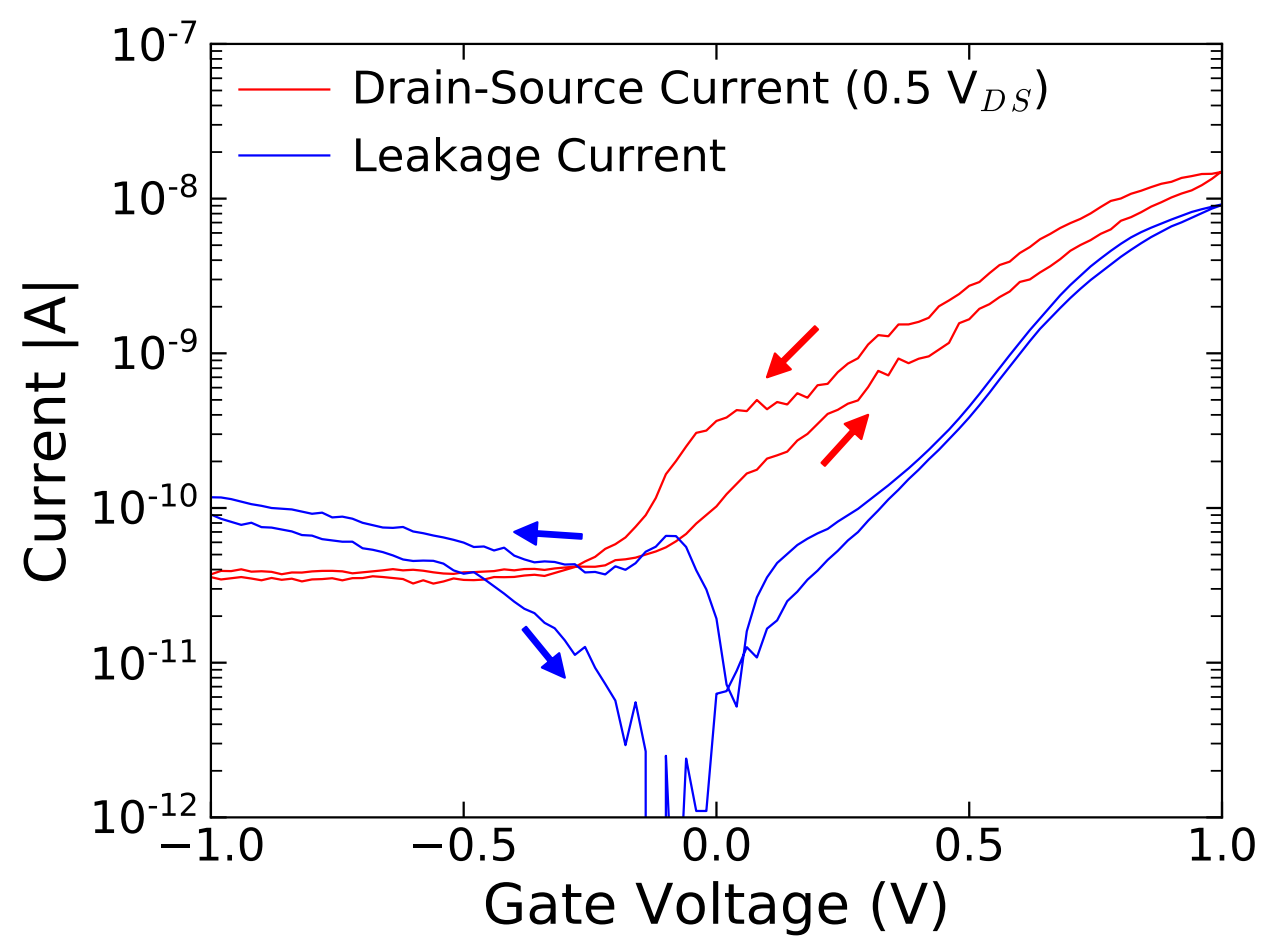

Figure 6.9: Transfer curve of a nanowire device topgated in tris buffer using a $\mathrm{Ag} / \mathrm{AgCl}$ electrode. 


\subsubsection{Liquid Gated (Ethanol)}

As described in section 3.2.2 surface oxygen species of $\mathrm{ZnO}$ can react with ethanol and free electrons back into the $\mathrm{ZnO}$, increasing the conductivity. This is the fundamental interaction that is used in the operation of $\mathrm{ZnO}$ ethanol gas sensors. The effect that this has on FETs that are fully immersed in liquid ethanol is not known as most liquid gated FETs use ionic liquids as the gate dielectric [54]. It is possible that reactions between the $\mathrm{ZnO}$ oxygen species and ethanol may saturate the nanowires with charge carriers and hence inhibit any field dependence.

To complete the characterisation of the nanowire samples, FET measurements were also made with the sample immersed in ethanol. Figure 6.10 shows the transfer curve of a $\mathrm{ZnO}$ nanowire device backgated through the $\mathrm{Si} / \mathrm{SiO}_{2}$ layer. The drain-source voltage is held at $0.5 \mathrm{~V}_{D S}$ and the gate voltage is swept from -1 to $+1 \mathrm{~V}_{G}$. An on/off ratio of $3 \times 10^{5}$ is achieved and the device has a forward threshold voltage of $0.24 \mathrm{~V}$ and backward threshold voltage of $0.51 \mathrm{~V}$. The minimum subthreshold swing is $140 \mathrm{mV}$ /decade and the maximum transconductance is $140 \mathrm{nS}$.

The same device is then topgated with a $\mathrm{Ag} / \mathrm{AgCl}$ electrode using the same sweep parameters. The transfer curve is shown in figure 6.11. In this configuration the device has similar characteristics with a minimum subthreshold swing of $110 \mathrm{mV} /$ decade and a maximum transconductance of $450 \mathrm{nS}$. The off current

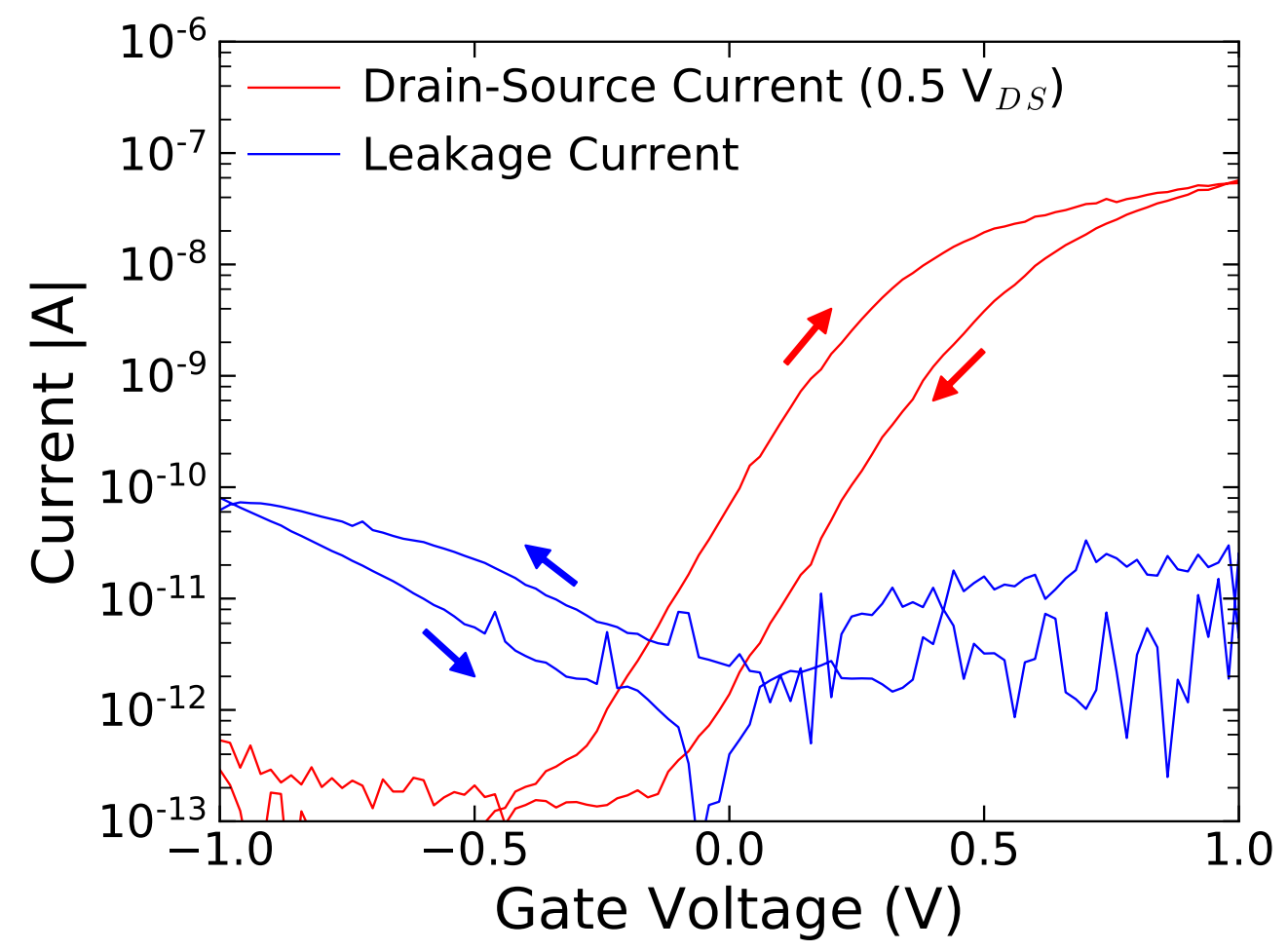

Figure 6.10: Transfer curve of a backgated nanowire device in ethanol. The on off ratio is $3 \times 10^{5}$. 
is somewhat larger than the backgated setup and hence the on/off ratio is smaller at $4 \times 10^{4}$. Some hysteresis is apparent and this device has a forward threshold voltage of $0.28 \mathrm{~V}$ and a backward threshold voltage of $0.15 \mathrm{~V}$.

This measurement shows that ethanol can be used as a liquid dielectric for $\mathrm{ZnO}$ nanowires. The ethanol has not saturated the $\mathrm{ZnO}$ nanowires with charge carriers and acts as an efficient liquid dielectric with good on/off ratios achieved in the narrow -1 to $1 \mathrm{~V}_{G}$ range.

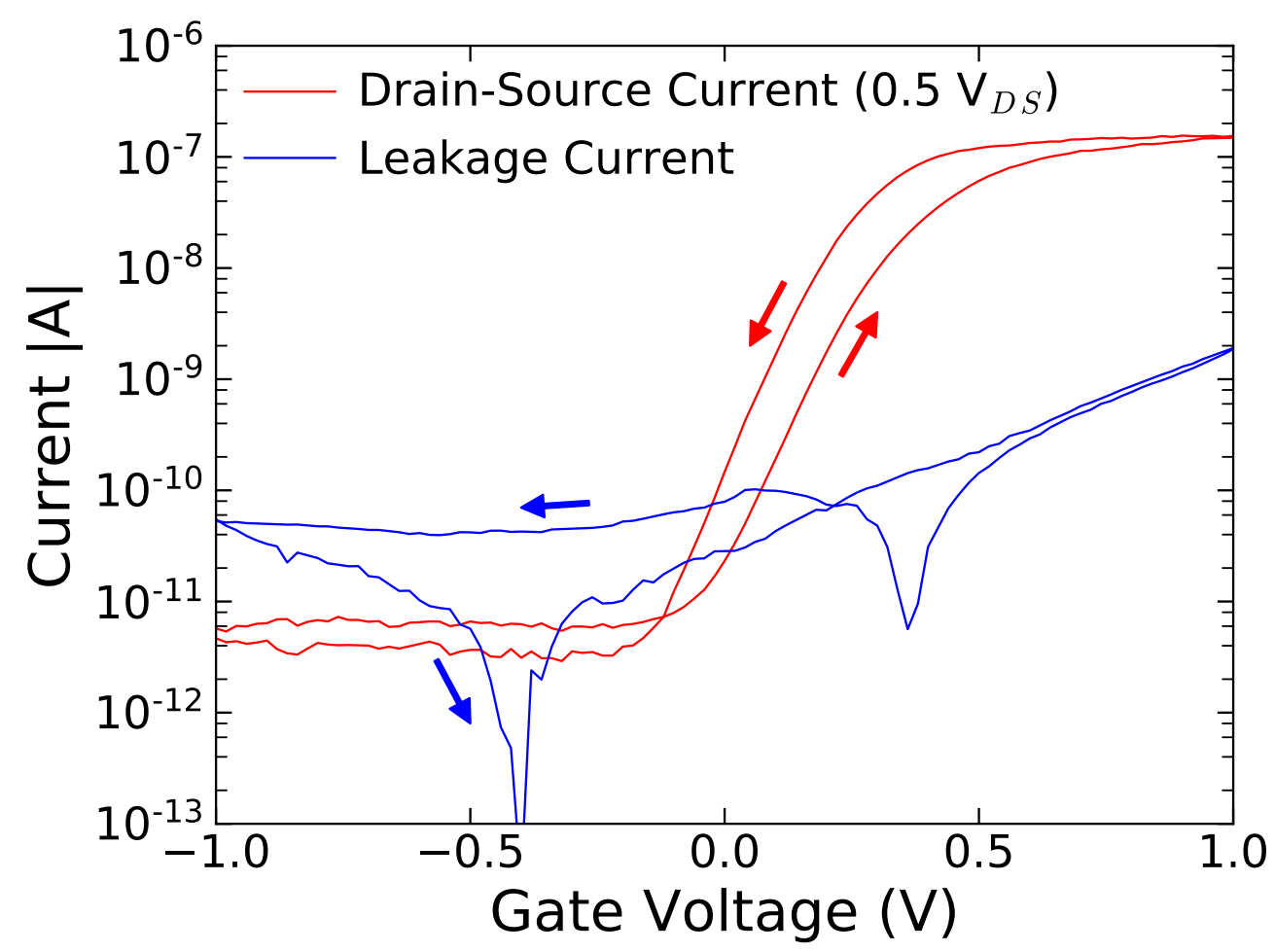

Figure 6.11: Transfer curve of a nanowire device topgated in ethanol using a $\mathrm{Ag} / \mathrm{AgCl}$ electrode. There is minimal hysteresis compared to the water gated devices.

\subsection{Hysteresis in ZnO Nanorods and Nanowires}

Under liquid conditions, both the nanorod and nanowire devices showed hysteresis between the forwards and backwards sweeps of their respective transfer curves. This is usually attributed to the effect of charge trapping groups, such as adsorbed water molecules, which become polarised and screen charge in the $\mathrm{ZnO}[106,108,111]$ (see section 3.2.4). In addition to this, however, both the nanorods and nanowires show hysteresis across repeated transfer sweeps.

A series of identical transfer curve measurements were taken for one of the nanorod devices in DI water, with a drain-source voltage of $0.5 \mathrm{~V}_{D S}$ and the gate 
voltage sweeping from -1 to $1 \mathrm{~V}_{G}$ and back. Likewise, a series of identical transfer sweeps were taken for a nanowire device with the same $\mathrm{V}_{D S}$ and $\mathrm{V}_{G}$ parameters. A representative set of transfer sweeps is shown for the nanorod device in figure 6.12 and for the nanowire device in figure 6.13.

There is clearly a change in both the nanorod and nanowire devices' transfer characteristics as the sweep number increases. The threshold voltage moves to a more positive gate voltage and there is a decrease in the maximum current at $1 \mathrm{~V}_{G}$. This shows that as the sample is tested there is a long term change being made to the device. Yuan et al. [142] discuss the interactions at the surface of $\mathrm{ZnO}$ with $\mathrm{H}^{+}$ and $\mathrm{OH}^{-}$groups. They identify that the $\mathrm{ZnO}-\mathrm{O}-\mathrm{H}$ bond is thermodynamically stable (bonding energy of $4.77 \mathrm{eV}$ ), but that a negative gate voltage can be used to desorb the hydroxyl groups. The desorption process would result in a decrease in carrier concentration and positive shift in threshold voltage. The trend seen between sweeps in figures 6.12 and 6.13 is also a positive shift in threshold voltage. Furthermore the effect is larger in the nanowires, which have a larger surface to volume ratio, as is expected for a surface interaction.

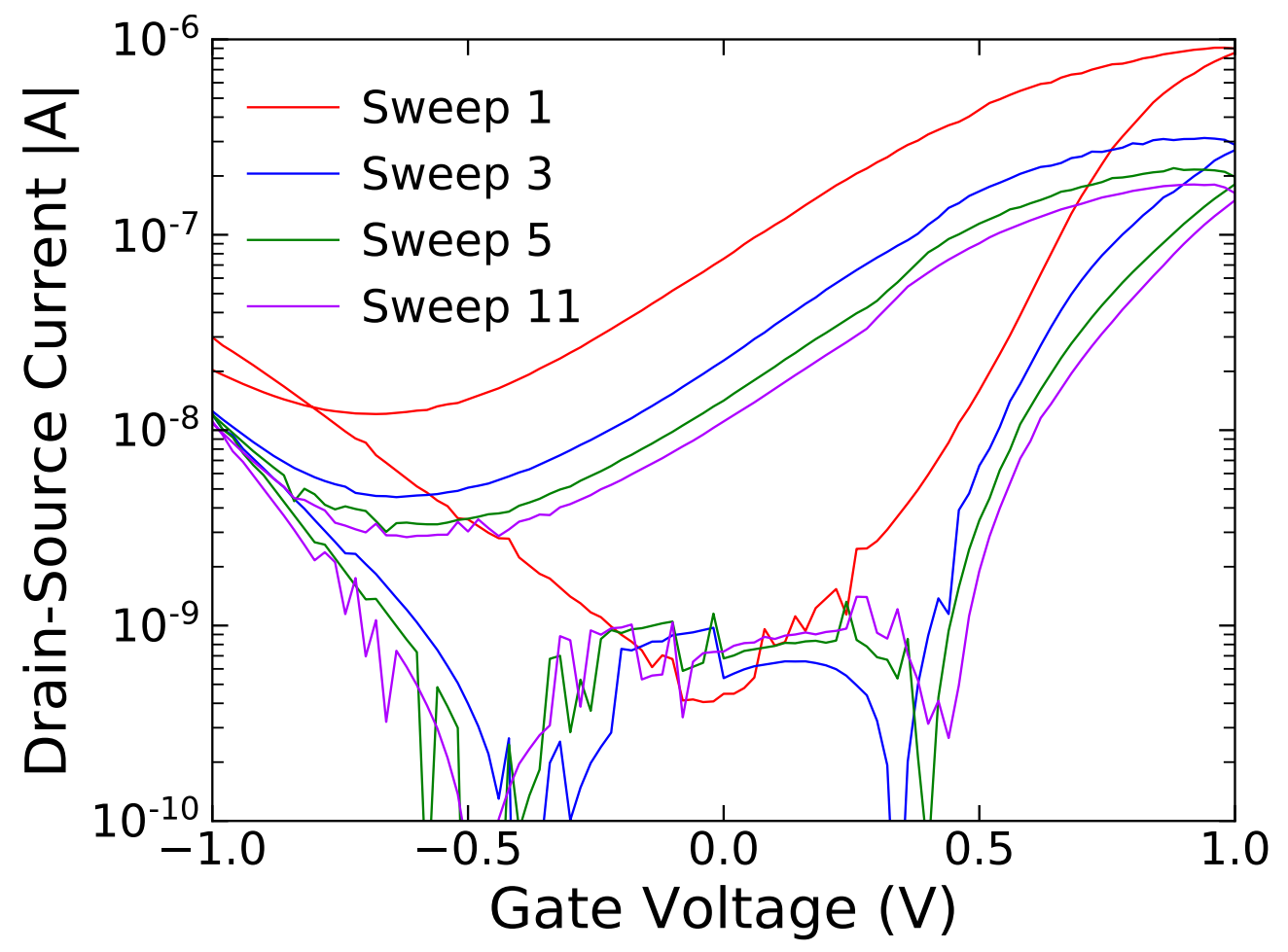

Figure 6.12: A sequence of identical transfer sweeps for a nanorod device in DI water were taken. Some representative examples are shown here with the sweep number as labelled. The threshold voltage shifts to a more positive value and the maximum current decreases. 
It is therefore likely that the surface of the $\mathrm{ZnO}$ is heavily hydroxylated. At the negative gate voltages the hydroxyl groups are removed at a certain rate, but due to the high concentration of hydroxyl groups on the $\mathrm{ZnO}$ surface the rate of re-hydroxylation at positive gate voltages is slower. Over numerous sweeps the $\mathrm{ZnO}$ surface will come to a new equilibrium with a lower hydroxyl density. This accounts for the lower rate of change in latter sweeps and shows that the surface chemistry of the $\mathrm{ZnO}$ nanorods and nanowires are coming to a new equilibrium. For more information on the interaction between water and $\mathrm{ZnO}$ see [142-144].

This hysteresis might cause issues when the devices are applied as a biosensor. Ideally a sensor should be able to reproduce the same result over many tests and hysteresis could make this impossible. The biofunctionalisation reactions, however, will chemically modify the surface of the $\mathrm{ZnO}$. This will likely change the hysteric behaviour, although it is yet to be determined to what extent this will be the case and whether it will impact on sensing measurements.

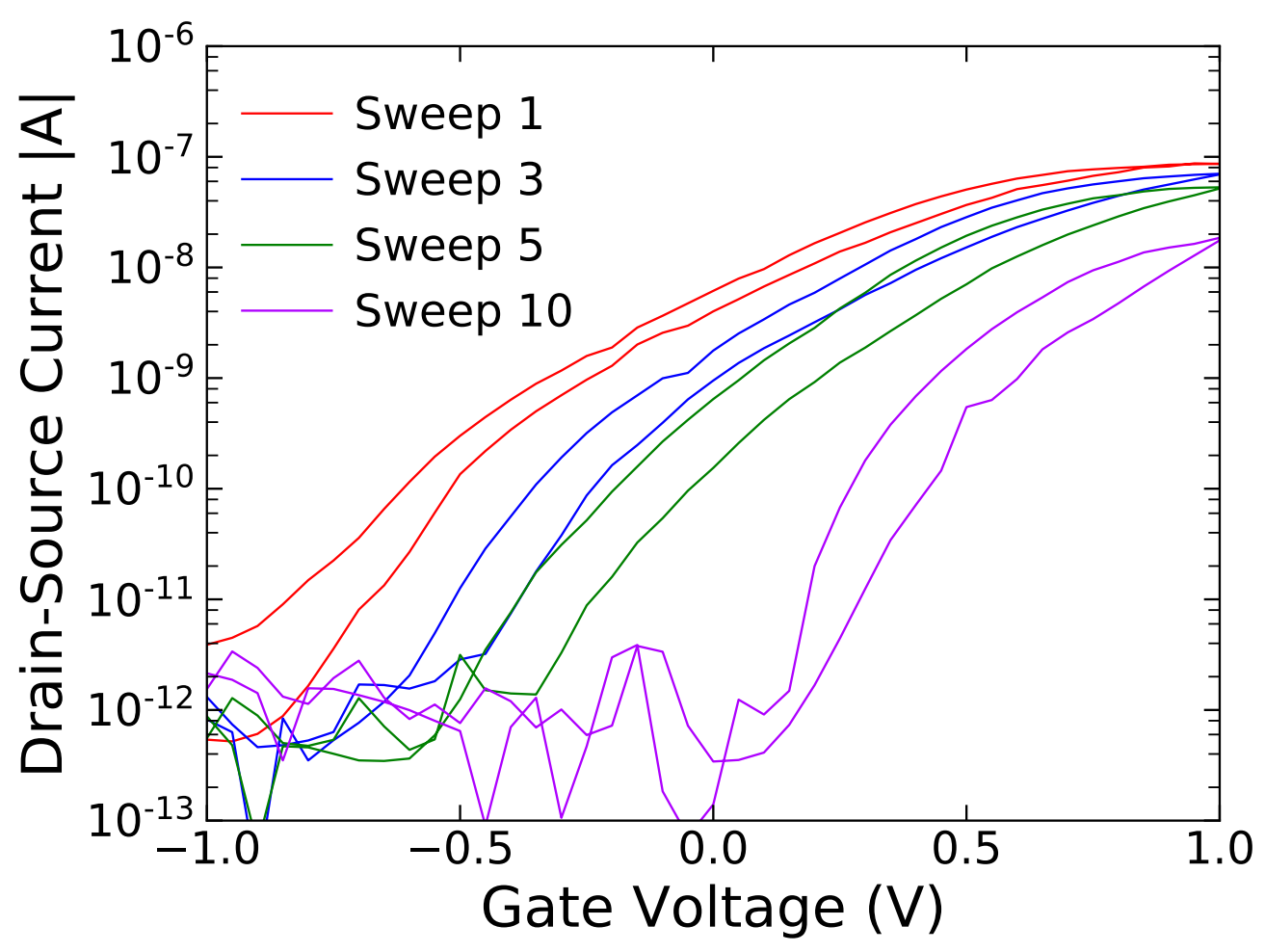

Figure 6.13: A sequence of identical transfer sweeps for a nanowire device in DI water were taken. Some representative examples are shown here with the sweep number as labelled. The changes between sweeps are larger than in the nanrord devices, attributed to the larger surface to volume ratio of the nanowires. 


\subsection{Conclusions}

Nanorod and nanowire devices were electrically characterised as FETs. The devices were tested by taking transfer curves in three different configurations. The devices were first measured dry using the $\mathrm{Si} / \mathrm{SiO}_{2}$ substrate as a backgate. Second, a PDMS well was placed on top of the device and filled with a given liquid. The measurements were repeated using the backgate and then lastly a $\mathrm{Ag} / \mathrm{AgCl}$ electrode was inserted into the liquid and used in a topgate configuration.

The nanorod devices showed negligible field dependence when dry. The nanowires, however, show strong field dependence, with typical on/off ratios of $10^{4}-10^{5}$.

Once immersed in DI water, the nanorods do show field dependence, with on/ off ratios of around $10^{3}$. The wet nanowires, however, have better FET characteristics, with on/off ratios at least an order of magnitude larger than the nanorods, and are therefore the preferred device platform as a sensor. Furthermore, the nanorods become highly conductive and lose field dependence when baked at high temperatures. The GOPS functionalisation procedure for biofunctionalisation requires that the SU-8 encapsulation layer be hardbaked and hence the nanorods can not be functionalised using this method. Hardbaking does not seem to affect the nanowires and chapter 7 will focus on attempts to functionalise the nanowire devices. 


\section{Chapter 7}

\section{Functionalisation Results}

This chapter follows the process of functionalising the $\mathrm{ZnO}$ nanowire devices. Electrical measurements are made after each step to track the effect of the chemical processes which are described fully in section 4.2. The nanorod devices were not functionalised as they could not be hardbaked without becoming highly conductive (see section 6.1.3). Hardbaking is required for the SU-8 to resist the toluene that the GOPS is diluted in.

\subsection{Surface Modification}

The first steps in the functionalistaion of $\mathrm{ZnO}$ require the adhesion of a linker molecule. In this case (3-Glycidyloxypropyl)trimethoxysilane (GOPS) is used. This is a silane that includes a long organic chain terminating in a epoxy group. The silane end can react and bind to the surface of metal oxides, while the epoxy end can react with amines. GOPS can therefore link $\mathrm{ZnO}$ to amine terminated aptamers.

\subsubsection{Oxygen Plasma}

The initial fictionalisation step is to expose the samples to an oxygen plasma. This ensures that the surface of the wires have been fully oxygenated with reactive oxygen groups $\left(\mathrm{O}^{2-}, \mathrm{O}^{-}\right.$and $\left.\mathrm{OH}^{-}[124,129,130]\right)$ in preparation for the silanisation reaction with the linker molecule, GOPS.

The samples are exposed to oxygen plasma for 30 minutes. A transfer curve of the device measured in section 6.2.1 after exposure to oxygen plasma is given in figure 7.1. The sample is kept dry and gated through the $\mathrm{Si} / \mathrm{SiO}_{2}$ layer. $\mathrm{A}$ comparison of the transfer curves for each procedure is given in figure 7.3. The on/off ratio of the device is effectively unchanged by the oxygen plasma at 2.2 $\times 10^{4}$, compared to $3.3 \times 10^{4}$ before the plasma exposure. The threshold voltage is only slightly different at $0.4 \mathrm{~V}$ (vs previously $-0.8 \mathrm{~V}$ ), however, the most notable change is the steepness of the transfer curve. The minimum subthreshold swing has decreased from $450 \mathrm{mV} /$ decade to $170 \mathrm{mV}$ / decade and the transconductance has increased from $40 \mathrm{nS}$ to $69 \mathrm{nS}$

The highly reactive oxygen plasma has the potential to fill any oxygen vacancies on the surface of the nanowires. This would decrease the overall conductivity of 
the nanowires and this has been observed in literature $[129,130]$. It is not clear whether this is the case with the device measured in Figure 7.1. Although the drain-source current at $\mathrm{V}_{G}=0$ is small (the device is effectively in the off state for both the forward and backward sweep), the maximum on current is nearly unchanged after the oxygen plasma exposure. This is in contrast to at least one report in literature [129] where the maximum on current decreases with plasma exposure time.

The increase in source-drain current at negative voltages is likely due to a larger leakage current which peaks at $10^{-9} \mathrm{~A}$, compared to previously peaking at $10^{-10}$ A (see figure 6.5).

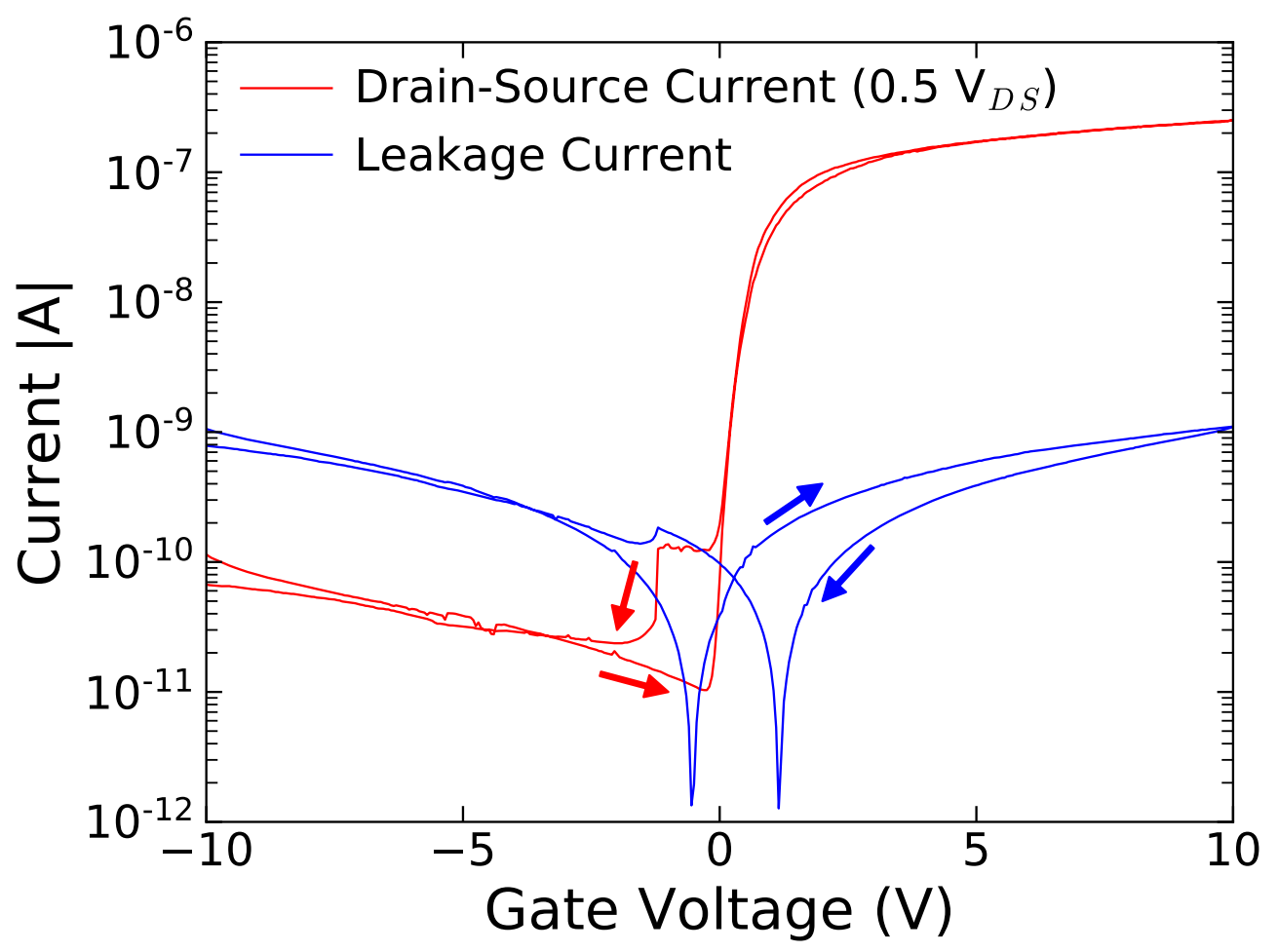

Figure 7.1: Transfer curve of a nanowire device after it has been exposed to an oxygen plasma for 30 minutes. The device shows a remarkably steep transfer curve.

\subsubsection{GOPS Functionalisation}

After the oxygen plasma exposure, the devices are immersed in a $10 \mathrm{mM}$ GOPS solution in water free toluene for six hours. The transfer curve of the sample after GOPS modification is given in figure 7.2.

There is a clear change in the transfer curve. A very large hysteresis is present with a forward sweep threshold voltage of $-3.9 \mathrm{~V}$ and a backward sweep threshold voltage of $4.6 \mathrm{~V}$. The large hysteresis is consistently attained across different 


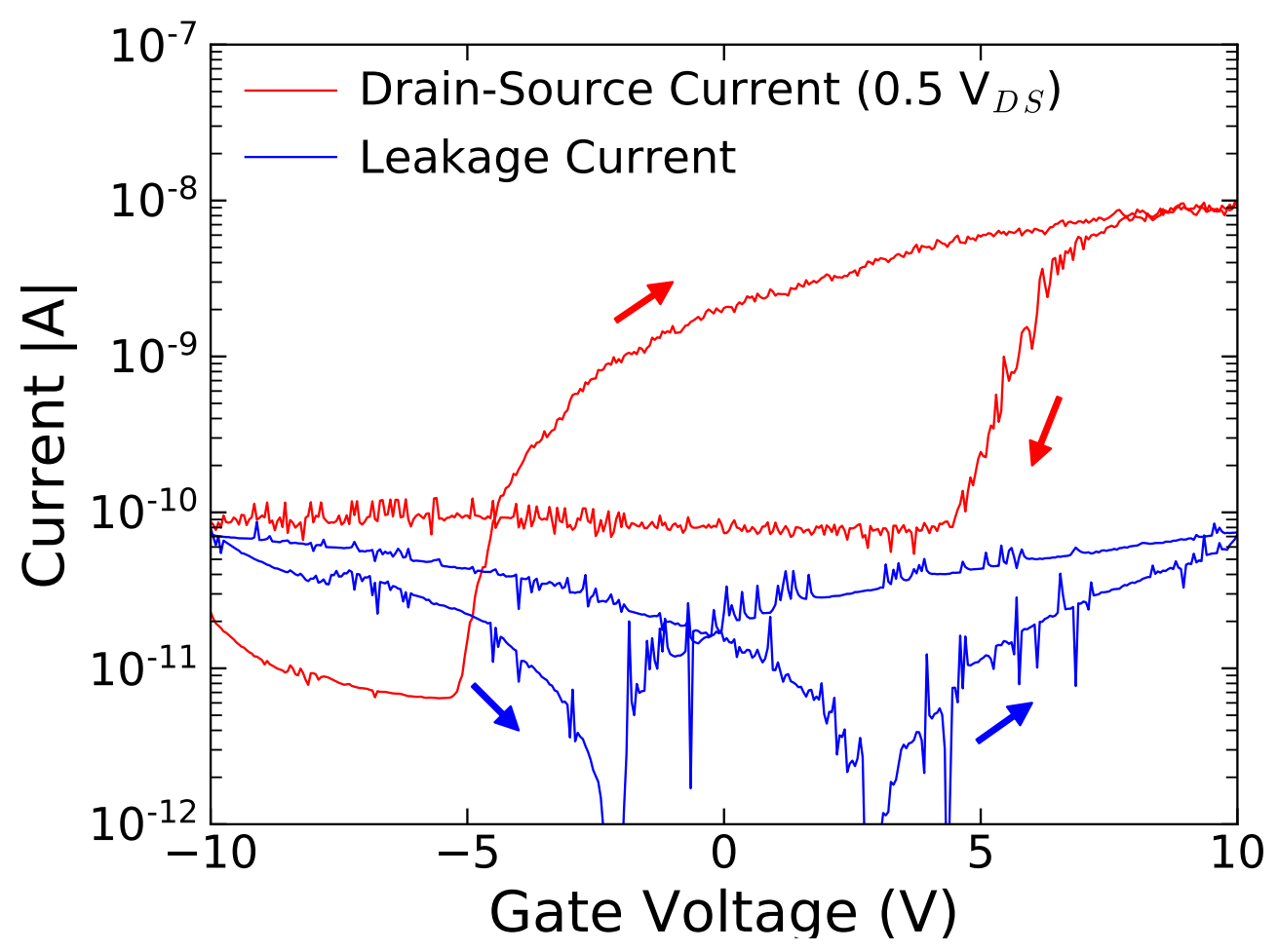

Figure 7.2: Transfer curve of a nanowire device after the GOPS modification. There is a clear change in behaviour, implying a modification of the surface states/chemistry.

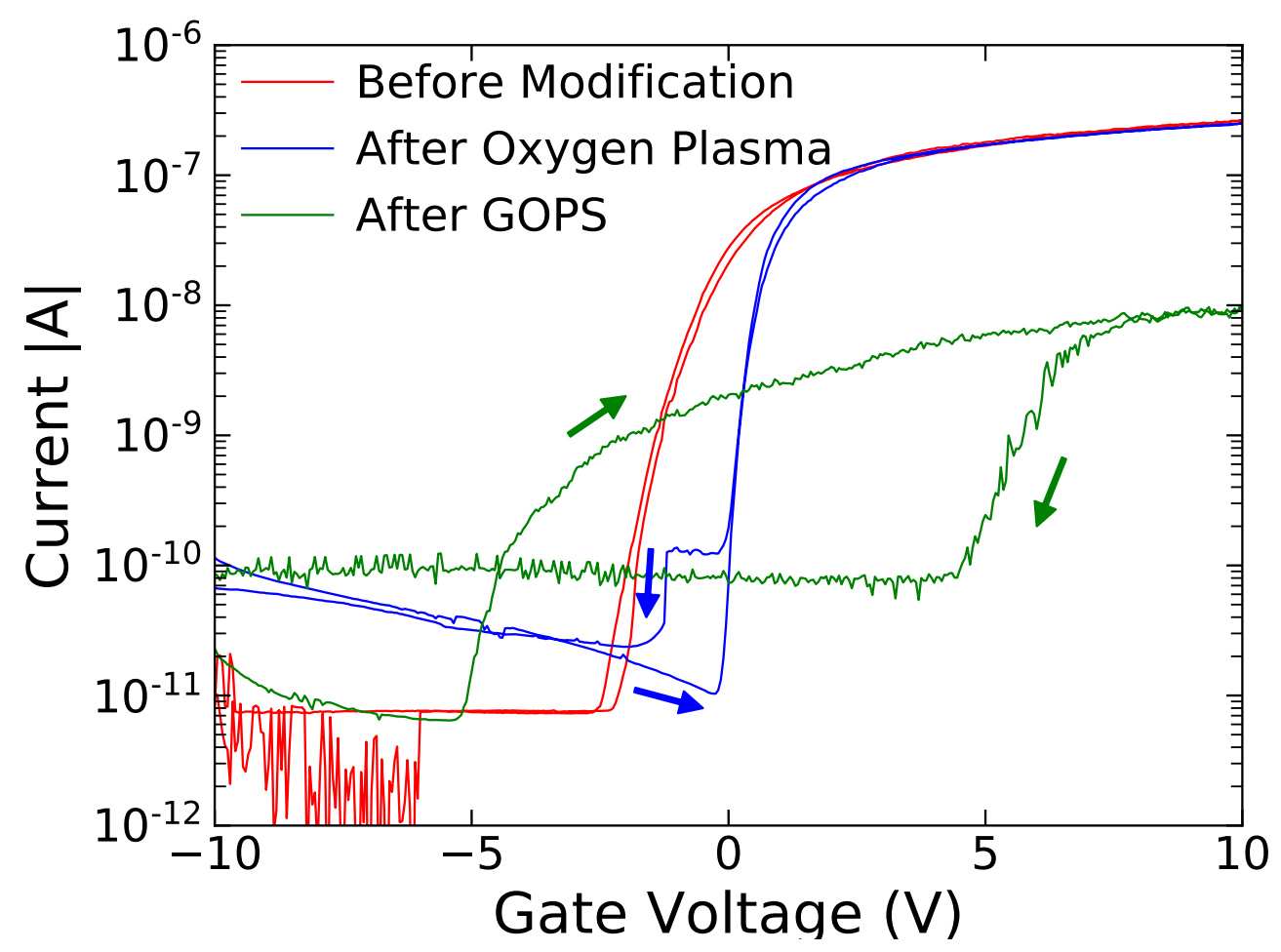

Figure 7.3: A comparison of the transfer sweeps of a single nanowire device after each of the functionalisation steps 
devices. The GOPS modified nanowires also switch much slower, with a minimum subthreshold swing of $790 \mathrm{mV} /$ decade and a transconductance of $7.9 \mathrm{nS}$.

These values are substantially different to the unmodified wires and this is most obvious in figure 7.3. As described in section 3.2.4 the hysteresis in ZnO FETs is typically linked to the polarisation of charge trapping groups on the $\mathrm{ZnO}$ surface. The clear change in electric behaviour of the nanowires implies that the surface states of the $\mathrm{ZnO}$ nanowires have changed and this suggests that the GOPS has successfully reacted with the $\mathrm{ZnO}$ surface.

\subsection{Aptamer Functionalisation}

The next step is to react the GOPS modified nanowires with amine terminated aptamers. For this step the aptamers were diluted in $2 \mathrm{mM}$ tris buffer, pipetted onto a sample and left for two hours.

After the reaction with the aptamers, the nanowires did not conduct and there was no measurable field dependence. On closer inspection with an optical microscope it appeared that the nanowires had disappeared (this was confirmed using an SEM). The functionalisation method used in this thesis was successfully used in $[19,124]$, although the authors dilute the aptamers in phosphate-buffered saline (PBS) rather than tris buffer. PBS is very similar to tris buffer and replicates physiological conditions. Functionalisation with PBS was also trialled, but also led to the dissolution of the nanowires.

The dissolution of the nanowires in tris buffer is surprising given that the nanowire devices were not dissolved during the measurements in section 6.2.3. The time period over which the nanowires dissolved was therefore investigated. A PDMS well was placed over a GOPS modified device and had $0.5 \mathrm{~V}_{D S}$ applied. The gate voltage was held at $1 \mathrm{~V}_{G}$ (through the backgate) to ensure that the sample is always in the on state and a non-zero current can be measured. After one minute the PDMS well is filled with $2 \mathrm{mM}$ tris buffer.

Upon application there is a spike in the drain-source current. At $1 \mathrm{~V}_{G}$ the device should be in the on state in tris buffer, but only intermediate (ie. neither fully on or off) when dry. Therefore a rapid increase in current is to be expected. The physical application of the tris buffer, however, can cause a lot of electrical noise and the data values at this point may be unreliable. None the less, it can be observed that the current rapidly decays. This can be very loosely fitted with an exponential decay, giving a decay constant of $-0.0195 \mathrm{~s}^{-1}$ and a current half-life of proximately 36 seconds. 
In section 6.2.3 measurements of nanowire devices in tris buffer were made. Each transfer curve measurement takes a few minutes to complete and therefore would not be possible if the wires decayed in a matter of seconds as in figure 7.4. To investigate this, another decay curve was measured with an unfunctionalised nanowire device. The device was driven at $0.5 \mathrm{~V}_{D S}$ and held at $1 \mathrm{~V}_{G}$ to ensure that the device is in the on state. Tris buffer was pipetted into the PDMS well on top of the device after one minute. The result is shown in figure 7.5.

The unmodified wires do not rapidly decay like those which have been functionalised. This means that there must be some change in the chemical properties of the nanowires during the functionalisation process that makes the nanowires open to attack by the tris buffer. The removal of some unidentified coating (eg. residue photoresist) on the nanowires is unlikely as SEM micrographs (such as in figure 5.11) do not show any evidence of this.

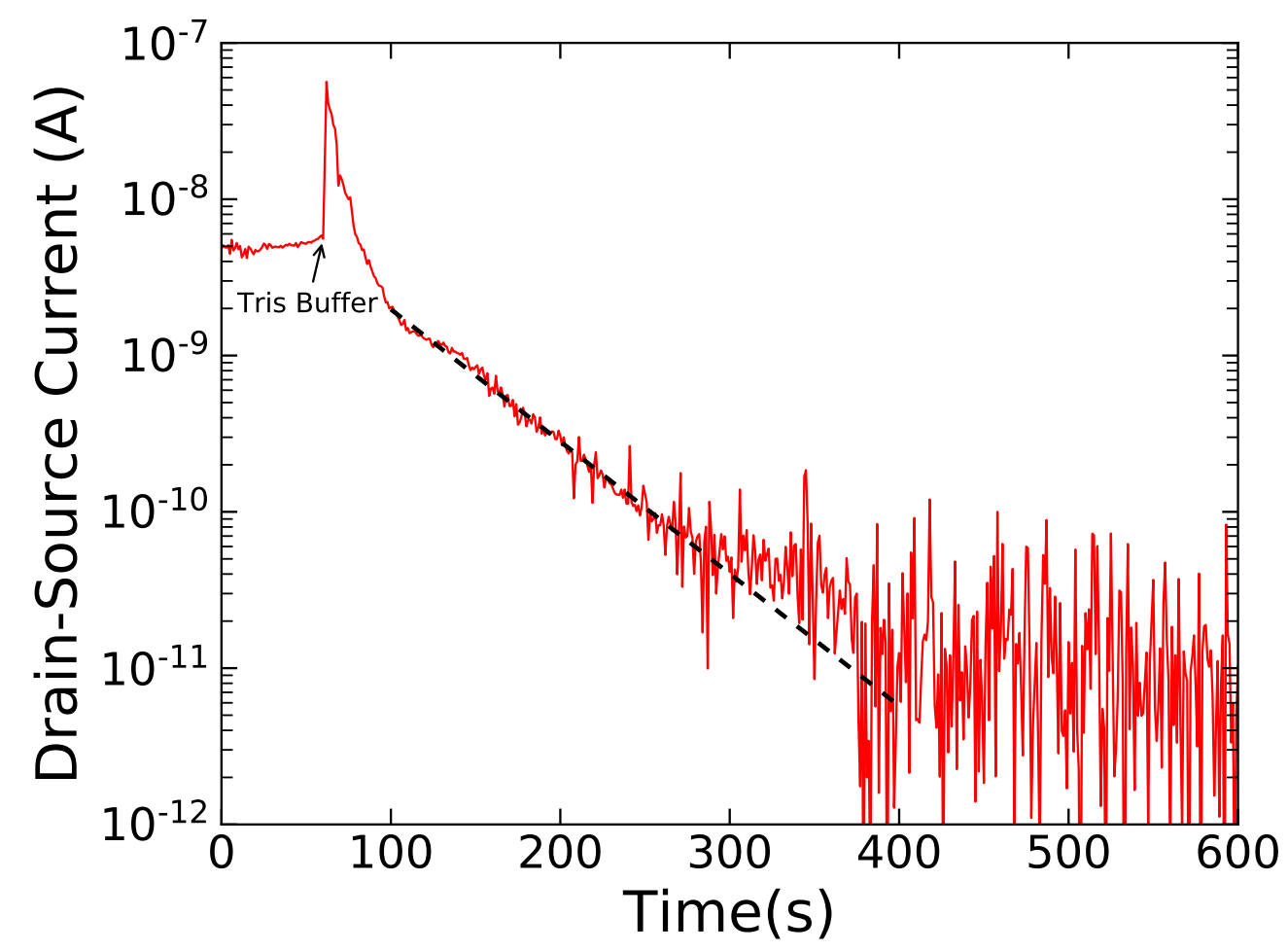

Figure 7.4: A GOPS modified sample is driven at $0.5 \mathrm{~V}_{D S}$ and held at $1 \mathrm{~V}_{G}$ through the backgate. Tris buffer is applied at 60 seconds and the current rapidly decays (after an initial spike). The decay can be loosely fitted with an exponential decay (black line). 


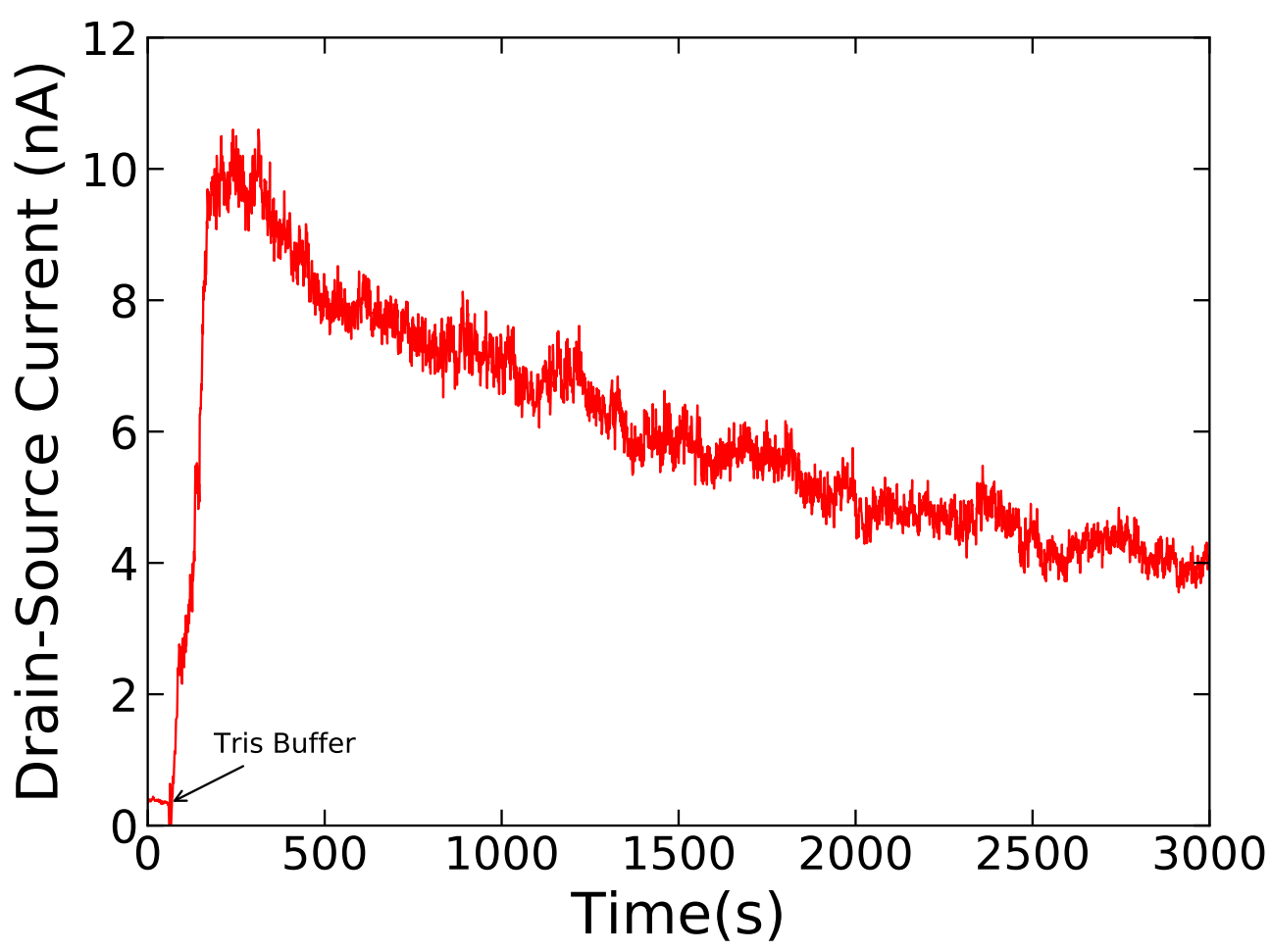

Figure 7.5: A nanowire sample that has not been modified with GOPS does not rapidly degrade like in figure 7.4. The sample is driven with 0.5 $\mathrm{V}_{D S}$ and held at $1 \mathrm{~V}_{G}$.

\subsection{Discussion}

The degradation of the $\mathrm{ZnO}$ nanowires in tris buffer after GOPS modification is surprising. The method used in this thesis is taken from $[19,124]$. In both [19] and [124] the $\mathrm{ZnO}$ nanowires are fabricated using a vapour liquid solid method and have diameters of about $100 \mathrm{~nm}$. The functionalisation with aptamers in buffer is successful and there is no discussion of degradation. Furthermore there are other examples of successful aptamer functionalisation, that involve the use of $\mathrm{ZnO}$ nanowires in conjunction with buffer solutions [16, 19, 124-126, 131,139], but this is without any discussion on the stability of the nanowires.

There has, however, been some limited study into the issue of the chemical stability of $\mathrm{ZnO}$ nanostructures [145-147]. Zhou et al. [145] found that large nanowires of approximately $1.5 \mu \mathrm{m}$ in diameter, are etched in horse blood serum and reduce in diameter to about $500 \mathrm{~nm}$ after six hours. These same nanorods also showed similar degradation after only 30 minutes in DI water. Müller et al. [146] investigated nanowires (diameter approximately $120 \mathrm{~nm}$ ) in lysosomal fluid and observed that the nanowires showed jagged surfaces after only 10 seconds of exposure and were completely dissolved after two minutes. 
The most in depth investigation into the chemical stability of $\mathrm{ZnO}$ nanowires in biological fluids seems to be by Cheng et al. [147]. They investigate the etching mechanism of $\mathrm{ZnO}$ nanowires in simulated physiological solution (SBS). The etching process is identified to be linked to the formation of reactive $\mathrm{ZnO}(\mathrm{OH})_{4}{ }^{2-}$ complexes on the $\mathrm{ZnO}$ surface. These form at defect sites such as ionised oxygen vacancies, antisite oxygen and zinc interstitials. Furthermore they test nanowires fabricated from both a vapour-solid method and a hydrothermal method. The concentration of defects was higher in the hydrothermally grown nanowires and as a result degraded faster in SBS than the nanowires fabricated with the vapoursolid method.

The stability of $\mathrm{ZnO}$ nanowires in biological liquids is therefore highly dependant on the degree of crystalinity and dopant level. The unfunctionalised nanowires in this thesis were relatively stable in tris buffer and this would imply that the functionalisation process is causing a chemical or structural change to the $\mathrm{ZnO}$ surface. Whether this is due to the linking of GOPS or perhaps an increase of defects due to the oxygen plasma is unclear. Further work would be necessary to identify which stage is making the nanowires chemically unstable.

\subsection{Conclusion}

Nanowire devices were chemical modified with GOPS in order to be functionalised with amine terminate aptamers. Transfer sweeps of the nanowire devices between steps suggested that the adhesion of GOPS was successful. This was concluded from the large hysteresis in figure 7.2, implying a substantial change in the surface states on the nanowires. Upon functionalisation with aptamers in tris buffer, however, the nanowires dissolved. The rate of decay was measured as a function of the drain-source current and was found to be loosely exponential, with a decay constant of $-0.0195 \mathrm{~s}^{-1}$. Unfunctionalised $\mathrm{ZnO}$ nanowires, however, were relatively stable in tris buffer. There has been limited study on the stability of $\mathrm{ZnO}$ nanostructures in biological fluids, but the dissolution may be linked to the concentration of defects in the $\mathrm{ZnO}$. Further study would be necessary to identify the exact process that leads to the nanowire degradation seen in this thesis. 


\section{Chapter 8}

\section{Gas Sensing}

In this brief chapter nanorod and nanowire devices are tested as sensors for humidity (water vapour) and ethanol vapour. This uses the gas rig setup described in section 4.4. These measurements involve sealing the device in an airtight chamber and measuring the change in drain-source current as different concentrations of solvent vapour flow through the chamber. As described in section 3.2.2, the operation of $\mathrm{ZnO}$ gas sensors rely on the chemical interaction between $\mathrm{ZnO}$ and vapour molecules. The gate is, therefore, not used and these devices act as chemiresistors (i.e. a resistor that changes its electrical resistance due to chemical interactions with the environment). The results show that nanowire devices might be viable as gas sensors, but further development of the setup is necessary to achieve more quantitative results.

\subsection{Humidity Sensing}

There are a number of examples in the literature that use $\mathrm{ZnO}$ nanorods [8587] or nanowires for the sensing of humidity $[84,85,88]$. Both the nanorod and the nanowire devices used in this thesis might therefore be suitable as humidity sensors. Although the nanorod devices showed no field dependence when dry, if compared with the drain-source current of a nanorod device at $0 \mathrm{~V}_{G}$ when dry (figure 6.1), to when it is wet (figure 6.2), the current differs by about an order of magnitude. Gas sensing allows the device to reach a sort of intermediate between a dry and wet environment. It may, therefore, be possible to see a change in the drain-source current as a function of vapour concentration. Also, Fan et al. $[74,98]$ showed that sensitivity of $\mathrm{ZnO}$ nanowires to $\mathrm{O}_{2}$ increases as their radius decreases. This means that the much thinner nanowires might show a better signal than the nanorods.

\subsubsection{Nanorods}

The response of a nanorod device to humidity was measured and is shown in figure 8.1. The device is driven with a constant $0.1 \mathrm{~V}_{D S}$ so that $\mathrm{I}_{D S}$ is approximately a few $\mathrm{nA}$ and easily measured by the parameter analyser. The dilution line is set at a constant flow of $100 \mathrm{sccm}$ of $\mathrm{N}_{2}$ and the sample is initially left to settle for six minutes. The vapour line is then activated and $20 \mathrm{sccm}$ is pumped through a bubbler containing DI water. The vapour line is shut off after another six minutes. 
This process is repeated with a six minute wait and six minute vapour exposure for flows of 40, 60 and $80 \mathrm{sccm}$.

There are no clear spikes in the drain-source current when the device is exposed to humidity. Therefore the nanorods have no response to humidity under these conditions.

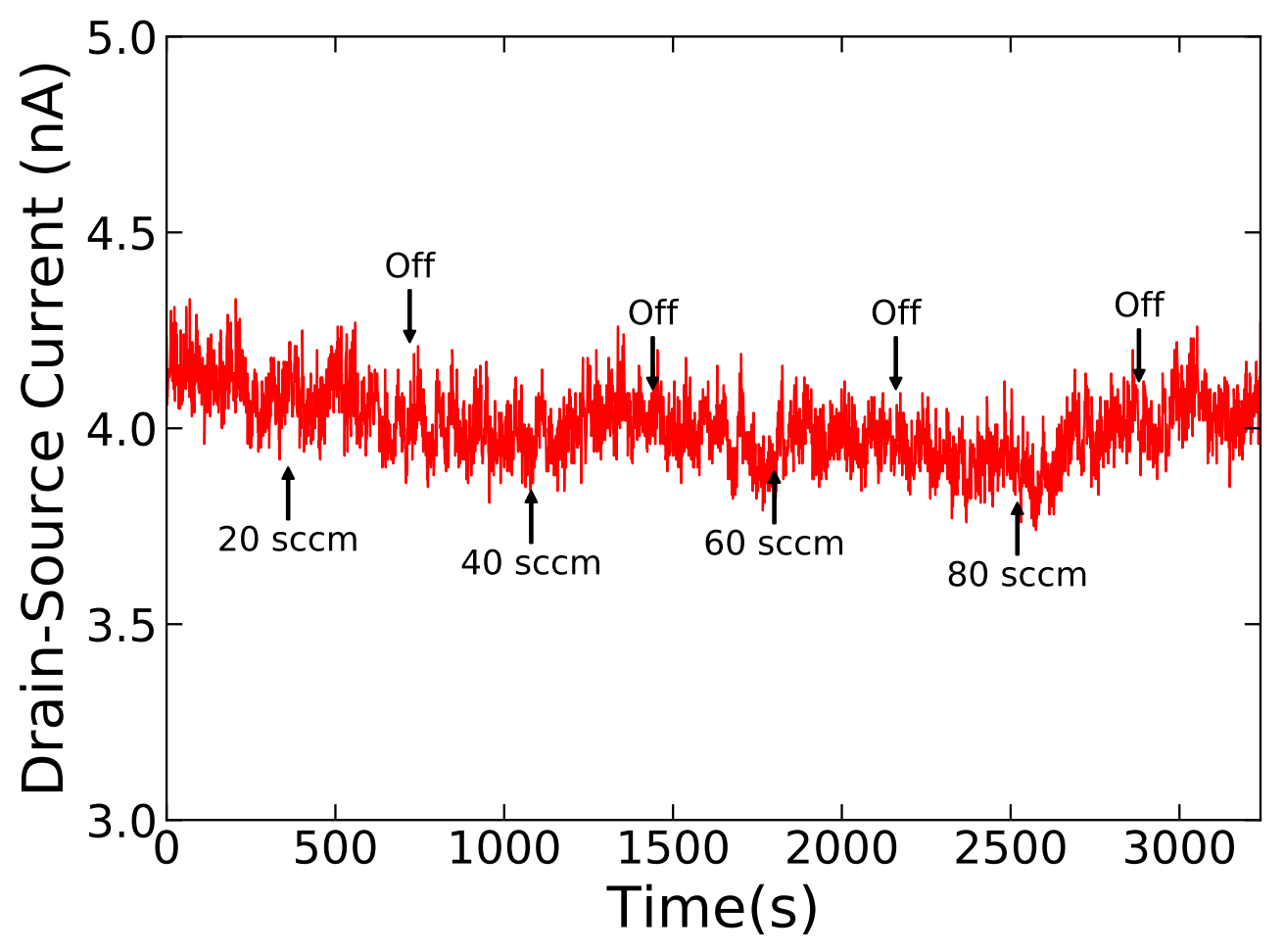

Figure 8.1: Humidity sensing with a nanorod device. A constant flow of $100 \mathrm{sccm}$ is pumped through the dilution line. The vapour line is set to the labelled flow rates. The device shows no response to humidity

\subsubsection{Nanowires}

Figure 8.2 shows a humidity sensing measurement for a nanowire device. The device is driven with a constant voltage of $0.5 \mathrm{~V}_{D S}$ and a constant flow of 100 $\mathrm{sccm}$ of $\mathrm{N}_{2}$ is driven through the dilution line. The device is first left to settle for five minutes before flowing $20 \mathrm{sccm}$ through the vapour line and a bubbler filled with DI water. The vapour line is shut off after one minute and the device left to settle for five minutes. This process is repeated with a flow of 40, 60, 80 and 100 $\mathrm{sccm}$ through the vapour line. Note that the SU-8 encapsulation layer ensures that only the nanowires are exposed to atmosphere as in figure 5.12 (any signal can then be attributed to the nanowires rather than the nanorods).

Exposure to humidity should increase the conductivity of the nanowires (water reduces $\mathrm{ZnO}$ [88]). There are very small increases in the data at the points where 
the vapour line is turned on, however, the device clearly does not have a strong response to humidity. Furthermore these increases could be attributed to noise, in particular the movement and vibrations caused by the mass flow controllers or the bubbler. The humidity sensing of this device is therefore either zero or negligible.

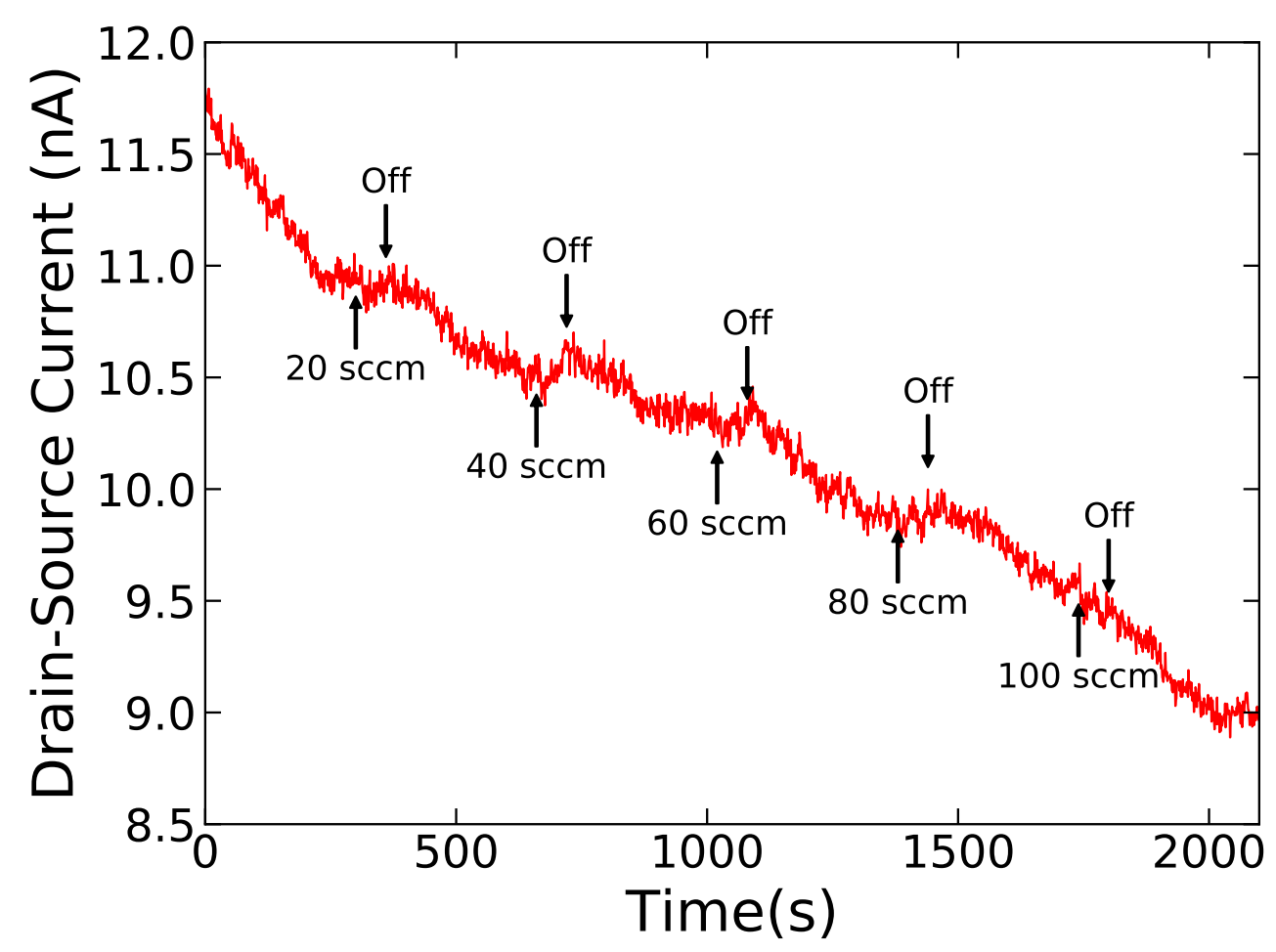

Figure 8.2: Humidity sensing with a nanowire device. A constant flow of $100 \mathrm{sccm}$ is pumped through the dilution line. The vapour line is set to the labelled flow rates. The small rises in the data (when vapour line is turned on) indicates that the device might be responding to the humidity. The response is extremely small and can not be confirmed to be as a result of humidity interaction.

\subsubsection{Discussion}

Humidity sensing with $\mathrm{ZnO}$ nanowires [84, 85] and nanorods [85-87] can typically be achieved at room temperature. Fang et al. [86] demonstrated that a dispersed thin film of $\mathrm{ZnO}$ nanorods had a reduction in resistance by a factor of $10^{5}$ as relative humidity was increased from $7 \%$ to $95 \%$. Both the nanorods and nanowires in this thesis show no or negligible response. The examples of $\mathrm{ZnO}$ humidity sensors in literature are fabricated from dispersed films of nanorods/nanowires, rather than orientated arrays of nanorods and nanowires like the devices in this thesis. The difference in device structure, however, should not be a key factor as humidity sensing with $\mathrm{ZnO}$ is attributed to the chemical reactions between $\mathrm{H}_{2} \mathrm{O}$ molecules and oxygen ion species on the surface of the 
$\mathrm{ZnO}$. The product of this reaction is the formation of $\mathrm{OH}$ groups on the $\mathrm{ZnO}$ surface and the release of electrons for conduction, increasing the conductivity of the $\mathrm{ZnO}$ [88]. As this is a surface reaction we would expect a difference in sensitivity between nanorods and nanowires (due to different surface to volume ratio), but the overall device structure should not be fundamentally important. The lack of response may suggest that the surface of the nanorods/nanowires are already saturated with $\mathrm{OH}$ groups, making humidity sensing impossible. This was also considered as a possible cause of the hysteresis effects observed in section 6.3. Alternatively the nanorods and nanowires may be covered in some other contaminant, such as photoresist residue, blocking any interaction. SEM images (e.g. figures 5.9 and 5.11), however, show that this is not the case.

\subsection{Ethanol Sensing}

Ethanol vapour sensing with $\mathrm{ZnO}$ nanowires $[89,90,92-96]$ and nanorods $[91,96$, 97] has also been conducted in the literature (Note that of these examples only [92] fabricate their nanowires using a hydrothermal method). The nanorod and nanowire devices will therfore be tested as ethanol sensing.

\subsubsection{Nanorods}

Another nanorod device was tested to see whether the nanorods would respond to an organic solvent, ethanol. Figure 8.3 shows the response. The sample is driven with a constant $0.1 \mathrm{~V}_{D S}$ and a constant $100 \mathrm{sccm}$ is pumped through the dilution line. The device is first left to stabilise for two minutes. The vapour line passes through a bubbler of ethanol and is activated for 10 minute periods for flow rates of 20,40,60 and $80 \mathrm{sccm}$. The vapour line is shut off for 10 minutes between each rate so that the sample can come back to equilibrium.

As with the humidity sensing, the nanorods show no response to ethanol vapour.

\subsubsection{Nanowires}

The same test used for humidity is repeated with ethanol. The device is driven with a constant $0.5 \mathrm{~V}_{D S}$ and the dilution line is set to a constant $100 \mathrm{sccm}$. The vapour line is turned on for one minute, with five minute rests in between the different flow rates of 20,40,60, 80, $100 \mathrm{sccm}$. The ethanol sensing measurement is given in figure 8.4 . 


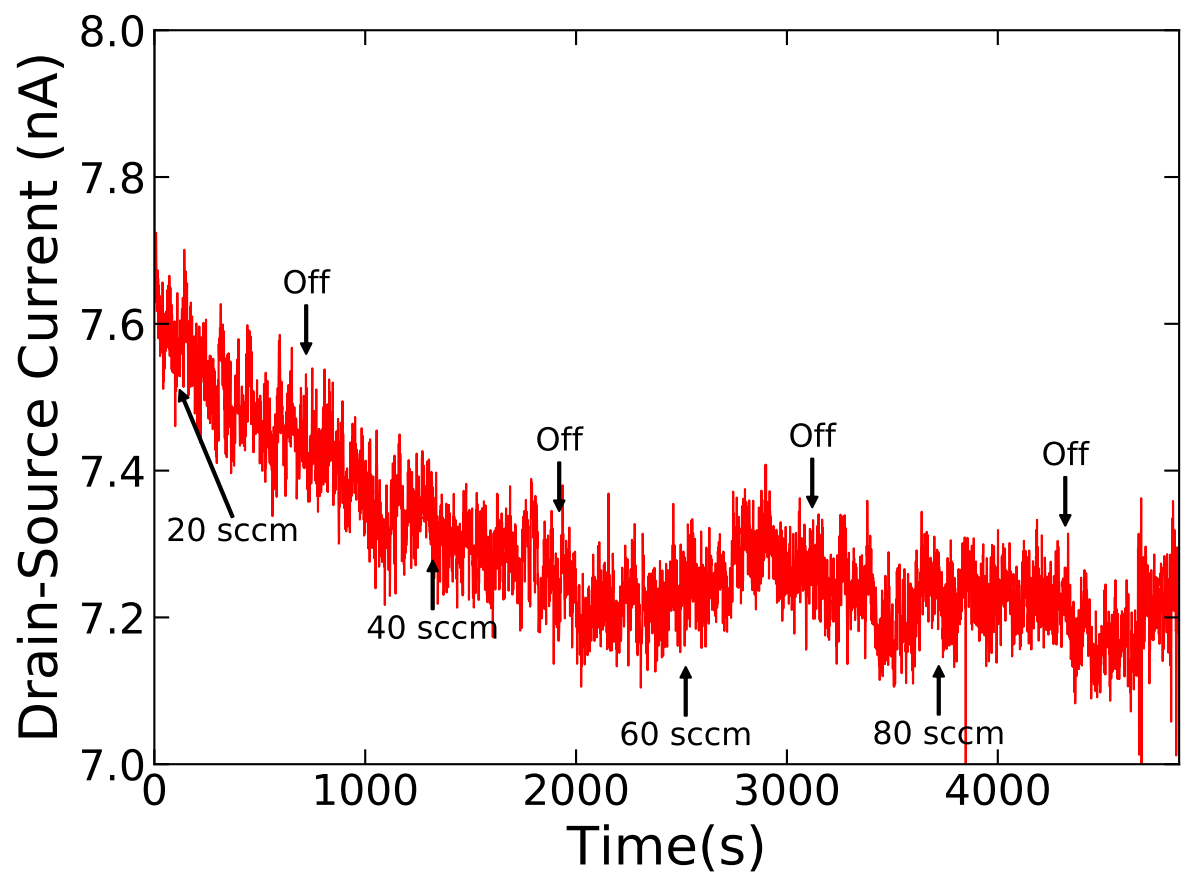

Figure 8.3: Ethanol sensing with a nanorod device. A constant flow of $100 \mathrm{sccm}$ is pumped through the dilution line. The vapour line is set at the labelled flow rates. The device shows no response

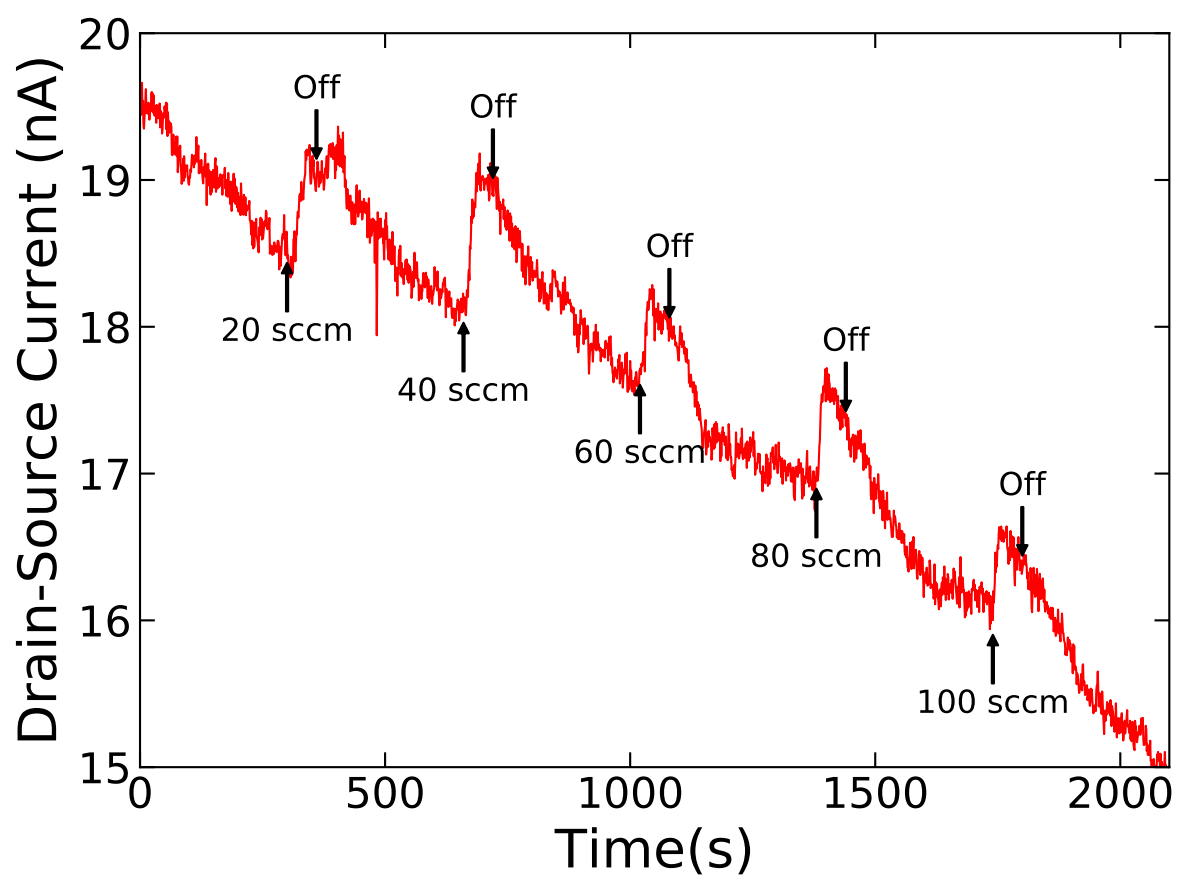

Figure 8.4: Ethanol sensing with a nanowire device. There is a constant flow of $100 \mathrm{sccm}$ through the dilution line, while the vapour line has a flow as labelled on the plot. The device shows a clear, albeit small, response 
In this measurement there is a clear response with increases in the drain-source current when exposed to ethanol vapour. The response is at best a $5 \%$ increase in drain-source current. This corresponds to a sensitivity of 1.05 and is substantially less than that seen in literature $[89,90,92-96]$ where reported sensitivities are generally between 10 and 100 .

\subsubsection{Discussion}

All the measurements in this chapter were taken at room temperature. Ethanol sensors based on $\mathrm{ZnO}$ nanowires [89, 90,92-96] and nanorods [91, 96,97] have been developed in the literature, but typically operate with the device held at elevated temperatures of $200^{\circ} \mathrm{C}-300^{\circ} \mathrm{C}$ [89-97]. Rout et al. [96] demonstrated ethanol sensing with $\mathrm{ZnO}$ nanorods at high temperatures. These nanorods were fabricated in a solution that was then coated and dried on to a substrate to form a dispersed thin film of $\mathrm{ZnO}$ nanorods. At a working temperature of $200^{\circ} \mathrm{C}$, these nanorods achieved a sensitivity of 90 , but at only $50^{\circ} \mathrm{C}$ the sensitivity reduced to 20.

Ethanol sensing measurements are generally not conducted at room temperature and comparison is therefore difficult. In general the higher the temperature, the higher the sensitivity of the $\mathrm{ZnO}$ nanowire/nanorod device [91,94-97], although some papers report a decrease in sensitivity above $300^{\circ} \mathrm{C}[91,95,97]$. As most $\mathrm{ZnO}$ nanorod/nanowire devices only operate at high temperatures, the results presented in this chapter are not conclusive of the ethanol sensing capabilities of these devices. The lack of response from the nanorods, and the minimal response from the nanowires, may be due to the fact that all measurements were taken at room temperature. Further development of the gas rig setup is necessary so that high temperature measurements can be made and this might allow the devices to achieve larger sensitivities.

The response of the nanowires to ethanol in figure 8.4 does not increase as the vapour line flow rate, and hence concentration of ethanol, is increased. This is likely linked to two possible factors. The first factor is that the ethanol vapour might be saturating any detection. The concentration of ethanol in the vapour line is unknown. A very simple approximation can be made using vapour pressures and assuming saturation of the vapour line. Ethanol has a vapour pressure of $5.95 \mathrm{kPa}$ [148] and if we assume that the vapour line is at atmospheric pressure ( $101 \mathrm{kPa}$ [149]), then this gives a concentration of 58,900 ppm. This rough approximation is well above the concentrations measured in the literature (typically $100-1000 \mathrm{pm})$.

Furthermore, Jiaqiang et al. [89] explicitly demonstrated that for a disperse film of $\mathrm{ZnO}$ nanowires (of radius $40-80 \mathrm{~nm}$ and at $300^{\circ} \mathrm{C}$ ) the sensitivity to ethanol vapour starts to saturate at about $2000 \mathrm{ppm}$. Plots of the sensitivity of $\mathrm{ZnO}$ to ethanol vapour concentration by others $[91,95]$ can also be extrapolated and sug- 
gest the saturation of $\mathrm{ZnO}$ at 1000 - 2000 ppm. Future gas sensing measurements will require accurate measurement of the chemical vapour concentrations to ensure that the devices are not saturated. Measurements can then be made in a more suitable range where the concentration of ethanol vapour can be inferred from the data.

The second factor is the ability for the nanowires to return to equilibrium. As described in section 3.2.2, ethanol acts as a reducing agent and reacts with surface oxygen species on the $\mathrm{ZnO}$ surface $[91,93,94]$. This means that in order for the $\mathrm{ZnO}$ to return to its initial state, it must be reoxidised with additional oxygen molecules. Both the vapour and dilution lines are driven with instrument grade $\mathrm{N}_{2}$ and hence very limited oxygen $(<10 \mathrm{ppm})$ is available. This means that between exposures to ethanol vapour, the $\mathrm{ZnO}$ can not necessarily be reoxidised. This is why there appears to be no recovery in figure 8.4 (i.e. a rapid decrease in current) when the vapour line is turned off. In the literature the recovery times (time for the signal to decrease to $90 \%$ of the value) of $\mathrm{ZnO}$ nanowires and nanorods can be quite fast in dry air, with recovery times typically in the order of tens of seconds [89-92,95,96]. Further measurements of the devices used in this thesis should therefore use dry air, rather than $\mathrm{N}_{2}$, as the control gas in the gas rig setup.

\subsection{Conclusion}

In conclusion, both nanorod and nanowire devices were tested as gas sensors for humidity and ethanol vapour. The nanorods showed no response to either, however, the nanowires had a clear response to ethanol and possibly a very small response to humidity. These results show that in principle the nanowires might be viable as gas sensors, but improvements to the gas sensing setup (heating of the device, accurate gas concentration measurement and refitting of the gas rig with compressed air) will be necessary to achieve any quantitative results. 


\section{Chapter 9}

\section{Conclusions}

This chapter presents an overview of the key results of this thesis, followed by suggestions for further work in developing the $\mathrm{ZnO}$ nanorod and nanowire devices as sensors.

\subsection{Summary}

$\mathrm{ZnO}$ nanowires were fabricated using a hydrothermal method and used as the semiconductor in field effect transistors. Two device types were fabricated called nanorods and nanowires (the two terms are often used interchangeably in the literature, but generally nanorods are bigger than nanowires [132]). The nanorod devices consist of large $\mathrm{ZnO}$ crystals of $400 \mathrm{~nm}$ in diameter, while the nanowire devices consist of nanorods that have much thinner nanowires, of a diameter of $20 \mathrm{~nm}$, growing off their surfaces.

The devices were optimised for sensing by promoting the growth of exclusively lateral nanorods. This removed any superfluous vertical nanorods that do not make an electrical connection between the drain and source. Furthermore the vertical nanorods might penetrate the encapsulation layer, causing undesirable leakage currents.

Lateral growth was achieved by growing $\mathrm{ZnO}$ nanorods from predeposited seed layers of $\mathrm{ZnO}$, capped with a layer of titanium. The capping layer constrains the nucleation of the nanorods to growth from the edge of the $\mathrm{ZnO} / \mathrm{Ti}$ stack. Acid was then used to etch the $\mathrm{ZnO}$ layer, leaving an overhang of Ti. This acted as a mechanical barrier to the growth of the nanorods and constricted them to lateral growth. Two acids were trialled; $1 \mathrm{mM} \mathrm{H}_{3} \mathrm{PO}_{4}$ and $0.89 \mathrm{mM} \mathrm{HCl}$. Both acids were diluted such that they were the same $\mathrm{pH}$ (3.05) and an SEM was used to image the overhanging $\mathrm{Ti}$ layer. The etch rate of the two acids was indistinguishable and approximately $25 \mathrm{nms}^{-1}$. The maximum angular deviation of the nanorod structures from the horizontal was also imaged with the SEM. An etch rate of 20 s with $1 \mathrm{mM} \mathrm{H}_{3} \mathrm{PO}_{4}$ or $0.89 \mathrm{mM} \mathrm{HCl}$ was deemed to produce the most lateral nanorod structures, without collapse of the Ti overhang.

The nanorod and nanowire devices were then characterised as FETs. The devices were characterised as FETs both dry and with a liquid gate dielectric. Multiple gating setups were used, including backgating through the $\mathrm{Si} / \mathrm{SiO}_{2}$ and topgating with a $\mathrm{Ag} / \mathrm{AgCl}$ electrode inserted into the liquid. The nanorod samples 
showed negligible field dependence when dry, but once wet they achieved on/off ratios of $10^{3}$. The nanowires did show field dependence both when dry and when wet, with slightly better on/off ratios of $10^{4}$. The nanowires were deemed to be the more promising device type and did not become highly conductive when hardbaked (which is necessary for the functionalisation). The nanowires were further tested in a variety of other liquids suitable for sensing applications.

Attempts were made to functionalise the nanowire devices with aptamers. A silane linker group GOPS was linked to the $\mathrm{ZnO}$ surface. However, once exposed to aptamers in tris buffer, the nanowires dissolved. The rate of decay of the nanowires in tris buffer was measured and loosely fitted with an exponential decay. The decay rate was measured to be $-0.0195 \mathrm{~s}^{-1}$, giving a current half life of $36 \mathrm{~s}$. Nanowires that had not been functionalsied, however, did not show the same rapid decay. This suggests that the functionalisation process is resulting in some major chemical or structural change in the nanowires that makes them more reactive with tris buffer.

Both the nanorod and nanowire devices were tested as chemiresistor gas sensors for the detection of humidity and ethanol vapour. The nanorod devices showed no response to either humidity or ethanol. The nanowires showed a negligible response to humidity and a small response to ethanol vapour. Further development of the gas rig setup will be necessary to see the true capabilities of these devices.

\subsection{Future Work}

In this section a number of ideas are suggested for future work on developing the $\mathrm{ZnO}$ nanowires as biosensors and gas sensors.

\subsubsection{Biosensors}

A number of issues regarding the stability of the $\mathrm{ZnO}$ nanowires were identified in this thesis. First, in section 6.3, the $\mathrm{ZnO}$ nanowires and nanorods showed notable hysteresis between measurements when gated in DI water. A practical device would have to have reproducible results and the hysteresis effects may hinder efforts to achieve this. Second, the nanowires rapidly dissolved in tris buffer during the functionalisation process.

As discussed in section 7.3, the degradation of $\mathrm{ZnO}$ nanowires in biological fluids is thought to originate from the formation of $\mathrm{ZnO}(\mathrm{OH})_{4}{ }^{2-}$ complexes at defects on the $\mathrm{ZnO}$ surface [147]. As the unfuntionalised $\mathrm{ZnO}$ nanowires were relatively stable in tris buffer, it may be useful to identify which stage in the GOPS modification procedure is causing the insatiability. Photoluminescence spectra could be 
used (as in [147]) to identify any changes in the number of defects or impurities in the $\mathrm{ZnO}$ nanowires between each step. Energy-dispersive X-ray spectroscopy and X-ray diffraction measurements may also prove useful to investigate changes in the chemical and crystal properties of the nanowires. In addition these same tools could be used to investigate the increase in conductivity of the nanorods when hardbaked (as described in section 6.1.3).

A possible solution to the degradation issues is to passivate the surface of the $\mathrm{ZnO}$ nanowires. Passivation involves coating the nanowires with a thin film of dielectric. There are a number of examples of passivation layers used in $\mathrm{ZnO} F E T s$ including using $\mathrm{SiO}_{2}$ [70], a bilayer of $\mathrm{SiO}_{2} / \mathrm{Si}_{3} \mathrm{~N}_{4}$ [67] and layer of polymethyl methacrylate (PMMA) $[65,102,104,150]$. The passivation layer has two effects. First passivation layers can improve the FET characteristics of $\mathrm{ZnO}$ nanowires. For example, Chang et al. [67] characterised FETs fabricated from single ZnO nanowires (diameter $90 \mathrm{~nm}$, synthesised using chemical vapour deposition) before and after passivation. Pasivation was achieved by depositing a bilayer of $100 \mathrm{~nm} \mathrm{SiO}{ }_{2}$ and $50 \mathrm{~nm} \mathrm{SiN} \mathrm{on} \mathrm{top} \mathrm{of} \mathrm{the} \mathrm{nanowires.} \mathrm{The} \mathrm{passivation} \mathrm{resulted} \mathrm{in}$ an increase in the on/off ratio from $10^{3}$ to $10^{4}$ and a massive reduction in subthreshold swing from $3 \mathrm{~V} /$ decade to $150 \mathrm{mV} /$ decade.

Second, the passivation layer provides a physical barrier between the nanowires and the environment. The adsorption or desorption of molecules is therefore inhibited and the associated effects such as atmospheric dependence and hysteresis can be stopped. Song et al. [65] showed that the transfer characteristics of single $\mathrm{ZnO}$ nanowire FETs differed greatly when in vacuum to when in air. Upon passivation with PMMA, however, there were no longer any notable differences. Furthermore, Sohn et al. [104] showed that hysteresis in single ZnO nanowire FETs could be reduced by passivation with PMMA.

In order to further develop the $\mathrm{ZnO}$ nanowires as a biosensor, a thin passivation layer could be used to seperate and protect the nanowires from any buffer solutions. The passivation material could also be selected so that it could be functionalsied with GOPS and, hence aptamers. Examples include alumina $\left(\mathrm{Al}_{2} \mathrm{O}_{3}\right)$ [151], $\mathrm{SiO}_{2}$ [151] or $\mathrm{SiN}$ [151]. If a very thin film (e.g. $<10 \mathrm{~nm}$ ) was coated onto the nanowires, the surface of this layer could then be functionalised. The electrostatic effect of aptamer binding should be large enough to penetrate the passivation layer and interact with the $\mathrm{ZnO}$ nanowires. In this way the $\mathrm{ZnO}$ nanowires can still be used in an aptamer based biosensor, while protected from any chemical reactions with the buffer.

Another possibility is to use a metal-semiconductor field effect transistor (MESFET) structure. MESFETs are a variant of an FET that place a metal gate directly on top of the semiconductor. The metal top gate layer is very thin (i.e. a few $\mathrm{nm}$ ) and forms a Schottky contact with the semiconductor (for more on MESFETs see [12]). MESFETs fabricated from $\mathrm{ZnO}$ thin films have been developed in literature $[152,153]$ and can achieve on/off ratios of $1.2 \times 10^{8}$ across a very narrow gate voltage range of -1.5 to $0 \mathrm{~V}_{G}$ [153]. 
MESFET biosensors have been developed with carbon nanotubes (CNTs) as demonstrated by $\mathrm{Oh}$ et al. [154,155]. They showed that a $1 \mathrm{~nm} / 5 \mathrm{~nm}$ chrome/gold topgate could be deposited onto CNTs and successfully functionalised with thiol terminated aptamers [154].In another paper, Oh et al. [155] demonstrated the sensing of horse radish peroxidase (HRP) in phosphate buffered saline, on a similar CNT MESFET (the gate was functionalised with an antibody that binds to HRP) and achieved sensing between $1 \mathrm{pg} / \mathrm{ml}$ and 10 $\mathrm{ng} / \mathrm{ml}$ of HRP.

The MESFET structure could be used to enclose the $\mathrm{ZnO}$ nanowires with the metal topgate, in a similar manner to the passivation layer described above. If gold was used (as demonstrated in ZnO thin film MESFETs [152,153]) for the topgate, then it could be functionalised with thiol terminated aptamers and used as a bisoensor without the risk of dissolving in buffer solution.

\subsubsection{Gas Sensors}

In chapter 8 the nanowire devices showed some response to ethanol vapour, but as discussed in sections 8.1.3 and 8.2.3, there were a number of issues with the experimental setup that could be improved.

Most ethanol vapour sensors based on $\mathrm{ZnO}$ nanowires only operate at elevated temperatures of $200^{\circ} \mathrm{C}-300^{\circ} \mathrm{C}$ [89-97] and in general the higher the temperature, the higher the sensitivity of the $\mathrm{ZnO}$ nanowire/nanorod device [91,94-97]. All the measurements presented in chapter 8 , however, were taken at room temperature. It would therefore be of interest to refit the gas sensing rig with a heater so that the devices can be held at elevated temperatures. Greater sensitivities might be achievable with the nanowires, and perhaps even the nanorods, when heated.

Secondly, there is the issue regarding the concentration of the chemical vapours in the vapour line. In figure 8.4 the sensitivity of the $\mathrm{ZnO}$ nanowire device does not increase as the vapour line flow rate, and hence concentration of ethanol, is increased. Assuming saturation of the ethanol vapour at a vapour pressure of $5.95 \mathrm{kPa}$ [148] and a total atmospheric pressure of $101 \mathrm{kPa}$ [149], the vapour line would have a concentration of $58,900 \mathrm{ppm}$. This is well above the saturation limit of $\mathrm{ZnO}$ as measured in the literature $[89,91,95]$. Future gas sensing measurements may require the use of a control sensor that can accurately measure the concentration of ethanol vapour concentration. This would allow measurements to be made in a concentration range where the $\mathrm{ZnO}$ nanowires are not saturated and the exact vapour concentration can be inferred from the data.

Finally, the gas rig setup currently uses $\mathrm{N}_{2}$ gas as the control gas in both the vapour and dilution lines. The gas sensing mechanism relies on the desorption, adsorption and reactions of oxygen species on the $\mathrm{ZnO}$ surface $[83,89,91,93]$. The instrument grade $\mathrm{N}_{2}$ has less than $10 \mathrm{ppm}$ of $\mathrm{O}_{2}$ and hence the reoxidation of 
the $\mathrm{ZnO}$ nanowire surface between exposure to chemical vapours is very limited. Sequential exposures may result in a signal, but the sensitivity is offset by the previous exposure. In order to allow the device to reset to an equilibrium, the gas rig should be refit with a control gas containing $\mathrm{O}_{2}$, (i.e. air).

\subsection{Final Word}

In this thesis hydrothermally grown lateral $\mathrm{ZnO}$ nanorods and nanowires have been used in field effect transistors and attempts were made to apply them as biosensors and gas sensors. These attempts were unsuccessful and a number of issues have been identified. There are still many possible options for further development and improvement of these devices. 


\section{Appendix A}

\section{Reproducibility of Devices}

This appendix will briefly discuss the reproducibility of the transfer characteristics between devices. Only the nanowire devices in the backgated configuration when dry will be covered. As discussed in section 6.3, both the nanorods and nanowires show substantial hysteresis between measurements in liquid conditions. Direct comparison of electrical data when immersed in liquid is therefore not necessarily valid. Furthermore, comparison of the transfer characteristics of dry nanorod devices is of little interest due to their negligible field dependence.

As described in section 6.2.1, the nanowire devices showed a reasonable degree of variability. The nanowires grow off the large nanorods and so a small change in nanorod structure leads to a large change in the nanowire structure.

It is assumed that the variation in number and density of nanowires is the primary cause of the variation in transfer characteristics of devices. Figure A.1 shows a plot of on and off currents, as well as the on/off ratio, for all the measured nanowire devices. There is a wide distribution of on/off ratios ranging over at least five orders of magnitude. It is notable that the devices with a low on/off ratio generally have a high off current, while the on current is comparable to devices with a high on/off ratio.

In most devices with an on/off ratio less than 100 there are large $\mathrm{ZnO}$ nanorods or debris in the channel of the device. As described in section 6.1.1, the nanorods are conductive and have negligible field dependence. Therefore the current through the device is offset by any base current through the rods, leading to a large off current and poor on/off ratio. An example of a nanowire device with nanorods in the channel is shown in figure A.2.

Clearly improvements could be made to the fabrication process to reduce the amount of debris that forms on the devices. The formation of $\mathrm{ZnO}$ debris is most likely due to nucleation on imperfections or other forms of debris present on the substrate surface before hydrothermal growth. The fabrication of devices was completed with as much care and cleanliness as possible, however any improvements in this regard could help reduce the formation of unwanted $\mathrm{ZnO}$ debris or nanorods. In turn this could lead to more reproducible nanowire devices, with more consistent transfer characteristics. 


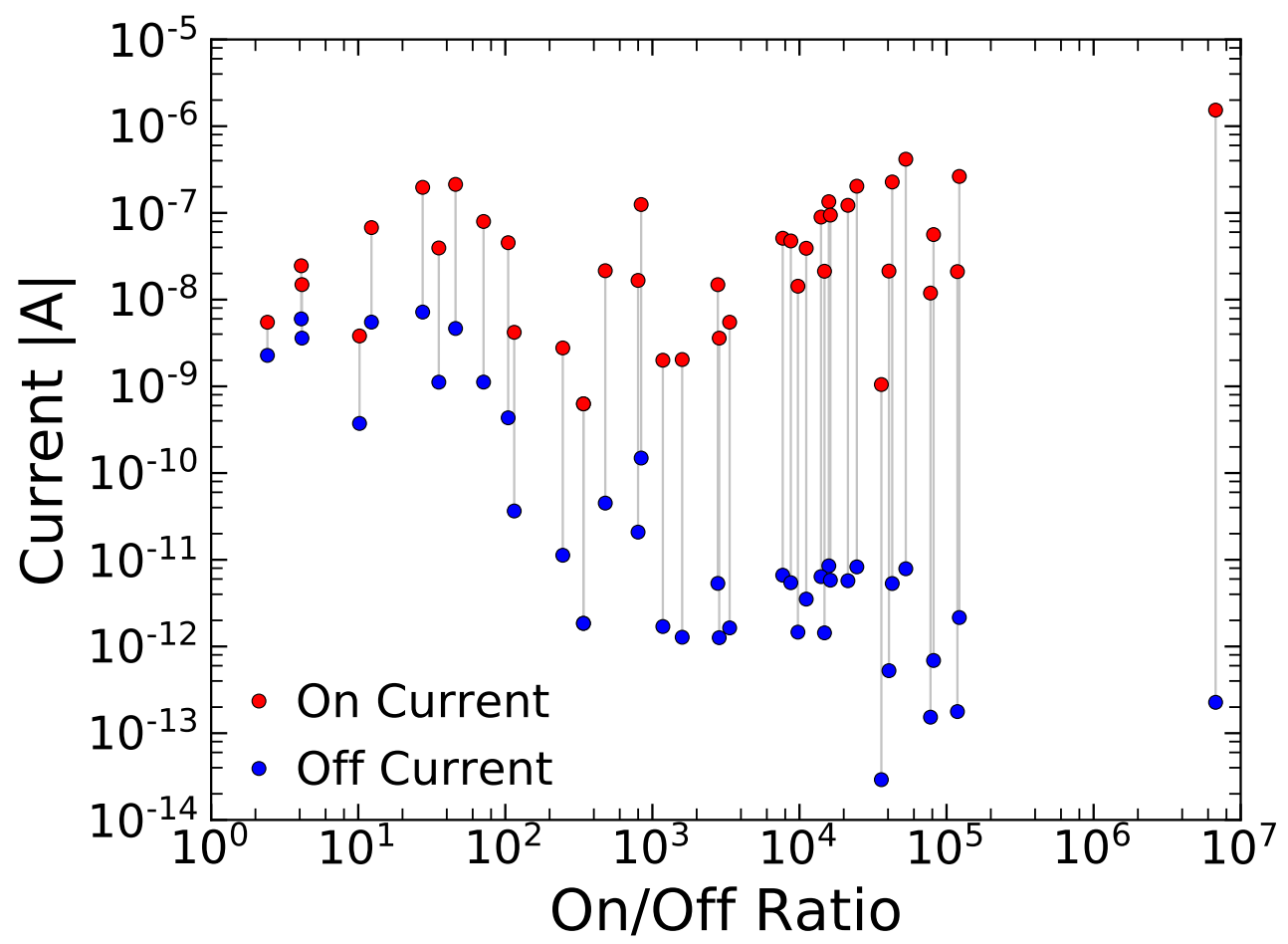

Figure A.1: A plot of on/off ratios for the nanowire devices measured for this thesis. The vertical lines link the on and off currents for each device. All devices were driven at $0.5 \mathrm{~V}_{D S}$ and the gate voltage was swept from $-10 \mathrm{~V}$ to $10 \mathrm{~V}$ and back.

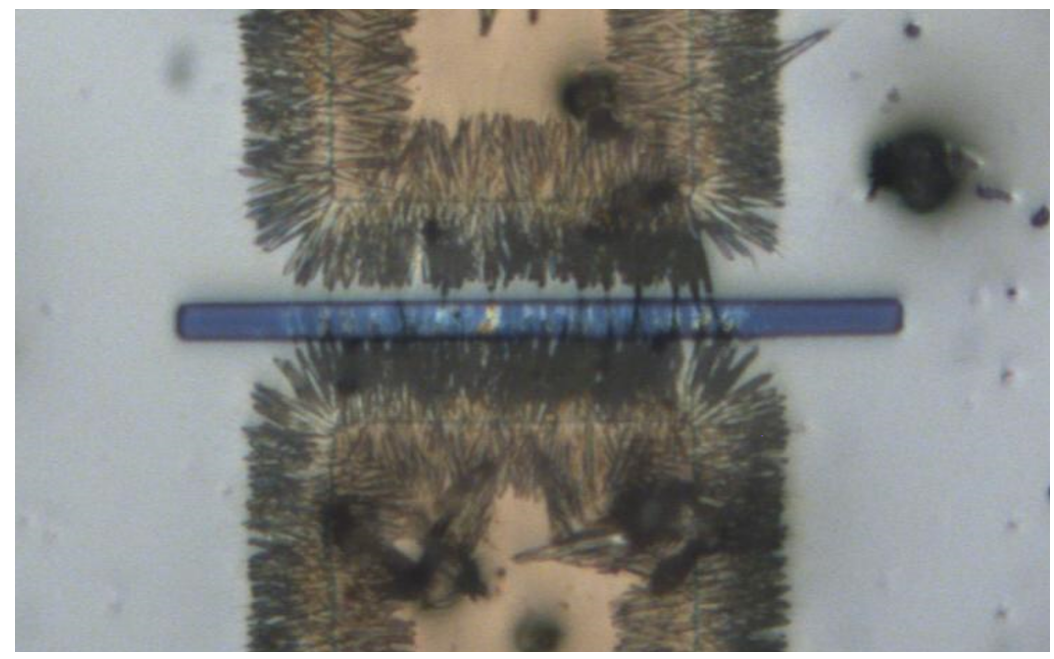

Figure A.2: A nanowire sample with rods crossing the channel. The nanowires are the faint white lines, while the larger dark lines on the right are nanorods crossing the channel. There also appears to be a piece of $\mathrm{ZnO} / \mathrm{Ti}$ (beige colour) in the middle of the channel which has not been properly lifted off. This device can be contrasted with the device shown in figure 5.12 . 


\section{List of Abbreviations}

$\begin{array}{ll}\text { DI } & \text { Deionised } \\ \text { FET } & \text { Field Effect Transistor } \\ \text { HMT } & \text { Hexamethylenetetraamine } \\ \text { IPA } & \text { Isopopyl Alcohol } \\ \text { mM } & \text { Millimolar or Millimols per Litre } \\ \text { NH } & \\ \text { PBS } & \text { Ammonia } \\ \text { PCB } & \text { Phosphate-Buffered Saline } \\ \text { PDMS } & \text { Printed Circuit Board } \\ \text { PEI } & \text { Polydimethylsiloxane } \\ \text { PGMEA } & \text { Polyethylenimine } \\ \text { ppm } & \text { Propylene Glycol Methyl Ether Acetate } \\ \text { RPM } & \text { Parts Per Million } \\ \text { Sccm } & \text { Revolutions Per Minute } \\ \text { SEM } & \text { Standard Cubic Centimetres } \\ \text { Tris } & \text { Scanning Electron Microscope } \\ \text { VUW } & \text { Trisaminomethane } \\ \end{array}$


REFERENCES 


\section{References}

[1] Edgar T Wherry. Radio detector minerals. Mineralogical Society of America, 10:28-31, 1925.

[2] CW Bunn. The lattice-dimensions of zinc oxide. Proceedings of the Physical Society, 47(5):835, 1935.

[3] E Mollwo. Die wirkung von wasserstoff auf die leitfähigkeit und lumineszenz von zinkoxydkristallen. Zeitschrift für Physik A Hadrons and Nuclei, 138(3):478-488, 1954.

[4] AR Hutson. Piezoelectricity and conductivity in zno and cds. Physical Review Letters, 4(10):505, 1960.

[5] Klaus Ellmer, Andreas Klein, and Bernd Rech. ZnO and Its Applications, Basics and Applications in Thin Film Solar Cells. Springer, 2008.

[6] Ü Özgür, Ya I Alivov, Chunli Liu, A Teke, MAn Reshchikov, S Doğan, VCSJ Avrutin, S-J Cho, and H Morkoc. A comprehensive review of zno materials and devices. Journal of applied physics, 98(4):11, 2005.

[7] Özgür Ümit, Daniel Hofstetter, and Hadis Morkoc. Zno devices and applications: a review of current status and future prospects. Proceedings of the IEEE, 98(7):1255-1268, 2010.

[8] Ümit Özgür and Hadis Morkoç. Zinc Oxide, Fundammenatls, Materials and Device Technology. Wiley, 2009.

[9] John F Wager. Transparent electronics. science, 300(5623):1245-1246, 2003.

[10] Philip G Neudeck, Robert S Okojie, and Liang-Yu Chen. High-temperature electronics-a role for wide bandgap semiconductors? Proceedings of the IEEE, 90(6):1065-1076, 2002.

[11] Zhong Lin Wang. Nanostructures of zinc oxide. Materials today, 7(6):26-33, 2004.

[12] SM Sze and KK Ng. Physics of Semiconductor Devices, Third Edition. John Wiley and Sons, 2006.

[13] Brett Lee Allen, Padmakar D Kichambare, and Alexander Star. Carbon nanotube field-effect-transistor-based biosensors. Advanced Materials, 19(11):1439-1451, 2007. 
[14] Adam K Wanekaya, Wilfred Chen, Nosang V Myung, and Ashok Mulchandani. Nanowire-based electrochemical biosensors. Electroanalysis, 18(6):533-550, 2006.

[15] Guo-Jun Zhang and Yong Ning. Silicon nanowire biosensor and its applications in disease diagnostics: a review. Analytica Chimica Acta, 749:1-15, 2012.

[16] Sunil K. Arya. Recent advances in $\mathrm{ZnO}$ nanostructures and thin films for biosensor applications. Analytica Chimica Acta, 2012.

[17] Saraju P Mohanty and Elias Kougianos. Biosensors: a tutorial review. Ieee Potentials, 25(2):35-40, 2006.

[18] Jeong-O Lee, Hye-Mi So, Eun-Kyoung Jeon, Hyunju Chang, Keehoon Won, and Yong Hwan Kim. Aptamers as molecular recognition elements for electrical nanobiosensors. Analytical and bioanalytical chemistry, 390(4):1023$1032,2008$.

[19] Christian Leiterer, Barbara Seise, Irma Slowik, Gerald Brönstrup, Raphael Niepelt, Karina Weber, Carsten Ronning, Silke Christiansen, and Wolfgang Fritzsche. DNA hybridization assay at individual, biofunctionalized zinc oxide nanowires. Journal of Biophotonics, 6(2):143-147, 2013.

[20] B. D. Yao, Y. F. Chan, and N. Wang. Formation of ZnO nanostructures by a simple way of thermal evaporation. Applied Physics Letters, 81(4):757-759, 2002.

[21] Ye Sun, Gareth M. Fuge, and Michael N R Ashfold. Growth of aligned ZnO nanorod arrays by catalyst-free pulsed laser deposition methods. Chemical Physics Letters, 396(1-3):21-26, 2004.

[22] Won Il Park, Jin Suk Kim, Gyu-Chul Yi, MH Bae, and H-J Lee. Fabrication and electrical characteristics of high-performance zno nanorod field-effect transistors. Applied Physics Letters, 85(21):5052-5054, 2004.

[23] Jih-Jen Wu and Sai-Chang Liu. Catalyst-Free Growth and Characterization of ZnO Nanorods. The Journal of Physical Chemistry B, 106(37):9546-9551, 2002.

[24] P. Yang, H. Yan, S. Mao, R. Russo, J. Johnson, R. Saykally, N. Morris, J. Pham, R. He, and H. J. Choi. Controlled growth of $\mathrm{ZnO}$ nanowires and their optical properties. Advanced Functional Materials, 12(5):323-331, 2002.

[25] Lionel Vayssieres. Growth of arrayed nanorods and nanowires of $\mathrm{ZnO}$ from aqueous solutions. Advanced Materials, 15(5):464-466, 2003.

[26] Lei Li, Shuming Yang, Qingjie Hu, Chenying Wang, Fengxia Zhao, and Zhuangde Jiang. The zno nanwire controllable synthesis and its optical properties. In Nanotechnology (IEEE-NANO), 2012 12th IEEE Conference on, pages 1-5. IEEE, 2012. 
[27] Min Guo, Peng Diao, and Shengmin Cai. Hydrothermal growth of perpendicularly oriented $\mathrm{ZnO}$ nanorod array film and its photoelectrochemical properties. Applied Surface Science, 249(1-4):71-75, 2005.

[28] Min Guo, Peng Diao, and Shengmin Cai. Hydrothermal growth of wellaligned $\mathrm{ZnO}$ nanorod arrays: Dependence of morphology and alignment ordering upon preparing conditions. Journal of Solid State Chemistry, 178(6):1864-1873, 2005.

[29] Sunandan Baruah and Joydeep Dutta. Hydrothermal growth of zno nanostructures. Science and Technology of Advanced Materials, 10(1):013001, 2009.

[30] Michael NR Ashfold, Rachel P Doherty, N George Ndifor-Angwafor, D Jason Riley, and Ye Sun. The kinetics of the hydrothermal growth of zno nanostructures. Thin Solid Films, 515(24):8679-8683, 2007.

[31] Abhilash Sugunan, Hemant C Warad, Mats Boman, and Joydeep Dutta. Zinc oxide nanowires in chemical bath on seeded substrates: role of hexamine. Journal of Sol-Gel Science and Technology, 39(1):49-56, 2006.

[32] Bin Liu, Hua Chun Zeng, Bin Liu, and Hua Chun Zeng. Hydrothermal Synthesis of ZnO Nanorods in the Diameter Regime of $50 \mathrm{~nm} \mathrm{Hy-}$ drothermal Synthesis of $\mathrm{ZnO}$ Nanorods in the Diameter Regime of $50 \mathrm{~nm}$. 125(March):4430-4431, 2003.

[33] J. B. Baxter, A. M. Walker, K. Van Ommering, and E. S. Aydil. Synthesis and characterization of $\mathrm{ZnO}$ nanowires and their integration into dyesensitized solar cells. Nanotechnology, 17(11), 2006.

[34] Min Guo, Peng Diao, Xindong Wang, and Shengmin Cai. The effect of hydrothermal growth temperature on preparation and photoelectrochemical performance of $\mathrm{ZnO}$ nanorod array films. Journal of Solid State Chemistry, 178(10):3210-3215, 2005.

[35] Jijun Qiu, Xiaomin Li, Fuwei Zhuge, Xiaoyan Gan, Xiangdong Gao, Weizhen He, Se-Jeong Park, Hyung-Kook Kim, and Yoon-Hwae Hwang. Solution-derived $40 \mathrm{~m}$ vertically aligned $\mathrm{ZnO}$ nanowire arrays as photoelectrodes in dye-sensitized solar cells. Nanotechnology, 21(19):195602, 2010.

[36] Jason B. Baxter and Eray S. Aydil. Nanowire-based dye-sensitized solar cells. Applied Physics Letters, 86(5):1-3, 2005.

[37] Y. F. Hsu, Y. Y. Xi, A. B. Djurišić, and W. K. Chan. ZnO nanorods for solar cells: Hydrothermal growth versus vapor deposition. Applied Physics Letters, 92(13):2006-2009, 2008.

[38] C. Y. Jiang, X. W. Sun, K. W. Tan, G. Q. Lo, A. K.K. Kyaw, and D. L. Kwong. High-bendability flexible dye-sensitized solar cell with a nanoparticlemodified ZnO-nanowire electrode. Applied Physics Letters, 92(14):90-93, 2008 . 
[39] Seung Hwan Ko, Daeho Lee, Hyun Wook Kang, Koo Hyun Nam, Joon Yeob Yeo, Suk Joon Hong, Costas P. Grigoropoulos, and Hyung Jin Sung. Nanoforest of hydrothermally grown hierarchical $\mathrm{ZnO}$ nanowires for a high efficiency dye-sensitized solar cell. Nano Letters, 11(2):666-671, 2011.

[40] SB Zhang, S-H Wei, and Alex Zunger. Intrinsic n-type versus p-type doping asymmetry and the defect physics of zno. Physical Review B, 63(7):075205, 2001.

[41] Vitaliy Avrutin, Donald J Silversmith, and Hadis Morkoç. Doping asymmetry problem in zno: current status and outlook. Proceedings of the IEEE, 98(7):1269-1280, 2010.

[42] Claus F. Klingshirn, Bruno K. Meyer, Andreas Waag, Axel Hoffmann, and Jean Geurts. Zinc Oxide, From Fundammenatl Properties Towards Novel Applications. Springer, 2010.

[43] Charles Kittel. Introduction to Solid State Physics, Second Edition. John Wiley and Sons, 1963.

[44] Conor Burke-Govey. ZnO nanowires and their application in field-effect transistors. PhD thesis, Victroia University of Wellington, 2017.

[45] Lukas Schmidt-Mende and Judith L MacManus-Driscoll. Znonanostructures, defects, and devices. Materials today, 10(5):40-48, 2007.

[46] Lori E Greene, Matt Law, Joshua Goldberger, Franklin Kim, Justin C Johnson, Yanfeng Zhang, Richard J Saykally, and Peidong Yang. Lowtemperature wafer-scale production of zno nanowire arrays. Angewandte Chemie International Edition, 42(26):3031-3034, 2003.

[47] Kuanfei Liu, Weibing $\mathrm{Wu}$, Baolong Chen, Xiaodong Chen, and Nannan Zhang. Continuous growth and improved pl property of zno nanoarrays with assistance of polyethylenimine. Nanoscale, 5(13):5986-5993, 2013.

[48] WJ Gibbs. On the equilibrium of heterogeneous substances. Transactions of the Connecticut Academy of Arts and Sciences, 3:108-524, 1878.

[49] James J De Yoreo and Peter G Vekilov. Principles of crystal nucleation and growth. Reviews in mineralogy and geochemistry, 54(1):57-93, 2003.

[50] Zhong Lin Wang. Zinc oxide nanostructures: growth, properties and applications. Journal of Physics: Condensed Matter, 16(25):R829, 2004.

[51] Wen-Jun Li, Er-Wei Shi, Wei-Zhuo Zhong, and Zhi-Wen Yin. Growth mechanism and growth habit of oxide crystals. Journal of crystal growth, 203(1):186-196, 1999.

[52] Jaebum Joo, Brian Y Chow, Manu Prakash, Edward S Boyden, and Joseph M Jacobson. Face-selective electrostatic control of hydrothermal zinc oxide nanowire synthesis. Nature materials, 10(8):596-601, 2011. 
[53] Ying Zhou, Weibing $\mathrm{Wu}$, Guangda $\mathrm{Hu}$, Haitao $\mathrm{Wu}$, and Shougang Cui. Hydrothermal synthesis of zno nanorod arrays with the addition of polyethyleneimine. Materials Research Bulletin, 43(8):2113-2118, 2008.

[54] Takuya Fujimoto and Kunio Awaga. Electric-double-layer field-effect transistors with ionic liquids. Physical Chemistry Chemical Physics, 15(23):89839006, 2013.

[55] Eric Stern, Robin Wagner, Fred J Sigworth, Ronald Breaker, Tarek M Fahmy, and Mark A Reed. Importance of the debye screening length on nanowire field effect transistor sensors. Nano letters, 7(11):3405-3409, 2007.

[56] Jacob N Israelachvili. Intermolecular and Surface Forces, Third Edition. Academic Press, 2011.

[57] S. Thiemann, S. Sachnov, S. Porscha, P. Wasserscheid, and J. Zaumseil. Ionic liquids for electrolyte-gating of $\mathrm{ZnO}$ field-effect transistors. Journal of Physical Chemistry C, 116(25):13536-13544, 2012.

[58] Kenzo Maehashi, Taiji Katsura, Kagan Kerman, Yuzuru Takamura, Kazuhiko Matsumoto, and Eiichi Tamiya. Label-free protein biosensor based on aptamer-modified carbon nanotube field-effect transistors. Analytical Chemistry, 79(2):782-787, 2007.

[59] Stefan Duhr and Dieter Braun. Why molecules move along a temperature gradient. Proceedings of the National Academy of Sciences, 103(52):1967819682, 2006.

[60] Hongtao Yuan, Hidekazu Shimotani, Atsushi Tsukazaki, Akira Ohtomo, Masashi Kawasaki, and Yoshihiro Iwasa. High-density carrier accumulation in $\mathrm{ZnO}$ field-effect transistors gated by electric double layers of ionic liquids. Advanced Functional Materials, 19(7):1046-1053, 2009.

[61] Andy Burrows, Andy Parsons, Gareth Price, Gwen Pilling, and John Holman. Chemistry ${ }^{3}$. Oxford, 2009.

[62] D R Lide. Handbook of Chemsitry and Physics, Eighty-Eighth Edition. CRC Press, 2008.

[63] Adelmo Ortiz-Conde, FJ Garcia Sánchez, Juin J Liou, Antonio Cerdeira, Magali Estrada, and Y Yue. A review of recent mosfet threshold voltage extraction methods. Microelectronics Reliability, 42(4):583-596, 2002.

[64] Dieter K Schroder. Seminconductor material and Device Characterization, Third Edition. John Wiley and Sons, 2006.

[65] Sunghoon Song, Woong Ki Hong, Soon Shin Kwon, and Takhee Lee. Passivation effects on $\mathrm{ZnO}$ nanowire field effect transistors under oxygen, ambient, and vacuum environments. Applied Physics Letters, 92(26):2006-2009, 2008. 
[66] Josh Goldberger, Donald J Sirbuly, Matt Law, and Peidong Yang. Zno nanowire transistors. The Journal of Physical Chemistry B, 109(1):9-14, 2005.

[67] Pai-Chun Chang, Zhiyong Fan, Chung-Jen Chien, Daniel Stichtenoth, Carsten Ronning, and Jia Grace Lu. High-performance $\mathrm{ZnO}$ nanowire field effect transistors. Applied Physics Letters, 89(13):133113, 2006.

[68] C Opoku, AS Dahiya, F Cayrel, G Poulin-Vittrant, D Alquier, and N Camara. Fabrication of field-effect transistors and functional nanogenerators using hydrothermally grown zno nanowires. RSC Advances, 5(86):6992569931, 2015.

[69] Charles Opoku, Abhishek Singh Dahiya, Christopher Oshman, Christophe Daumont, Frederic Cayrel, Guylaine Poulin-Vittrant, Daniel Alquier, and Nicolas Camara. Fabrication of high performance field-effect transistors and practical schottky contacts using hydrothermal zno nanowires. Nanotechnology, 26(35):355704, 2015.

[70] Hyeong Jin Kim, Chul Ho Lee, Dong Wook Kim, and Gyu Chul Yi. Fabrication and electrical characteristics of dual-gate $\mathrm{ZnO}$ nanorod metal-oxide semiconductor field-effect transistors. Nanotechnology, 17(11):1-6, 2006.

[71] Po-Yu Yang, Jyh-Liang Wang, Wei-Chih Tsai, Shui-Jinn Wang, Jia-Chuan Lin, I-Che Lee, Chia-Tsung Chang, and Huang-Chung Cheng. Photoresponse of hydrothermally grown lateral zno nanowires. Thin Solid Films, 518(24):7328-7332, 2010.

[72] Yong Kyu Park, Han Seok Choi, Jin-Hwan Kim, Jeong-Hyun Kim, and Yoon-Bong Hahn. High performance field-effect transistors fabricated with laterally grown ZnO nanorods in solution. Nanotechnology, 22(18):185310, 2011.

[73] Seung H. Ko, Inkyu Park, Heng Pan, Nipun Misra, Matthew S. Rogers, Costas P. Grigoropoulos, and Albert P. Pisano. ZnO nanowire network transistor fabrication on a polymer substrate by low-temperature, all-inorganic nanoparticle solution process. Applied Physics Letters, 92(15), 2008.

[74] Zhiyong Fan, Dawei Wang, Pai-Chun Chang, Wei-Yu Tseng, and Jia G Lu. Zno nanowire field-effect transistor and oxygen sensing property. Applied Physics Letters, 85(24):5923-5925, 2004.

[75] Baoquan Sun and Henning Sirringhaus. Solution processed Zinc Oxide field effect transistors based on self-assembly aof colloidal nanorods. Nano Letters, 5(12):0-5, 2005.

[76] Frank Jones, François Léonard, A. Alec Talin, and Nelson S. Bell. Electrical conduction and photoluminescence properties of solution-grown $\mathrm{ZnO}$ nanowires. Journal of Applied Physics, 102(1), 2007. 
[77] Stefan Thiemann, Mathias Gruber, Irina Lokteva, Johannes Hirschmann, Marcus Halik, and Jana Zaumseil. High-Mobility ZnO Nanorod Field-E ff ect Transistors by Self- Alignment and Electrolyte-Gating. ACS Applied Materials \& Interfaces, 5(5):1656-1662, 2013.

[78] Daniel Kalblein, $\mathrm{R}$ Thomas Weitz, H Jens Bottcher, Frederik Ante, Ute Zschieschang, Klaus Kern, and Hagen Klauk. Top-gate zno nanowire transistors and integrated circuits with ultrathin self-assembled monolayer gate dielectric. Nano letters, 11(12):5309-5315, 2011.

[79] Daniel Kälblein, Hyeyeon Ryu, Frederik Ante, Bernhard Fenk, Kersten Hahn, Klaus Kern, and Hagen Klauk. High-performance ZnO nanowire transistors with aluminum top-gate electrodes and naturally formed hybrid self-assembled monolayer/AlOx gate dielectric. ACS Nano, 8(7):68406848, 2014.

[80] Jae Eun Jang, Seung Nam Cha, Tim P Butler, Jung In Sohn, Jung Woo Kim, Young Wan Jin, Gehan AJ Amaratunga, Jae Eun Jung, and Jong Min Kim. A characterization study of a nanowire-network transistor with various channel layers. Advanced materials, 21(41):4139-4142, 2009.

[81] Vivek Pachauri, Alexis Vlandas, Klaus Kern, and Kannan Balasubramanian. Site-specific self-assembled liquid-gated $\mathrm{ZnO}$ nanowire transistors for sensing applications. Small, 6(4):589-594, 2010.

[82] Hanyue Xheng. Carbon nanotube thin film transistor platforms for small molecule aptasensors. PhD thesis, Victroia University of Wellington, 2016.

[83] F Chaabouni, M Abaab, and B Rezig. Metrological characteristics of zno oxygen sensor at room temperature. Sensors and Actuators B: chemical, 100(1):200-204, 2004.

[84] Nima Mohseni Kiasari, Saeid Soltanian, Bobak Gholamkhass, and Peyman Servati. Room temperature ultra-sensitive resistive humidity sensor based on single zinc oxide nanowire. Sensors and Actuators, A: Physical, 182:101105, 2012.

[85] Yongsheng Zhang, Ke Yu, Desheng Jiang, Ziqiang Zhu, Haoran Geng, and Laiqiang Luo. Zinc oxide nanorod and nanowire for humidity sensor. Applied Surface Science, 242(1-2):212-217, 2005.

[86] F. Fang, J. Futter, A. Markwitz, and J. Kennedy. UV and humidity sensing properties of $\mathrm{ZnO}$ nanorods prepared by the arc discharge method. Nanotechnology, 20(24), 2009.

[87] Qi Qi, Tong Zhang, Qingjiang Yu, Rui Wang, Yi Zeng, Li Liu, and Haibin Yang. Properties of humidity sensing $\mathrm{ZnO}$ nanorods-base sensor fabricated by screen-printing. Sensors and Actuators, B: Chemical, 133(2):638-643, 2008. 
[88] Sheng-Po Chang, Shoou-Jinn Chang, Chien-Yuan Lu, Meng-Ju Li, ChengLiang Hsu, Yu-Zung Chiou, Ting-Jen Hsueh, and I-Cherng Chen. A zno nanowire-based humidity sensor. Superlattices and Microstructures, 47(6):772-778, 2010.

[89] Xu Jiaqiang, Chen Yuping, Chen Daoyong, and Shen Jianian. Hydrothermal synthesis and gas sensing characters of $\mathrm{ZnO}$ nanorods. Sensors and Actuators, B: Chemical, 113(1):526-531, 2006.

[90] Xu Jiaqiang, Chen Yuping, Li Yadong, and Shen Jianian. Gas sensing properties of $\mathrm{ZnO}$ nanorods prepared by hydrothermal method. Journal of $\mathrm{Ma}$ terials Science, 40(11):2919-2921, 2005.

[91] Liwei Wang, Yanfei Kang, Xianghong Liu, Shoumin Zhang, Weiping Huang, and Shurong Wang. ZnO nanorod gas sensor for ethanol detection. Sensors and Actuators, B: Chemical, 162(1):237-243, 2012.

[92] J X Wang, X W Sun, Y Yang, H Huang, Y C Lee, O K Tan, and L Vayssieres. Hydrothermally grown oriented $\mathrm{ZnO}$ nanorod arrays for gas sensing applications. Nanotechnology, 17(19):4995-4998, 2006.

[93] Ting-Jen Hsueh, Cheng-Liang Hsu, Shoou-Jinn Chang, and I-Cherng Chen. Laterally grown zno nanowire ethanol gas sensors. Sensors and Actuators B: Chemical, 126(2):473-477, 2007.

[94] Q. Wan, Q. H. Li, Y. J. Chen, T. H. Wang, X. L. He, J. P. Li, and C. L. Lin. Fabrication and ethanol sensing characteristics of $\mathrm{ZnO}$ nanowire gas sensors. Applied Physics Letters, 84(18):3654-3656, 2004.

[95] N Hongsith, C Viriyaworasakul, P Mangkorntong, N Mangkorntong, and $\mathrm{S}$ Choopun. Ethanol sensor based on zno and au-doped zno nanowires. Ceramics International, 34(4):823-826, 2008.

[96] Chandra Sekhar Rout, S Hari Krishna, SRC Vivekchand, A Govindaraj, and CNR Rao. Hydrogen and ethanol sensors based on zno nanorods, nanowires and nanotubes. Chemical Physics Letters, 418(4):586-590, 2006.

[97] Zhi-Peng Sun, Lang Liu, Li Zhang, and Dian-Zeng Jia. Rapid synthesis of $\mathrm{ZnO}$ nano-rods by one-step, room-temperature, solid-state reaction and their gas-sensing properties. Nanotechnology, 17(9):2266-2270, 2006.

[98] Zhiyong Fan and Jia G Lu. Chemical sensing with zno nanowire field-effect transistor. IEEE transactions on nanotechnology, 5(4):393-396, 2006.

[99] Cesare Soci, Arthur Zhang, Bin Xiang, Shadi A Dayeh, DPR Aplin, Jung Park, XY Bao, Yu-Hwa Lo, and Deli Wang. Zno nanowire uv photodetectors with high internal gain. Nano letters, 7(4):1003-1009, 2007. 
[100] Conor P Burke-Govey, Uli Castanet, Harry Warring, A Nau, Ben J Ruck, Jerome Majimel, and Natalie OV Plank. Realizing field-dependent conduction in zno nanowires without annealing. Nanotechnology, 28(12):124003, 2017.

[101] Jung Inn Sohn, Su Seok Choi, Stephen M. Morris, James S. Bendall, Harry J. Coles, Woong Ki Hong, Gunho Jo, Takhee Lee, and Mark E. Welland. Novel nonvolatile memory with multibit storage based on a $\mathrm{ZnO}$ nanowire transistor. Nano Letters, 10(11):4316-4320, 2010.

[102] Woong-Ki Hong, Bong-Joong Kim, Tae-Wook Kim, Gunho Jo, Sunghoon Song, Soon-Shin Kwon, Ahnsook Yoon, Eric A. Stach, and Takhee Lee. Electrical properties of $\mathrm{ZnO}$ nanowire field effect transistors by surface passivation. Colloids and Surfaces A: Physicochemical and Engineering Aspects, 313314(February):378-382, 2008.

[103] Jin Suk Kim, Won Il Park, Chul-Ho Lee, and Gyu-Chul Yi. ZnO nanorod biosensor for highly sensitive detection of specific protein binding. J. Korean Phys. Soc, 49(4):1635-1639, 2006.

[104] Jung Inn Sohn, Woong-Ki Hong, Mi Jung Lee, Takhee Lee, Henning Sirringhaus, Dae Joon Kang, and Mark E Welland. The influence of surface chemical dynamics on electrical and optical properties of $\mathrm{ZnO}$ nanowire field effect transistors. Nanotechnology, 20(50):505202, 2009.

[105] Dunwei Wang, Ying Lan Chang, Qian Wang, Jien Cao, Damon B. Farmer, Roy G. Gordon, and Hongjie Dai. Surface chemistry and electrical properties of germanium nanowires. Journal of the American Chemical Society, 126(37):11602-11611, 2004.

[106] Shadi A. Dayeh, Cesare Soci, Paul K L Yu, Edward T. Yu, and Deli Wang. Influence of surface states on the extraction of transport parameters from InAs nanowire field effect transistors. Applied Physics Letters, 90(16):2-5, 2007.

[107] H. Y. Li, O. Wunnicke, M. T. Borgström, W. G G Immink, M. H M Van Weert, M. A. Verheijen, and E. P A M Bakkers. Remote p-doping of InAs nanowires. Nano Letters, 7(5):1144-1148, 2007.

[108] Wenfeng Zhang, Jiansheng Jie, Linbao Luo, Guodong Yuan, Zhubing He, Zhiqiang Yao, Zhenhua Chen, Chun-Sing Lee, Wenjun Zhang, and ShuitTong Lee. Hysteresis in in 2 o 3: Zn nanowire field-effect transistor and its application as a nonvolatile memory device. Applied Physics Letters, 93(18):183111, 2008.

[109] Dattatray J. Late, Bin Liu, H. S S Ramakrishna Matte, Vinayak P. Dravid, and C. N R Rao. Hysteresis in single-layer MoS 2 field effect transistors. ACS Nano, 6(6):5635-5641, 2012. 
[110] Woong Kim, Ali Javey, Ophir Vermesh, Qian Wang, Yiming Li, and Hongjie Dai. Hysteresis caused by water molecules in carbon nanotube field-effect transistors. Nano Letters, 3(2):193-198, 2003.

[111] Ao Guo, Yunyi Fu, Chuan Wang, Lunhui Guan, Jia Liu, Zujin Shi, Zhennan $\mathrm{Gu}, \mathrm{Ru}$ Huang, and Xing Zhang. Two-bit memory devices based on singlewall carbon nanotubes: Demonstration and mechanism. Nanotechnology, 18(12), 2007.

[112] C Tuerk and L Gold. Systematic evolution of ligands by exponential enrichment: RNA ligands to bacteriophage T4 DNA polymerase. Science (New York, N.Y.), 249(4968):505-510, 1990.

[113] Andrew D. Ellington and Jack W. Szostak. In vitro selection of RNA molecules that bind specific ligands. Nature, 346(6287):818-822, 1990.

[114] Andrew D. Ellington and Jack W. Szostak. Selection in vitro of singlestranded DNA molecules that fold into specific ligand-binding structures. Nature, 355(6363):850-852, 1992.

[115] James D Watson, Francis HC Crick, et al. Molecular structure of nucleic acids. Nature, 171(4356):737-738, 1953.

[116] Abd-Elgawad Radi and Ciara K. O'Sullivan. Aptamer conformational switch as sensitive electrochemical biosensor for potassium ion recognition. Chemical Communications, (32):3432, 2006.

[117] * Milan N. Stojanovic, Paloma de Prada, , and Donald W. Landry*. Aptamer-Based Folding Fluorescent Sensor for Cocaine. 4(11):4928-4931, 2001.

[118] Han Yue Zheng, Omar A Alsager, Bicheng Zhu, Jadranka Travas-Sejdic, Justin M Hodgkiss, and Natalie OV Plank. Electrostatic gating in carbon nanotube aptasensors. Nanoscale, 8(28):13659-13668, 2016.

[119] Brian R Baker, Rebecca Y Lai, McCall S Wood, Elaine H Doctor, Alan J Heeger, and Kevin W Plaxco. An electronic, aptamer-based small-molecule sensor for the rapid, label-free detection of cocaine in adulterated samples and biological fluids. Journal of the American Chemical Society, 128(10):31383139, 2006.

[120] Joshua A. Hagen, Sang N. Kim, Burhan Bayraktaroglu, Nancy KelleyLoughnane, Rajesh R. Naik, and Morley O. Stone. DNA aptamer functionalized zinc oxide field effect transistors for liquid state selective sensing of small molecules. 7759:775912, 2010.

[121] Joshua A. Hagen, Sang N. Kim, Burhan Bayraktaroglu, Kevin Leedy, Jorge L. Chávez, Nancy Kelley-Loughnane, Rajesh R. Naik, and Morley O. Stone. Biofunctionalized zinc oxide field effect transistors for selective sensing of riboflavin with current modulation. Sensors, 11(7):6645-6655, 2011. 
[122] Joshua a. Hagen, Sang Nyon Kim, Nancy Kelley-Loughnane, Rajesh R. Naik, and Morley O. Stone. Selective vapor phase sensing of small molecules using biofunctionalized field effect transistors. Proceedings of SPIE, 8018:80180B-80180B-7, 2011.

[123] M. Singh, M. Y. Mulla, K. Manoli, M. Magliulo, N. Ditaranto, N. Cioffi, G. Palazzo, L. Torsi, M. V. Santacroce, C. Di'Franco, and G. Scamarcio. Biofunctionalization of $\mathrm{ZnO}$ water gated thin-film transistors. Proceedings 2015 6th IEEE International Workshop on Advances in Sensors and Interfaces, IWASI 2015, pages 261-265, 2015.

[124] Raphael Niepelt, Ulrich C Schröder, Jana Sommerfeld, Irma Slowik, Bettina Rudolph, Robert Möller, Barbara Seise, Andrea Csaki, Wolfgang Fritzsche, and Carsten Ronning. Biofunctionalization of zinc oxide nanowires for DNA sensory applications. Nanoscale Research Letters, 6(1):511, 2011.

[125] Ahmi Choi, Kyoungwon Kim, Hyo Il Jung, and Sang Yeol Lee. ZnO nanowire biosensors for detection of biomolecular interactions in enhancement mode. Sensors and Actuators, B: Chemical, 148(2):577-582, 2010.

[126] Sajal Shrivastava, Nguyen Minh Triet, Young Min Son, Won Il Lee, and Nae Eung Lee. Seesawed fluorescence nano-aptasensor based on highly vertical $\mathrm{ZnO}$ nanorods and three-dimensional quantitative fluorescence imaging for enhanced detection accuracy of ATP. Biosensors and Bioelectronics, 90(September 2016):450-458, 2017.

[127] K Seshan. Handbook of Thin-Film Deposition Processes and Techniques, Second Edition. William Andrew Publising, 2000.

[128] J Liu, B Cai, J Zhu, G Ding, X Zhao, C Yang, and D Chen. Process research of high aspect ratio microstructure using su-8 resist. Microsystem Technologies, 10(4):265-268, 2004.

[129] H. W. Ra, R. Khan, J. T. Kim, B. R. Kang, K. H. Bai, and Y. H. Im. Effects of surface modification of the individual $\mathrm{ZnO}$ nanowire with oxygen plasma treatment. Materials Letters, 63(28):2516-2519, 2009.

[130] Hyun-Wook Ra and Yeon-Ho Im. Effect of chemically reactive species on properties of $\mathrm{ZnO}$ nanowires exposed to oxygen and hydrogen plasma. Nanotechnology, 19(48):485710, 2008.

[131] Sandeep Kumar Vashist, Edmond Lam, Sabahudin Hrapovic, Keith B. Male, and John H T Luong. Immobilization of antibodies and enzymes on 3-aminopropyltriethoxysilane-functionalized bioanalytical platforms for biosensors and diagnostics. Chemical Reviews, 114(21):1108311130, 2014.

[132] Gyu-Chul Yi, Chunrui Wang, and Won Il Park. ZnO nanorods: synthesis, characterization and applications. Semiconductor Science and Technology, 20(4):S22-S34, 2005. 
[133] Myoung Soo Kim, Jang Hwan Han, Da Hyeok Lee, Beom Hoan O, Seung Gol Lee, El Hang Lee, and Se Geun Park. Laterally grown ZnO nanorod arrays on an obliquely deposited seed layer and its UV photocurrent response. Microelectronic Engineering, 97(3):130-133, 2012.

[134] Yong Qin, Rusen Yang, and Zhong Lin Wang. Growth of Horizonatal ZnO Nanowire Arrays on Any Substrate Growth of Horizonatal ZnO Nanowire Arrays on Any Substrate. 112(November):18734-18736, 2008.

[135] Guang Zhu, Rusen Yang, Sihong Wang, and Zhong Lin Wang. Flexible high-output nanogenerator based on lateral $\mathrm{ZnO}$ nanowire array. Nano Letters, 10(8):3151-3155, 2010.

[136] AN Nigam, RP Tripathi, and K Dhoot. The effect of phosphoric acid on rust studied by mössbauer spectroscopy. Corrosion Science, 30(8-9):799-803, 1990.

[137] Peter J. Hotchkiss, Michał Malicki, Anthony J. Giordano, Neal R. Armstrong, and Seth R. Marder. Characterization of phosphonic acid binding to zinc oxide. Journal of Materials Chemistry, 21(9):3107, 2011.

[138] GH Aylward and TJV Findlay. SI Chemical Data, Sixth Edition. Wiley, 2008.

[139] Vivek Pachauri, Alexis Vlandas, Klaus Kern, and Kannan Balasubramanian. Site-specific self-assembled liquid-gated $\mathrm{ZnO}$ nanowire transistors for sensing applications. Small, 6(4):589-594, 2010.

[140] RM Robson, Darrel E Goll, and MJ Temple. Determination of proteins in tris buffer by the biuret reaction. Analytical biochemistry, 24(2):339-341, 1968.

[141] Tris Buffer. Hampton Research, retreived 30.01.2018 from https://hamptonresearch.com/.

[142] Hongtao Yuan, Hidekazu Shimotani, Atsushi Tsukazaki, Akira Ohtomo, Masashi Kawasaki, and Yoshihiro Iwasa. Hydrogenation-induced surface polarity recognition and proton memory behavior at protic-ionicliquid/oxide electric-double-layer interfaces. Journal of the American Chemical Society, 132(19):6672-6678, 2010.

[143] Bernd Meyer, Dominik Marx, Olga Dulub, Ulrike Diebold, Martin Kunat, Deler Langenberg, and Christof Wöll. Partial dissociation of water leads to stable superstructures on the surface of zinc oxide. Angewandte Chemie International Edition, 43(48):6642-6645, 2004.

[144] Olga Dulub, Bernd Meyer, and Ulrike Diebold. Observation of the dynamical change in a water monolayer adsorbed on a $\mathrm{ZnO}$ surface. Physical Review Letters, 95(13):1-4, 2005. 
[145] Jun Zhou, Ningsheng $\mathrm{Xu}$, and Zhong L. Wang. Dissolving behavior and stability of $\mathrm{ZnO}$ wires in biofluids: A study on biodegradability and biocompatibility of $\mathrm{ZnO}$ nanostructures. Advanced Materials, 18(18):2432-2435, 2006.

[146] Karin H. Müller, Jaideep Kulkarni, Michael Motskin, Angela Goode, Peter Winship, Jeremy N. Skepper, Mary P. Ryan, and Alexandra E. Porter. PHdependent toxicity of high aspect ratio $\mathrm{ZnO}$ nanowires in macrophages due to intracellular dissolution. ACS Nano, 4(11):6767-6779, 2010.

[147] Chun Cheng, Renlong Xin, Yang Leng, Dapeng Yu, and Ning Wang. Chemical stability of zno nanostructures in simulated physiological environments and its application in determining polar directions. Inorganic chemistry, 47(17):7868-7873, 2008.

[148] John A. Dean. Lange's Handbook of Chemistry, Fifteenth Edition. McGraw-Hill Inc, 1999.

[149] Meteorological Service of New Zealand Ltd. Surface Pressure Charts, 2018.

[150] Camelia Florica, Andreea Costas, Andrei Kuncser, Nicoleta Preda, and Ionut Enculescu. High performance FETs based on $\mathrm{ZnO}$ nanowires synthesized by low cost methods. Nanotechnology, 27(47):475303, 2016.

[151] R. Kochetov, T. Andritsch, U. Lafont, P. H F Morshuis, and J. J. Smit. Effects of inorganic nanofillers and combinations of them on the complex permittivity of epoxy-based composites. Conference Record of IEEE International Symposium on Electrical Insulation, pages 1-5, 2010.

[152] H. Frenzel, A. Lajn, M. Brandt, H. Von Wenckstern, G. Biehne, H. Hochmuth, M. Lorenz, and M. Grundmann. ZnO metal-semiconductor field-effect transistors with Ag-Schottky gates. Applied Physics Letters, 92(19):2006-2009, 2008.

[153] Heiko Frenzel, Alexander Lajn, Holger Von Wenckstern, Michael Lorenz, Friedrich Schein, Zhipeng Zhang, and Marius Grundmann. Recent progress on $\mathrm{ZnO}$-based metal-semiconductor field-effect transistors and their application in transparent integrated circuits. Advanced Materials, 22(47):5332-5349, 2010.

[154] Jeseung Oh, Young Wook Chang, Hyung Joon Kim, Seunghwan Yoo, Dong Jun Kim, Seongil Im, Young June Park, Donghyun Kim, and Kyung Hwa Yoo. Carbon nanotube-based dual-mode biosensor for electrical and surface plasmon resonance measurements. Nano Letters, 10(8):27552760, 2010.

[155] Jeseung Oh, Gu Yoo, Young Wook Chang, Hyung Joon Kim, Joachim Jose, Eosu Kim, Jae Chul Pyun, and Kyung Hwa Yoo. A carbon nanotube metal semiconductor field effect transistor-based biosensor for detection 
of amyloid-beta in human serum. Biosensors and Bioelectronics, 50:345-350, 2013. 